\title{
Well-Posedness and Stability Results for Some Periodic Muskat Problems
}

Bogdan-Vasile Matioc

\author{
Communicated by M. Hieber
}

\begin{abstract}
We study the two-dimensional Muskat problem in a horizontally periodic setting and for fluids with arbitrary densities and viscosities. We show that in the presence of surface tension effects the Muskat problem is a quasilinear parabolic problem which is well-posed in the Sobolev space $H^{r}(\mathbb{S})$ for each $r \in(2,3)$. When neglecting surface tension effects, the Muskat problem is a fully nonlinear evolution equation and of parabolic type in the regime where the RayleighTaylor condition is satisfied. We then establish the well-posedness of the Muskat problem in the open subset of $H^{2}(\mathbb{S})$ defined by the Rayleigh-Taylor condition. Besides, we identify all equilibrium solutions and study the stability properties of trivial and of small finger-shaped equilibria. Also other qualitative properties of solutions such as parabolic smoothing, blow-up behavior, and criteria for global existence are outlined.
\end{abstract}

Mathematics Subject Classification. 35B35, 35B65, 35K55, 35Q35, 42B20.

Keywords. Muskat problem, Singular integral, Well-posedness, Parabolic smoothing, Stability.

\section{Contents}

1. Introduction and the Main Results 1

2. The Equations of Motion and the Equivalence of the Formulations 6

3. The Double Layer Potential and Its Adjoint 9

4. The Muskat Problem with Surface Tension Effects 16

5. The Muskat Problem Without Surface Tension Effects 27

6. Stability Analysis $\quad 35$

Appendix A. Some Technical Results $\quad 39$

References $\quad 43$

\section{Introduction and the Main Results}

In this paper we study the coupled system of equations

$$
\left\{\begin{aligned}
\partial_{t} f(t, x) & =\frac{1}{4 \pi} \mathrm{PV} \int_{-\pi}^{\pi} \frac{f^{\prime}(t, x)\left(1+t_{[s]}^{2}\right)\left(T_{[x, s]} f(t)\right)+t_{[s]}\left[1-\left(T_{[x, s]} f(t)\right)^{2}\right]}{t_{[s]}^{2}+\left(T_{[x, s]} f(t)\right)^{2}} \bar{\omega}(t, x-s) \mathrm{d} s, \\
\bar{\omega}(t, x)= & \frac{2 k}{\mu_{-}+\mu_{+}}(\sigma \kappa(f(t))-\Theta f(t))^{\prime}(x) \\
& -\frac{a_{\mu}}{2 \pi} \mathrm{PV} \int_{-\pi}^{\pi} \frac{f^{\prime}(t, x) t_{[s]}\left[1-\left(T_{[x, s]} f(t)\right)^{2}\right]-\left(1+t_{[s]}^{2}\right) T_{[x, s]} f(t)}{t_{[s]}^{2}+\left(T_{[x, s]} f(t)\right)^{2}} \bar{\omega}(t, x-s) \mathrm{d} s
\end{aligned}\right.
$$


for $t>0^{1}$ and $x \in \mathbb{R}$, which is supplemented by the initial condition

$$
f(0)=f_{0} .
$$

The evolution problem (1.1) describes the motion of the boundary $[y=f(t, x)+t V]$ separating two immiscible fluid layers with unbounded heights located in a homogeneous porous medium with permeability $k \in(0, \infty)$ or in a vertical/horizontal Hele-Shaw cell. It is assumed that the fluid system moves with constant velocity $(0, V), V \in \mathbb{R}$, that the motion is periodic with respect to the horizontal variable $x$ (with period $2 \pi$ ), and that the fluid velocities are asymptotically equal to $(0, V)$ far away from the interface. The unknowns of the evolution problem (1.1) are the functions $(f, \bar{\omega})=(f, \bar{\omega})(t, x)$. We denote by $\mathbb{S}:=\mathbb{R} / 2 \pi \mathbb{Z}$ the unit circle, functions that depend on $x \in \mathbb{S}$ being $2 \pi$-periodic with respect to the real variable $x$. To be concise, we have set

$$
\delta_{[x, s]} f:=f(x)-f(x-s), \quad T_{[x, s]} f=\tan h\left(\frac{\delta_{[x, s]} f}{2}\right), \quad t_{[s]}=\tan \left(\frac{s}{2}\right),
$$

and $(\cdot)^{\prime}$ denotes the spatial derivative $\partial_{x}$. We further denote by $g$ the Earth's gravity, $\sigma \in[0, \infty)$ is the surface tension coefficient, $\kappa(f(t))$ is the curvature of the free boundary $[y=f(t, x)+t V]$, while $\mu_{ \pm}$and $\rho_{ \pm}$are the viscosity and the density, respectively, of the fluid \pm which occupies the unbounded periodic strip

$$
\Omega_{ \pm}^{V}(t):=\left\{(x, y) \in \mathbb{R}^{2}: \pm(f(t, x)+t V-y)<0\right\} .
$$

Moreover, the real constant $\Theta$ and the Atwood number $a_{\mu}$ that appear in (1.1a) $)_{2}$ are defined by

$$
\Theta:=g\left(\rho_{-}-\rho_{+}\right)+\frac{\mu_{-}-\mu_{+}}{k} V, \quad a_{\mu}:=\frac{\mu_{-}-\mu_{+}}{\mu_{-}+\mu_{+}} .
$$

The integrals in (1.1a) are singular at $s=0$ and PV denotes the Cauchy principle value. In this paper we consider a general setting where

$$
\mu_{-}-\mu_{+}, \rho_{-}-\rho_{+} \in \mathbb{R} .
$$

The observation that $\left|a_{\mu}\right|<1$ is crucial for our analysis. This property enables us to prove, for suitable $f(t)$, that the Eq. (1.1a) $)_{2}$ has a unique solution $\bar{\omega}(t)$ (which depends in an intricate way on $f(t)$, see Sects. 4 and 5). Therefore we shall only refer to $f$ as being the solution to (1.1).

The Muskat problem, in the classical formulation (2.1), dates back to M. Muskat's paper [52] from 1934. However, many of the mathematical studies on this topic are quite recent and they cover various physical scenarios and mathematical aspects related to the original model proposed in [52], cf. [6,7,9,11$18,21-25,27,30,32,36,38-43,48,49,49,53,54,58,60-62]$ (see also $[55,56]$ for some recent research on the compressible analogue of the Muskat problem, the so-called Verigin problem).

Below we discuss only the literature pertaining to (1.1) and its nonperiodic counterpart. In the presence of surface tension effects, that is for $\sigma>0,(1.1)$ has been studied previously only in [7] where the author proved well-posedness of the problem in $H^{r}$ (with $r \geq 6$ ) in the more general setting of interfaces which are parameterized by curves, and the zero surface tension limit of the problem has been also considered there. The nonperiodic counterpart to (1.1) has been investigated in [48] where it was shown that the problem is well-posed in $H^{r}(\mathbb{S})$ for each $r \in(2,3)$ by exploiting the fact that the problem is quasilinear parabolic together with the abstract theory outlined in $[4,5]$ for such problems. Additionally, it was shown in [48] that the problem exhibits the effect of parabolic smoothing and criteria for global existence of solutions were found. We showed herein that the results in the nonperiodic framework [48] hold also for (1.1). Besides, this paper provides the first full picture of the set of equilibrium solutions to (1.1) — which are described by either flat of finger-shaped interfaces - and the stability properties of the flat equilibria and of small finger-shaped equilibria are studied in the natural phase space $H^{r}(\mathbb{S})$. For the latter purpose we use a quasilinear principle of linearized stability established recently in [50].

\footnotetext{
${ }^{1}$ When $\sigma=0$ we require that the Eq. (1.1a) are satisfied also at $t=0$.
} 
The first main result of this paper is the following theorem establishing the well-posedness of the Muskat problem with surface tension in the setting of classical solutions and for general initial data together with other qualitative properties of the solutions.

Theorem 1.1. Let $\sigma>0$ and $r \in(2,3)$ be given. Then, the following hold:

(i) (Well-posedness in $H^{r}(\mathbb{S})$ ) The problem (1.1) possesses for each $f_{0} \in H^{r}(\mathbb{S})$ a unique maximal solution

$$
f:=f\left(\cdot ; f_{0}\right) \in \mathrm{C}\left(\left[0, T_{+}\left(f_{0}\right)\right), H^{r}(\mathbb{S})\right) \cap \mathrm{C}\left(\left(0, T_{+}\left(f_{0}\right)\right), H^{3}(\mathbb{S})\right) \cap \mathrm{C}^{1}\left(\left(0, T_{+}\left(f_{0}\right)\right), L_{2}(\mathbb{S})\right),
$$

with $T_{+}\left(f_{0}\right) \in(0, \infty]$, and $\left[\left(t, f_{0}\right) \mapsto f\left(t ; f_{0}\right)\right]$ defines a semiflow on $H^{r}(\mathbb{S})$.

(ii) (Global existence/blow-up criterion) If

$$
\sup _{\left[0, T_{+}\left(f_{0}\right)\right) \cap[0, T]}\left\|f\left(t ; f_{0}\right)\right\|_{H^{r}}<\infty \quad \text { for all } T>0,
$$

then $T_{+}\left(f_{0}\right)=\infty$.

(iii) (Parabolic smoothing) The mapping $[(t, x) \mapsto f(t, x)]:\left(0, T_{+}\left(f_{0}\right)\right) \times \mathbb{R} \rightarrow \mathbb{R}$ is real-analytic. In particular, $f(t)$ is a real-analytic function for all $t \in\left(0, T_{+}\left(f_{0}\right)\right)$.

Remark 1.2. (i) Despite that we deal with a third order problem in the setting of classical solutions, the curvature of the initial data in Theorem 1.1 may be unbounded and/or discontinuous. Moreover, it becomes instantaneously real-analytic under the flow.

(ii) Solutions which are not global have, in view of Theorem 1.1, the property that

$$
\sup _{\left[0, T_{+}\left(f_{0}\right)\right)}\|f(t)\|_{H^{s}}=\infty \quad \text { for each } s \in(2,3) .
$$

Concerning the stability of equilibria, we also have to differentiate between the cases $\sigma=0$ and $\sigma>0$. Before doing this we point out two features that are common for both cases. Firstly, the integral mean of the solutions to (1.1) (found in Theorems 1.1 or 1.5 below) is constant with respect to time, see Sect. 6 . Secondly, (1.1) has the following invariance property: If $f$ is a solution to (1.1), then the translation

$$
f_{a, c}(t, x):=f(t, x-a)+c, \quad a, c \in \mathbb{R},
$$

is also a solution to (1.1). For these two reasons, we shall only address the stability issue for equilibria to (1.1) which have zero integral mean and under perturbations with zero integral mean. However, because of the invariance property (1.2), our stability results can be transferred also to other equilibria, see Remark 1.4.

To set the stage, let

$$
\widehat{H}^{r}(\mathbb{S}):=\left\{h \in H^{r}(\mathbb{S}):\langle h\rangle:=\frac{1}{2 \pi} \int_{-\pi}^{\pi} h \mathrm{~d} x=0\right\}, \quad r \geq 0 .
$$

In Theorem 1.3 below we describe the stability properties of some of the equilibria to (1.1) when $\sigma>0$. In this case the equilibrium solutions to (1.1) are either constant functions or finger-shaped as in Fig. 1. The finger-shaped equilibria exist only in the regime where $\Theta<0$, that is when either the fluid located below has a larger density or when the less viscous fluid advances into the region occupied by the other one with sufficiently high speed $|V|$. Furthermore, these equilibria form global bifurcation branches (see Sect. 6 for the complete picture of the set of equilibria).

Theorem 1.3. Let $\sigma>0$ and $r \in(2,3)$ be given. The following hold:

(i) If $\Theta+\sigma>0$, then $f=0$ is exponentially stable. More precisely, given

$$
\omega \in\left(0, k(\sigma+\Theta) /\left(\mu_{-}+\mu_{+}\right)\right),
$$

there exist constants $\delta>0$ and $M>0$, with the property that if $f_{0} \in \widehat{H}^{r}(\mathbb{S})$ satisfies $\left\|f_{0}\right\|_{H^{r}} \leq \delta$, the solution to (1.1) exists globally and

$$
\left\|f\left(t ; f_{0}\right)\right\|_{H^{r}} \leq M e^{-\omega t}\left\|f_{0}\right\|_{H^{r}} \quad \text { for all } t \geq 0 .
$$


(ii) If $\Theta+\sigma<0$, then $f=0$ is unstable. More precisely, there exists $R>0$ and a sequence $\left(f_{0, n}\right) \subset \widehat{H}^{r}(\mathbb{S})$ of initial data such that:

- $f_{0, n} \rightarrow 0$ in $\widehat{H}^{r}(\mathbb{S})$;

- There exists $t_{n} \in\left(0, T_{+}\left(f_{0, n}\right)\right)$ with $\left\|f\left(t_{n} ; f_{0, n}\right)\right\|_{H^{r}}=R$.

(iii) (Instability of small finger shaped equilibria) Given $1 \leq \ell \in \mathbb{N}$, there exists a real-analytic bifurcation curve $\left(\lambda_{\ell}, f_{\ell}\right):\left(-\varepsilon_{\ell}, \varepsilon_{\ell}\right) \rightarrow(0, \infty) \times \widehat{H}^{3}(\mathbb{S}), \varepsilon_{\ell}>0$, with

$$
\left\{\begin{array}{l}
\lambda_{\ell}(s)=\ell^{2}-\frac{3 \ell^{4}}{8} s^{2}+O\left(s^{4}\right) \quad \text { in } \mathbb{R}, \\
f_{\ell}(s)=s \cos (\ell x)+O\left(s^{2}\right) \quad \text { in } \widehat{H}^{3}(\mathbb{S})
\end{array} \quad \text { for } s \rightarrow 0,\right.
$$

such that $f_{\ell}(s)$ is an even equilibrium to (1.1) if $\Theta=-\sigma \lambda_{\ell}(s)$. The finger-shaped equilibrium $f_{\ell}(s)$, $0<|s|<\varepsilon_{\ell}$, is unstable if $\varepsilon_{\ell}$ is sufficiently small in the sense there exists $R>0$ and a sequence $\left(f_{0, n}\right) \subset \widehat{H}^{r}(\mathbb{S})$ such that:

- $f_{0, n} \rightarrow f_{\ell}(s)$ in $\widehat{H}^{r}(\mathbb{S})$;

- There exists $t_{n} \in\left(0, T_{+}\left(f_{0, n}\right)\right)$ with $\left\|f\left(t_{n} ; f_{0, n}\right)-f_{\ell}(s)\right\|_{H^{r}}=R$.

With respect to Theorem 1.3 we add the following remarks (Remark $1.4(i)$ remains valid for Theorem 1.6 below as well).

Remark 1.4. (i) If $f$ is an even equilibrium to (1.1), the translation $f(\cdot-a)+c, a, c \in \mathbb{R}$, is also an equilibrium solution. In fact, all equilibria can be obtained in this way (see Sect. 6). The invariance property (1.2) shows that $f$ and $f(\cdot-a)+c$ have the same stability properties.

(ii) It is shown in Theorem 6.1 that the local curves $\left(\lambda_{\ell}, f_{\ell}\right)$ can be continued to global bifurcation branches consisting entirely of equilibrium solutions to (1.1). The stability issue for the large fingershaped equilibria remains an open problem.

When switching to the regime where $\sigma=0$, many aspects in the analysis of the Muskat problem with surface tension have to be reconsidered. A first major difference to the case $\sigma>0$ is due to the fact that the quasilinear character of the problem, which is mainly due to the curvature term, is lost (except for the very special case when $\mu_{-}=\mu_{+}$, cf. [47]), and the problem (1.1) is now fully nonlinear. The second important difference, is that the problem is of parabolic type only when the Rayleigh-Taylor condition holds. The Rayleigh-Taylor condition originates from [59] and is expressed in terms of the pressures $p_{ \pm}$ associated of the fluid \pm as follows

$$
\partial_{\nu} p_{-}<\partial_{\nu} p_{+} \quad \text { on }\left[y=f_{0}(x)\right]
$$

with $\nu$ denoting the unit normal to the curve $\left[y=f_{0}(x)\right]$ pointing towards $\Omega_{+}^{V}(0)$. The first result in this setting is a local existence result in $H^{k}(\mathbb{S})$, with $k \geq 3$, established in [21] in the more general setting of interfaces parametrized by periodic curves (for initial data such that the Rayleigh-Taylor conditions holds). The particular case of fluids with equal densities has been in fact investigated previously in [60] and the authors have shown the existence of global solutions for small data. The methods from [21] have been then generalized in [22] to the three-dimensional case, the analysis leading to a local existence result in $H^{k}$ with $k \geq 4$. More recently in [15] the authors have established global existence and uniqueness of solutions to $(1.1)$ for small data in $H^{2}(\mathbb{S})$ together with some exponential decay estimates in $H^{r}$-norms with $r \in[0,2)$. For the nonperiodic Muskat problem with $\sigma=0$ it is moreover shown in [15] there exist unique local solutions for initial data in $H^{2}(\mathbb{R})$ which are small in the weaker $H^{3 / 2+\varepsilon}$-norm with $\varepsilon \in(0,1)$ arbitrarily small. The latter smallness size condition on the data was dropped in [48] where it is shown that the nonperiodic Muskat possesses for initial data in $H^{2}(\mathbb{R})$ that satisfy the Rayleigh-Taylor condition a unique local solution and that the solution depends continuously on the data. Lastly, we mention the paper [37] where the existence and uniqueness of a weaker notion of solutions is established for the nonperiodic Muskat problem with initial data in critical spaces, together with some algebraic decay of the global solutions. In this paper we first generalize the methods from the nonperiodic setting [48] to prove the well-posedness of (1.1) for general initial data in $H^{2}(\mathbb{S})$ and instantaneous parabolic 
smoothing for solutions which satisfy an additional bound. Before presenting our result, we point out that if $\Theta=0$, then (1.1) has only constant solutions for each $f_{0} \in H^{r}(\mathbb{R})$, with $r>3 / 2$, as Theorem 3.3 shows that in this case $\bar{\omega}=0$ is the only solution to $(1.1 \mathrm{a})_{2}$ that lies in $\widehat{L}_{2}(\mathbb{S})$. When $\Theta \neq 0$, the situation is much more complex. Letting

$$
\mathcal{O}:=\left\{f_{0} \in H^{2}(\mathbb{S}): \partial_{\nu} p_{-}<\partial_{\nu} p_{+} \text {on }\left[y=f_{0}(x)\right]\right\}
$$

denote the set of initial data in $H^{2}(\mathbb{S})$ for which the Rayleigh-Taylor condition holds, it is shown in Sect. 5 that $\mathcal{O}$ is nonempty precisely when $\Theta>0$. This condition on the constants has been identified also in the nonperiodic case. In fact, we prove that if $\Theta>0$, then $\mathcal{O}$ is an open subset of $H^{2}(\mathbb{S})$ which contains all constant functions. Using the abstract fully nonlinear parabolic theory established in $[26,46]$, we prove below that the Muskat problem without surface tension is well-posed in the set $\mathcal{O}$, cf. Theorem 1.5. Physically, in the particular situation when gravity is neglected $\Theta>0$ is equivalent to the fact that the more viscous fluid enters the region occupied by less viscous one, while in the case $V=0$ the condition $\Theta>0$ means that the fluid located below has a larger density.

Theorem 1.5. Let $\sigma=0, \mu_{-} \neq \mu_{+},{ }^{2}$ and assume that $\Theta>0$. Given $f_{0} \in \mathcal{O}$, the problem (1.1) possesses a solution

$$
f \in C([0, T], \mathcal{O}) \cap C^{1}\left([0, T], H^{1}(\mathbb{S})\right) \cap C_{\alpha}^{\alpha}\left((0, T], H^{2}(\mathbb{S})\right)
$$

for some $T>0$ and an arbitrary $\alpha \in(0,1)$. Additionally, the following statements are true:

(i) $f$ is the unique solution to (1.1) belonging to

$$
\bigcup_{\beta \in(0,1)} C([0, T], \mathcal{O}) \cap C^{1}\left([0, T], H^{1}(\mathbb{S})\right) \cap C_{\beta}^{\beta}\left((0, T], H^{2}(\mathbb{S})\right) .
$$

(ii) $f$ may be extended to a maximally defined solution

$$
f\left(\cdot ; f_{0}\right) \in C\left(\left[0, T_{+}\left(f_{0}\right)\right), \mathcal{O}\right) \cap C^{1}\left(\left[0, T_{+}\left(f_{0}\right)\right), H^{1}(\mathbb{S})\right) \cap \bigcap_{\beta \in(0,1)} C_{\beta}^{\beta}\left((0, T], H^{2}(\mathbb{S})\right)
$$

for all $T<T_{+}\left(f_{0}\right)$, where $T_{+}\left(f_{0}\right) \in(0, \infty]$.

(iii) The solution map $\left[\left(t, f_{0}\right) \mapsto f\left(t ; f_{0}\right)\right]$ defines a semiflow on $\mathcal{O}$ which is real-analytic in the open set $\left\{\left(t, f_{0}\right): f_{0} \in \mathcal{O}, 0<t<T_{+}\left(f_{0}\right)\right\}$.

(iv) If $f\left(\cdot ; f_{0}\right):\left[0, T_{+}\left(f_{0}\right)\right) \cap[0, T] \rightarrow \mathcal{O}$ is uniformly continuous for all $T>0$, then either $T_{+}\left(f_{0}\right)=\infty$, or

$$
T_{+}\left(f_{0}\right)<\infty \text { and } \operatorname{dist}\left(f\left(t ; f_{0}\right), \partial \mathcal{O}\right) \rightarrow 0 \text { for } t \rightarrow T_{+}\left(f_{0}\right) .
$$

(v) If $f\left(\cdot ; f_{0}\right) \in B\left((0, T), H^{2+\varepsilon}(\mathbb{S})\right)$ for some $T \in\left(0, T_{+}\left(f_{0}\right)\right)$ and $\varepsilon \in(0,1)$ arbitrary small, then

$$
f \in C^{\omega}((0, T) \times \mathbb{R}, \mathbb{R}) .
$$

The assertions of Theorem 1.5 are weaker compared to that of Theorem 1.1. For example the uniqueness claim at $(i)$ is established in the setting of strict solutions (in the sense of [46, Chapter 8]) which belong additionally to some singular Hölder space

$$
C_{\beta}^{\beta}\left((0, T], H^{2}(\mathbb{S})\right):=\left\{u \in B\left((0, T], H^{2}(\mathbb{S})\right): \sup _{s \neq t} \frac{\left\|t^{\beta} u(t)-s^{\beta} u(s)\right\|_{H^{2}}}{|t-s|^{\beta}}<\infty\right\}
$$

with $\beta \in(0,1)$. This drawback results from the fact that in the absence of surface tension effects we deal with a fully nonlinear (and nonlocal) problem. We also point out that the parabolic smoothing property established at $(v)$ holds only for solutions $f\left(\cdot ; f_{0}\right) \in B\left((0, T), H^{2+\varepsilon}(\mathbb{S})\right)$ for some $\varepsilon>0$. This additional boundedness condition is needed because the space-time translation

$$
[u \mapsto[(t, x) \mapsto u(a t, x+b t)]]
$$

\footnotetext{
${ }^{2}$ Theorem 1.5 is still valid if $\mu_{-}=\mu_{+}$, however its claims can be improved, cf. [47, Theorem 1.1], as the problem (1.1) is under this restriction of quasilinear type.
} 
does not define for $a, b>0$ a bounded operator between these singular Hölder spaces. This property hiders us to use the parameter trick from the proof of Theorem 1.1 to establish parabolic smoothing for all solutions in Theorem 1.5. However, the boundedness hypothesis imposed at $(v)$ is satisfied if $f_{0} \in \mathcal{O} \cap H^{3}(\mathbb{S})$ because the statements $(i)-(i v)$ in Theorem 1.5 remain true when replacing $H^{k}(\mathbb{S})$ by $H^{k+1}(\mathbb{S})$ for $k \in\{1,2\}$ (possibly with a smaller maximal existence time).

Finally, we point out that in the case when $\sigma=0$ the equilibrium solutions to (1.1) are the constant functions. Theorem 1.6 states that the zero solution to (1.1) (and therewith all other equilibria) is exponentially stable under perturbations with zero integral mean.

Theorem 1.6 (Exponential stability). Let $\sigma=0$ and $\Theta>0$. Then, given $\omega \in\left(0, k \Theta /\left(\mu_{-}+\mu_{+}\right)\right)$, there exist constants $\delta>0$ and $M>0$, with the property that if $f_{0} \in \widehat{H}^{2}(\mathbb{S})$ satisfies $\left\|f_{0}\right\|_{H^{2}} \leq \delta$, then $T_{+}\left(f_{0}\right)=\infty$ and $d^{3}$

$$
\|f(t)\|_{H^{2}}+\|\dot{f}(t)\|_{H^{1}} \leq M e^{-\omega t}\left\|f_{0}\right\|_{H^{2}} \quad \text { for all } t \geq 0 .
$$

Before proceeding with our analysis we emphasize that the periodic case considered herein is more involved that the "canonical" nonperiodic Muskat problem because abstract results from harmonic analysis, cf. [51, Theorem 1], which directly apply to the nonperiodic case (in order to establish useful mapping properties and commutator estimates) have no correspondence in the set of periodic functions. However, we derive in Appendix A, by using the results from the nonperiodic case [48,49], the boundedness of certain multilinear singular integral operators which can be directly applied in the proofs. A further drawback of the Eq. (1.1a) is that some of the integral terms are of lower order and some of the arguments are therefore lengthy. Finally, we point out that the stability issue remains an open question for the nonperiodic counterpart of (1.1).

\section{The Equations of Motion and the Equivalence of the Formulations}

In this section we present the classical formulation of the Muskat problem (see (2.1) below) introduced in [52] and prove that this formulation is equivalent to the contour integral formulation (1.1) in a quite general setting, cf. Proposition 2.3.

We first introduce the equations of motion. In the fluid layers the dynamic is governed by the equations

$$
\left\{\begin{array}{l}
\operatorname{div} v_{ \pm}(t)=0, \\
v_{ \pm}(t)=-\frac{k}{\mu_{ \pm}}\left(\nabla p_{ \pm}(t)+\left(0, \rho_{ \pm} g\right)\right)
\end{array} \quad \text { in } \Omega_{ \pm}^{V}(t),\right.
$$

where $v_{ \pm}(t):=\left(v_{ \pm}^{1}(t), v_{ \pm}^{2}(t)\right)$ denotes the velocity field of the fluid \pm . While $(2.1 \mathrm{a})_{1}$ is the incompressibility condition, the Eq. (2.1a) $)_{2}$ is known as Darcy's law. This linear relation is frequently used for flows which are laminar, cf. [10]. These equations are supplemented by the following boundary conditions at the free interface

$$
\left\{\begin{array}{l}
p_{+}(t)-p_{-}(t)=\sigma \kappa(f(t)), \\
\left\langle v_{+}(t) \mid \nu(t)\right\rangle=\left\langle v_{-}(t) \mid \nu(t)\right\rangle
\end{array} \quad \text { on }[y=f(t, x)+t V]\right.
$$

where $\nu(t)$ is the unit normal at $[y=f(t, x)+t V]$ pointing into $\Omega_{+}^{V}(t)$ and $\langle\cdot \mid \cdot\rangle$ the inner product in $\mathbb{R}^{2}$. Additionally, we impose the far-field boundary condition

$$
\left.v_{ \pm}(t, x, y) \rightarrow(0, V) \text { for }|y| \rightarrow \infty \text { (uniformly in } x\right) .
$$

The motion of the free interface is described by the kinematic boundary condition

$$
\partial_{t} f(t)=\left\langle v_{ \pm}(t) \mid\left(-f^{\prime}(t), 1\right)\right\rangle-V \quad \text { on }[y=f(t, x)+t V],
$$

\footnotetext{
${ }^{3}$ We write $\dot{f}$ to denote the derivative $\mathrm{d} f / \mathrm{d} t$.
} 
and, since we consider $2 \pi$-periodic flows, $f(t), v_{ \pm}(t)$, and $p_{ \pm}(t)$ are assumed to be $2 \pi$-periodic with respect to $x$ for all $t \geq 0$. Finally, we supplement the system with the initial condition

$$
f(0)=f_{0} .
$$

It is convenient to rewrite the Eq. (2.1) in a reference frame that moves with the constant velocity $(0, V)$. To this end we let

$$
\Omega_{ \pm}(t):=\left\{(x, y) \in \mathbb{R}^{2}: \pm(f(t, x)-y)<0\right\}=\Omega_{ \pm}^{V}(t)-(0, t V),
$$

and

$$
\left\{\begin{array}{l}
P_{ \pm}(t, x, y)=p_{ \pm}(t, x, y+t V) \\
V_{ \pm}(t, x, y)=v_{ \pm}(t, x, y+t V)-(0, V)
\end{array} \quad \text { for } t \geq 0 \text { and }(x, y) \in \Omega_{ \pm}(t)\right.
$$

Direct computations show that (2.1) is equivalent to

$$
\begin{cases}\operatorname{div} V_{ \pm}(t)=0 & \text { in } \Omega_{ \pm}(t), \\ \mu_{ \pm}\left(V_{ \pm}(t)+(0, V)\right)=-k\left(\nabla P_{ \pm}(t)+\left(0, \rho_{ \pm} g\right)\right) & \text { in } \Omega_{ \pm}(t), \\ \left\langle V_{+}(t) \mid \nu(t)\right\rangle=\left\langle V_{-}(t) \mid \nu(t)\right\rangle & \text { on }[y=f(t, x)], \\ P_{+}(t)-P_{-}(t)=\sigma \kappa(f(t)) & \text { on }[y=f(t, x)], \\ V_{ \pm}(t, x, y) \rightarrow 0 & \text { for }|y| \rightarrow \infty, \\ \partial_{t} f(t)=\left\langle V_{ \pm}(t) \mid\left(-f^{\prime}(t), 1\right)\right\rangle & \text { on }[y=f(t, x)], \\ f(0)=f_{0} . & \end{cases}
$$

In Proposition 2.3 we establish the equivalence of the two formulations (1.1) and (2.3). It is important to point out that the function $\bar{\omega}$ in $(1.1 \mathrm{a})_{1}$ is uniquely identified by $f$ in the space $\widehat{L}_{2}(\mathbb{S}$ ) (this feature is established rigorously only later on in Theorem 3.3). This aspect is essential at several places in this paper, see Proposition 2.3 and the preparatory lemma below.

Lemma 2.1. Given $f \in H^{1}(\mathbb{S})$ and $\bar{\omega} \in \widehat{L}_{2}(\mathbb{S})$ let

$$
\begin{aligned}
V^{1}(x, y) & :=-\frac{1}{4 \pi} \int_{\mathbb{S}} \bar{\omega}(s) \frac{\tan h((y-f(s)) / 2)\left[1+\tan ^{2}((x-s) / 2)\right]}{\tan ^{2}((x-s) / 2)+\tan h^{2}((y-f(s)) / 2)} \mathrm{d} s, \\
V^{2}(x, y) & :=\frac{1}{4 \pi} \int_{\mathbb{S}} \bar{\omega}(s) \frac{\tan ((x-s) / 2)\left[1-\tan h^{2}((y-f(s)) / 2)\right]}{\tan ^{2}((x-s) / 2)+\tan h^{2}((y-f(s)) / 2)} \mathrm{d} s
\end{aligned}
$$

for $(x, y) \in \mathbb{R}^{2} \backslash[y=f(x)]$ and set $V:=\left(V^{1}, V^{2}\right)$ and $V_{ \pm}:=\left.V\right|_{\Omega_{ \pm}}$, where

$$
\Omega_{ \pm}:=\left\{(x, y) \in \mathbb{R}^{2}: \pm(f(x)-y)<0\right\} .
$$

Then, there exists a constant $C=C\left(\|f\|_{\infty}\right)>0$ such that

$$
\left|V_{ \pm}(x, y)\right| \leq C\|\bar{\omega}\|_{1} e^{-|y| / 2}
$$

for all $(x, y) \in \Omega_{ \pm}$satisfying $|y| \geq 1+2\|f\|_{\infty}$.

Proof. Let first $f \neq 0$. Taking advantage of

$$
\max \left\{\tan h\left(\frac{\|f\|_{\infty}}{2}\right), \tan h\left(\frac{|y|}{4}\right)\right\} \leq\left|\tan h\left(\frac{y-f(s)}{2}\right)\right| \quad \text { for }|y| \geq 2\|f\|_{\infty},
$$

for $|y| \geq 2\|f\|_{\infty}$, it follows that

$$
\left|V_{ \pm}^{2}(x, y)\right| \leq \frac{\|\bar{\omega}\|_{1}}{\tan h\left(\|f\|_{\infty} / 2\right)}\left|1-\tan h^{2}(y / 4)\right| \leq \frac{\|\bar{\omega}\|_{1}}{\tan h\left(\|f\|_{\infty} / 2\right)} e^{-|y| / 2} .
$$

In order to estimate $V_{ \pm}^{1}$ we use the fact that $\langle\bar{\omega}\rangle=0$ to derive, after performing some elementary estimates, that

$$
\begin{aligned}
\left|V_{ \pm}^{1}(x, y)\right| & \leq \int_{\mathbb{S}}|\bar{\omega}(s)| \frac{\tan h((y-f(s)) / 2)\left[1+\tan ^{2}((x-s) / 2)\right]}{\tan ^{2}((x-s) / 2)+\tan h^{2}((y-f(s)) / 2)} \mp 1 \mid \mathrm{d} s \\
& \leq C\|\bar{\omega}\|_{1}(1-\tan h(|y| / 4)) \leq C\|\bar{\omega}\|_{1} e^{-|y| / 2}
\end{aligned}
$$


for all $|y| \geq 2\|f\|_{\infty}$. The claim for $f=0$ follows in a similar way.

In Proposition 2.3 we show that, given a solution to (1.1), the velocity field in the classical formulation (2.1) at time $t$ can be expressed in terms of $f:=f(t)$ and $\bar{\omega}:=\bar{\omega}(t)$ according to Lemma 2.1, provided that $f$ and $\bar{\omega}$ have suitable regularity properties. We point out that a formal derivation of the formula (2.4) is provided, in a more general context, in [21, Section 2]. In Lemma 2.2 we establish further properties of the velocity field defined in Lemma 2.1 .

Lemma 2.2. Let $f \in H^{2}(\mathbb{S})$ and $\bar{\omega} \in \widehat{H}^{1}(\mathbb{S})$. The vector field $V_{ \pm}$introduced in Lemma 2.1 belongs to $\mathrm{C}\left(\bar{\Omega}_{ \pm}\right) \cap \mathrm{C}^{1}\left(\Omega_{ \pm}\right)$, it is divergence free and irrotational, and

$$
\begin{aligned}
V_{ \pm}(x, f(x))= & \frac{1}{4 \pi} \mathrm{PV} \int_{-\pi}^{\pi} \bar{\omega}(x-s) \frac{\left(-\left(T_{[x, s]} f\right)\left(1+t_{[s]}^{2}\right), t_{[s]}\left[1-\left(T_{[x, s]} f\right)^{2}\right]\right)}{t_{[s]}^{2}+\left(T_{[x, s]} f\right)^{2}} \mathrm{~d} s \\
& \mp \frac{1}{2} \frac{\bar{\omega}(x)\left(1, f^{\prime}(x)\right)}{1+f^{\prime 2}(x)}, \quad x \in \mathbb{R} .
\end{aligned}
$$

Letting further

$$
P_{ \pm}(x, y):=c_{ \pm}-\frac{\mu_{ \pm}}{k} \int_{0}^{x} V_{ \pm}^{1}(s, \pm d) \mathrm{d} s-\frac{\mu_{ \pm}}{k} \int_{ \pm d}^{y} V_{ \pm}^{2}(x, s) \mathrm{d} s-\left(\rho_{ \pm} g+\frac{\mu_{ \pm} V}{k}\right) y
$$

for $(x, y) \in \bar{\Omega}_{ \pm}$, where $c_{ \pm} \in \mathbb{R}$ and $d>\|f\|_{\infty}$, it holds that $P_{ \pm} \in \mathrm{C}^{1}\left(\bar{\Omega}_{ \pm}\right) \cap \mathrm{C}^{2}\left(\Omega_{ \pm}\right)$and the relations $(2.3)_{1}-(2.3)_{3},(2.3)_{5}$ are all satisfied.

Proof. The theorem on the differentiation of parameter integrals shows that $V_{ \pm}$is continuously differentiable in $\Omega_{ \pm}$, divergence free, and irrotational. In order to show that $V_{ \pm} \in \mathrm{C}\left(\bar{\Omega}_{ \pm}\right)$it suffices to show that the one-sided limits when approaching a point $\left(x_{0}, f\left(x_{0}\right)\right) \in[y=f(x)]$ from $\Omega_{-}$and $\Omega_{+}$, respectively, exist. To this end we note that the complex conjugate of $\left(V_{ \pm}^{1}, V_{ \pm}^{2}\right)$ satisfies

$$
\overline{\left(V_{ \pm}^{1}, V_{ \pm}^{2}\right)}(z)=\frac{1}{4 \pi i} \int_{\Gamma} \frac{g(\xi)}{\tan ((\xi-z) / 2)} \mathrm{d} \xi \quad \text { for } z=(x, y) \notin[y=f(x)]
$$

with $\Gamma$ being a $2 \pi$-period of the graph $[y=f(x)]$ and with $g: \Gamma \rightarrow \mathbb{C}$ defined by

$$
g(\xi)=-\frac{\bar{\omega}(s)\left(1-i f^{\prime}(s)\right)}{1+f^{\prime 2}(s)} \quad \text { for } \xi=(s, f(s)) \in \Gamma .
$$

Given $z=(x, y) \notin[y=f(x)]$, it is convenient to write

$$
\overline{\left(V_{ \pm}^{1}, V_{ \pm}^{2}\right)}(z)=\frac{1}{4 \pi i} \int_{\Gamma} g(\xi)\left[\frac{1}{\tan ((\xi-z) / 2)}-\frac{1}{(\xi-z) / 2}\right] \mathrm{d} \xi+\frac{1}{2 \pi i} \int_{\Gamma} \frac{g(\xi)}{\xi-z} \mathrm{~d} \xi,
$$

because Lebesgue's theorem now shows that if $z_{n}$ approaches $z_{0}=\left(x_{0}, f\left(x_{0}\right)\right)$ from $\Omega_{+}$(or $\left.\Omega_{-}\right)$, then

$$
\frac{1}{4 \pi i} \int_{\Gamma} g(\xi)\left[\frac{1}{\tan \left(\left(\xi-z_{n}\right) / 2\right)}-\frac{1}{\left(\xi-z_{n}\right) / 2}\right] \mathrm{d} \xi \underset{n \rightarrow \infty}{\longrightarrow} \frac{1}{4 \pi i} \int_{\Gamma} g(\xi)\left[\frac{1}{\tan \left(\left(\xi-z_{0}\right) / 2\right)}-\frac{1}{\left(\xi-z_{0}\right) / 2}\right] \mathrm{d} \xi
$$

Moreover, using Plemelj's formula, cf. e.g. [45, Theorem 2.5.1], we find that

$$
\frac{1}{2 \pi i} \int_{\Gamma} \frac{g(\xi)}{\xi-z_{n}} \mathrm{~d} \xi \underset{n \rightarrow \infty}{\longrightarrow} \pm \frac{g\left(z_{0}\right)}{2}+\frac{1}{2 \pi i} \mathrm{PV} \int_{\Gamma} \frac{g(\xi)}{\xi-z_{0}} \mathrm{~d} \xi
$$

where the PV is taken at $\xi=z_{0}$, and we conclude that

$$
\overline{\left(V_{ \pm}^{1}, V_{ \pm}^{2}\right)}\left(z_{n}\right) \underset{n \rightarrow \infty}{\longrightarrow} \pm \frac{g\left(z_{0}\right)}{2}+\frac{1}{4 \pi i} \mathrm{PV} \int_{\Gamma} \frac{g(\xi)}{\tan \left(\left(\xi-z_{0}\right) / 2\right)} \mathrm{d} \xi
$$

The formula (2.5) and the property $V_{ \pm} \in \mathrm{C}\left(\bar{\Omega}_{ \pm}\right)$follow at once. The remaining claims are simple consequences of Lemma 2.1 and of the already established properties.

Using Lemmas 2.1 and 2.2, we conclude this section with the following equivalence result. 
Proposition 2.3 (Equivalence of formulations). Let $T \in(0, \infty]$ be given .

(a) Let $\sigma=0$. The following are equivalent:

(i) the problem $(2.3)$ for $f \in \mathrm{C}^{1}\left([0, T), L_{2}(\mathbb{S})\right)$ and

- $f(t) \in H^{2}(\mathbb{S}), \bar{\omega}(t):=\left\langle\left.\left(V_{-}(t)-V_{+}(t)\right)\right|_{[y=f(t, x)]} \mid\left(1, f^{\prime}(t)\right)\right\rangle \in \widehat{H}^{1}(\mathbb{S})$,

- $V_{ \pm}(t) \in \mathrm{C}\left(\bar{\Omega}_{ \pm}(t)\right) \cap \mathrm{C}^{1}\left(\Omega_{ \pm}(t)\right), P_{ \pm}(t) \in \mathrm{C}^{1}\left(\bar{\Omega}_{ \pm}(t)\right) \cap \mathrm{C}^{2}\left(\Omega_{ \pm}(t)\right)$

for all $t \in[0, T)$;

(ii) the evolution problem (1.1) for $f \in \mathrm{C}^{1}\left([0, T), L_{2}(\mathbb{S})\right), f(t) \in H^{2}(\mathbb{S})$, and $\bar{\omega}(t) \in \widehat{H}^{1}(\mathbb{S})$ for all $t \in[0, T)$.

(b) Let $\sigma>0$. The following are equivalent:

(i) the problem $(2.3)$ for $f \in \mathrm{C}^{1}\left((0, T), L_{2}(\mathbb{S})\right) \cap \mathrm{C}\left([0, T), L_{2}(\mathbb{S})\right)$ and

- $f(t) \in H^{4}(\mathbb{S}), \bar{\omega}(t):=\left\langle\left.\left(V_{-}(t)-V_{+}(t)\right)\right|_{[y=f(t, x)]} \mid\left(1, f^{\prime}(t)\right)\right\rangle \in \widehat{H}^{1}(\mathbb{S})$,

- $V_{ \pm}(t) \in \mathrm{C}\left(\bar{\Omega}_{ \pm}(t)\right) \cap \mathrm{C}^{1}\left(\Omega_{ \pm}(t)\right), P_{ \pm}(t) \in \mathrm{C}^{1}\left(\bar{\Omega}_{ \pm}(t)\right) \cap \mathrm{C}^{2}\left(\Omega_{ \pm}(t)\right)$

for all $t \in(0, T)$;

(ii) the Muskat problem (1.1) for $f \in \mathrm{C}^{1}\left((0, T), L_{2}(\mathbb{S})\right) \cap \mathrm{C}\left([0, T), L_{2}(\mathbb{S})\right), f(t) \in H^{4}(\mathbb{S})$, and $\bar{\omega}(t) \in$ $\widehat{H}^{1}(\mathbb{S})$ for all $t \in(0, T)$.

Proof. To prove the implication $(i) \Rightarrow(i i)$ of $(a)$, let $\left(f, V_{ \pm}, P_{ \pm}\right)$be a solution to $(2.3)$ on $[0, T)$ and choose $t \geq 0$ fixed but arbitrary (the time dependence is not written explicitly in this proof). Letting

$$
\omega:=\partial_{x} V^{2}-\partial_{y} V^{1} \in \mathcal{D}^{\prime}\left(\mathbb{R}^{2}\right)
$$

denote the vorticity associated to the global velocity field

$$
\left(V^{1}, V^{2}\right):=V_{-} \mathbf{1}_{\Omega_{-}}+V_{+} \mathbf{1}_{\Omega_{+}},
$$

where $\mathbf{1}_{\Omega_{ \pm}}$is the characteristic function of $\Omega_{ \pm}$, it follows from $(2.3)_{3}$ and Stokes' theorem that

$$
\omega=\bar{\omega} \delta_{[y=f(x)]},
$$

where

$$
\bar{\omega}:=\left\langle\left.\left(V_{-}-V_{+}\right)\right|_{[y=f(x)]} \mid\left(1, f^{\prime}\right)\right\rangle \in \widehat{H}^{1}(\mathbb{S}) .
$$

Similarly as in the particular case $\mu_{-}=\mu_{+}$, cf. [47, Proposition 2.2], we find that the global velocity field $\left(V^{1}, V^{2}\right)$ is given by (2.4). Lemma 2.2 now shows, together with the kinematic boundary condition, that $f$ solves the Eq. $(1.1 \mathrm{a})_{1}$. Besides, differentiating the Laplace-Young Eq. $(2.3)_{4}$, the relations $(2.3)_{2}$ and (2.5) finally lead us to (1.1a) $)_{2}$, and the proof of this implication is complete.

For the reverse implication, we define $V_{ \pm}$according to (2.4), and the pressures by (2.6). For suitable $c_{ \pm}$, it follows from $(1.1 \mathrm{a})_{2}$ and Lemmas 2.1, 2.2 that indeed $\left(f, V_{ \pm}, P_{ \pm}\right)$solves $(2.3)$.

The equivalence stated at $(b)$ follows in a similar way.

\section{The Double Layer Potential and Its Adjoint}

We point out that the Eq. (1.1a $)_{2}$ is linear with respect to $\bar{\omega}(t)$. The main goal of this section is to address the solvability of this equation for $\bar{\omega}(t)$ in suitable function spaces, cf. Theorems 3.3 and 3.5 . To this end we first associate to (1.1a) two singular operators and study their mapping properties (see Lemmas 3.1 and 3.2). Finally, in Theorem 3.6 and Lemma 3.7 we study the properties of the adjoints of these singular operators.

To begin, we write $(1.1 \mathrm{a})_{2}$ in the more compact form

$$
\left(1+a_{\mu} \mathbb{A}(f)\right)[\bar{\omega}]=\frac{2 k}{\mu_{-}+\mu_{+}}(\sigma \kappa(f)-\Theta f)^{\prime},
$$


where $\mathbb{A}(f)$ is the linear operator

$$
\mathbb{A}(f)[\bar{\omega}](x):=\frac{1}{2 \pi} \mathrm{PV} \int_{-\pi}^{\pi} \frac{f^{\prime}(x) t_{[s]}\left[1-\left(T_{[x, s]} f\right)^{2}\right]-\left(1+t_{[s]}^{2}\right) T_{[x, s]} f}{t_{[s]}^{2}+\left(T_{[x, s]} f\right)^{2}} \bar{\omega}(x-s) \mathrm{d} s .
$$

Given $f \in H^{r}(\mathbb{S})$ with $r>3 / 2$, we prove in Lemma 3.2 that $\mathbb{A}(f) \in \mathcal{L}\left(L_{2}(\mathbb{S})\right)$. Then, it is a matter of direct computation to verify that $\mathbb{A}(f)$ is the $L_{2}$-adjoint of the double layer potential

$$
(\mathbb{A}(f))^{*}[\xi](x):=\frac{1}{2 \pi} \mathrm{PV} \int_{-\pi}^{\pi} \frac{\left(1+t_{[s]}^{2}\right)\left(T_{[x, s]} f\right)-f^{\prime}(x-s) t_{[s]}\left[1-\left(T_{[x, s]} f\right)^{2}\right]}{t_{[s]}^{2}+\left(T_{[x, s]} f\right)^{2}} \xi(x-s) \mathrm{d} s .
$$

A main part of the subsequent analysis is devoted to the study of the invertibility of the linear operator $1+a_{\mu} \mathbb{A}(f)$ in the algebras $\mathcal{L}\left(\widehat{L}_{2}(\mathbb{S})\right)$ and $\mathcal{L}\left(\widehat{H}^{1}(\mathbb{S})\right)$. These invertibility properties enable us to solve $(3.1)$ and to formulate (1.1) as an evolution equation for $f$ only, that is

$$
\partial_{t} f=\frac{k}{\mu_{-}+\mu_{+}} \mathbb{B}(f)\left[\left(1+a_{\mu} \mathbb{A}(f)\right)^{-1}\left[(\sigma \kappa(f)-\Theta f)^{\prime}\right]\right],
$$

where we have associated to $(1.1 \mathrm{a})_{1}$ the operator $\mathbb{B}(f)$ defined by

$$
\mathbb{B}(f)[\bar{\omega}](x):=\frac{1}{2 \pi} \mathrm{PV} \int_{-\pi}^{\pi} \frac{f^{\prime}(x)\left(1+t_{[s]}^{2}\right)\left(T_{[x, s]} f\right)+t_{[s]}\left[1-\left(T_{[x, s]} f\right)^{2}\right]}{t_{[s]}^{2}+\left(T_{[x, s]} f\right)^{2}} \bar{\omega}(x-s) \mathrm{d} s .
$$

As a first result we establish the following mapping properties.

Lemma 3.1. Given $r>3 / 2$, it holds that

$$
\mathbb{B} \in \mathrm{C}^{\omega}\left(H^{r}(\mathbb{S}), \mathcal{L}\left(\widehat{L}_{2}(\mathbb{S})\right)\right) \cap \mathrm{C}^{\omega}\left(H^{2}(\mathbb{S}), \mathcal{L}\left(\widehat{H}^{1}(\mathbb{S})\right)\right) .
$$

Proof. Let us first assume that

$$
\mathbb{B} \in \mathrm{C}^{\omega}\left(H^{r}(\mathbb{S}), \mathcal{L}\left(L_{2}(\mathbb{S})\right)\right) \cap \mathrm{C}^{\omega}\left(H^{2}(\mathbb{S}), \mathcal{L}\left(H^{1}(\mathbb{S})\right)\right) .
$$

Given $f, \bar{\omega} \in \mathrm{C}^{\infty}(\mathbb{S})$ with $\langle\bar{\omega}\rangle=0$ let $V_{-}$be as defined in Lemma 2.1. Observing that

$$
\mathbb{B}(f)[\bar{\omega}]=2\left\langle\left. V_{-}\right|_{[y=f(x)]} \mid\left(-f^{\prime}, 1\right)\right\rangle \in \mathrm{C}(\mathbb{S}),
$$

Stokes' formula together with Lemmas 2.1, 2.2 yields

$$
\frac{1}{2}\langle\mathbb{B}(f)[\bar{\omega}]\rangle=\int_{\Gamma}\left\langle V_{-} \mid \nu\right\rangle \mathrm{d} \sigma=\int_{\Omega_{-}} \operatorname{div} V_{-} \mathrm{d}(x, y)=0,
$$

and therefore $\mathbb{B}(f)[\bar{\omega}] \in \widehat{L}_{2}(\mathbb{S})$. This immediately implies (3.6).

Hence, we are left to establish (3.7). To this end it is convenient to write

$$
\mathbb{B}(f)=f^{\prime} \mathbb{B}_{1}(f)-\mathbb{B}_{2}(f)+\mathbb{B}_{3}(f),
$$

where

$$
\begin{aligned}
\mathbb{B}_{1}(f)[\bar{\omega}](x):= & \frac{1}{2 \pi} \int_{-\pi}^{\pi} \frac{t_{[s]}^{2} T_{[x, s]} f}{t_{[s]}^{2}+\left(T_{[x, s]} f\right)^{2}} \bar{\omega}(x-s) \mathrm{d} s \\
& +\frac{1}{2 \pi} \int_{-\pi}^{\pi}\left[\frac{T_{[x, s]} f}{t_{[s]}^{2}+\left(T_{[x, s]} f\right)^{2}}-\frac{\left(\delta_{[x, s]} f / 2\right)}{(s / 2)^{2}+\left(\delta_{[x, s]} f / 2\right)^{2}}\right] \bar{\omega}(x-s) \mathrm{d} s, \\
\mathbb{B}_{2}(f)[\bar{\omega}](x):= & \frac{1}{2 \pi} \int_{-\pi}^{\pi} \frac{t_{[s]}\left(T_{[x, s]} f\right)^{2}}{t_{[s]}^{2}+\left(T_{[x, s]} f\right)^{2}} \bar{\omega}(x-s) \mathrm{d} s \\
& -\frac{1}{2 \pi} \int_{-\pi}^{\pi}\left[\frac{t_{[s]}}{t_{[s]}^{2}+\left(T_{[x, s]} f\right)^{2}}-\frac{s / 2}{(s / 2)^{2}+\left(\delta_{[x, s]} f / 2\right)^{2}}\right] \bar{\omega}(x-s) \mathrm{d} s, \\
\mathbb{B}_{3}(f)[\bar{\omega}](x):= & \frac{1}{\pi} \mathrm{PV} \int_{-\pi}^{\pi} \frac{s+f^{\prime}(x)\left(\delta_{[x, s]} f\right)}{s^{2}+\left(\delta_{[x, s]} f\right)^{2}} \bar{\omega}(x-s) \mathrm{d} s .
\end{aligned}
$$


Taking advantage of the relations

$$
\begin{aligned}
& \tan h(x) \leq x, x \geq 0, \quad x \leq \tan (x), x \in[0, \pi / 2), \\
& |\tan h(x)-x| \leq|x|^{3}, x \in \mathbb{R}, \quad|\tan (x)-x| \leq|x| \tan ^{2}(x),|x|<\pi / 2,
\end{aligned}
$$

it is easy to see that $\mathbb{B}_{i}(f) \in \mathcal{L}\left(L_{2}(\mathbb{S}), L_{\infty}(\mathbb{S})\right)$ for $i \in\{1,2\}$ (and that PV is not needed). In fact these mappings are real-analytic, that is

$$
\mathbb{B}_{i} \in \mathrm{C}^{\omega}\left(H^{r}(\mathbb{S}), \mathcal{L}\left(L_{2}(\mathbb{S}), L_{\infty}(\mathbb{S})\right)\right), \quad i \in\{1,2\} .
$$

Furthermore, given $\tau \in(1 / 2,1)$, classical (but lengthy) arguments (see [47, Lemmas 3.2-3.3] where similar integral operators are discussed) show that

$$
\mathbb{B}_{i} \in \mathrm{C}^{\omega}\left(H^{r}(\mathbb{S}), \mathcal{L}\left(H^{\tau}(\mathbb{S}), \mathrm{C}^{1}(\mathbb{S})\right)\right), \quad i \in\{1,2\},
$$

and we are left to consider the operator $\mathbb{B}_{3}$.

Recalling Lemma A.1, we see that

$$
\pi \mathbb{B}_{3}(f)[\bar{\omega}]=C_{0,1}(f)[\bar{\omega}]+f^{\prime} C_{1,1}(f)[f, \bar{\omega}],
$$

and Lemma A.1 $(i)$ immediately yields $\mathbb{B}_{3}(f) \in \mathcal{L}\left(L_{2}(\mathbb{S})\right)$. Moreover, arguing as in [49, Section 5], it follows that

$$
\mathbb{B}_{3} \in \mathrm{C}^{\omega}\left(H^{r}(\mathbb{S}), \mathcal{L}\left(L_{2}(\mathbb{S})\right)\right) .
$$

In order to prove that $\mathbb{B}_{3}(f) \in \mathcal{L}\left(H^{1}(\mathbb{S})\right)$, when additionally $f \in H^{2}(\mathbb{S})$, we let $\left\{\tau_{\varepsilon}\right\}_{\varepsilon \in \mathbb{R}}$ denote the $C_{0^{-}}$ group of right translations, that is $\tau_{\varepsilon} h(x)=h(x-\varepsilon)$ for $x \in \mathbb{R}$ and $h \in L_{2}(\mathbb{S})$. Given $\varepsilon>0$ and $\bar{\omega} \in H^{1}(\mathbb{S})$, it holds that

$$
\begin{aligned}
\pi \frac{\tau_{\varepsilon}\left(\mathbb{B}_{3}(f)[\bar{\omega}]\right)-\mathbb{B}_{3}(f)[\bar{\omega}]}{\varepsilon}= & C_{0,1}\left(\tau_{\varepsilon} f\right)\left[\frac{\tau_{\varepsilon} \bar{\omega}-\bar{\omega}}{\varepsilon}\right]-C_{2,2}\left(f, \tau_{\varepsilon} f\right)\left[\frac{\tau_{\varepsilon} f-f}{\varepsilon}, \tau_{\varepsilon} f+f, \bar{\omega}\right] \\
& +\frac{\tau_{\varepsilon} f^{\prime}-f^{\prime}}{\varepsilon} C_{1,1}\left(\tau_{\varepsilon} f\right)\left[\tau_{\varepsilon} f, \tau_{\varepsilon} \bar{\omega}\right]+f^{\prime} C_{1,1}\left(\tau_{\varepsilon} f\right)\left[\tau_{\varepsilon} f, \frac{\tau_{\varepsilon} \bar{\omega}-\bar{\omega}}{\varepsilon}\right] \\
& +f^{\prime} C_{1,1}\left(\tau_{\varepsilon} f\right)\left[\frac{\tau_{\varepsilon} f-f}{\varepsilon}, \bar{\omega}\right]-f^{\prime} C_{3,2}\left(f, \tau_{\varepsilon} f\right)\left[\frac{\tau_{\varepsilon} f-f}{\varepsilon}, \tau_{\varepsilon} f+f, f, \bar{\omega}\right] .
\end{aligned}
$$

Since

$$
\begin{array}{ll}
\tau_{\varepsilon} \bar{\omega} \underset{\varepsilon \rightarrow 0}{\longrightarrow} \bar{\omega} \quad \text { in } H^{1}(\mathbb{S}), & \tau_{\varepsilon} f \underset{\varepsilon \rightarrow 0}{\longrightarrow} f \quad \text { in } H^{2}(\mathbb{S}), \\
\frac{\tau_{\varepsilon} \bar{\omega}-\bar{\omega}}{\varepsilon} \underset{\varepsilon \rightarrow 0}{\longrightarrow}-\bar{\omega}^{\prime} \quad \text { in } L_{2}(\mathbb{S}), & \frac{\tau_{\varepsilon} f-f}{\varepsilon} \underset{\varepsilon \rightarrow 0}{\longrightarrow}-f^{\prime} \quad \text { in } H^{1}(\mathbb{S}),
\end{array}
$$

we may pass, in view of Lemma A.1 (i)-(iii), to the limit $\varepsilon \rightarrow 0$ in the identity above to conclude that

$$
\begin{aligned}
-\pi \frac{\tau_{\varepsilon}\left(\mathbb{B}_{3}(f)[\bar{\omega}]\right)-\mathbb{B}_{3}(f)[\bar{\omega}]}{\varepsilon} \underset{\varepsilon \rightarrow 0}{\longrightarrow} C_{0,1}(f)\left[\bar{\omega}^{\prime}\right]-2 C_{2,2}(f, f)\left[f^{\prime}, f, \bar{\omega}\right]+f^{\prime \prime} C_{1,1}(f)[f, \bar{\omega}]+f^{\prime} C_{1,1}(f)\left[f, \bar{\omega}^{\prime}\right] \\
+f^{\prime} C_{1,1}(f)\left[f^{\prime}, \bar{\omega}\right]-2 f^{\prime} C_{3,2}(f, f)\left[f^{\prime}, f, f, \bar{\omega}\right]
\end{aligned}
$$

in $L_{2}(\mathbb{S})$. This proves that $\mathbb{B}_{3}(f)[\bar{\omega}] \in H^{1}(\mathbb{S})$, with

$$
\begin{aligned}
\pi\left(\mathbb{B}_{3}(f)[\bar{\omega}]\right)^{\prime}= & \pi \mathbb{B}_{3}(f)\left[\bar{\omega}^{\prime}\right]-2 C_{2,2}(f, f)\left[f^{\prime}, f, \bar{\omega}\right]+f^{\prime \prime} C_{1,1}(f)[f, \bar{\omega}] \\
& +f^{\prime} C_{1,1}(f)\left[f^{\prime}, \bar{\omega}\right]-2 f^{\prime} C_{3,2}(f, f)\left[f^{\prime}, f, f, \bar{\omega}\right] .
\end{aligned}
$$

Lemma A.1 and the arguments in [49, Section 5] finally lead us to

$$
\mathbb{B}_{3} \in \mathrm{C}^{\omega}\left(H^{2}(\mathbb{S}), \mathcal{L}\left(H^{1}(\mathbb{S})\right)\right),
$$

and (3.7) follows now from (3.9), (3.11) and (3.13). This completes the proof.

We now study the mapping properties of the operator $\mathbb{A}$ introduced in (3.2).

Lemma 3.2. Let $r>3 / 2$ be given. It then holds

$$
\mathbb{A} \in \mathrm{C}^{\omega}\left(H^{r}(\mathbb{S}), \mathcal{L}\left(\widehat{L}_{2}(\mathbb{S})\right)\right) \cap \mathrm{C}^{\omega}\left(H^{2}(\mathbb{S}), \mathcal{L}\left(\widehat{H}^{1}(\mathbb{S})\right)\right) .
$$


Proof. Pick first $f, \bar{\omega} \in \mathrm{C}^{\infty}(\mathbb{S})$ with $\langle\bar{\omega}\rangle=0$ and let $V_{-} \in \mathrm{C}\left(\bar{\Omega}_{-}\right) \cap \mathrm{C}^{1}\left(\Omega_{-}\right)$be as defined in Lemma 2.1. It then holds

$$
2\left\langle\left. V_{-}\right|_{[y=f(x)]} \mid\left(1, f^{\prime}\right)\right\rangle=(1+\mathbb{A}(f))[\bar{\omega}] \in \mathrm{C}(\mathbb{S}),
$$

and therefore $\mathbb{A}(f)[\bar{\omega}] \in \widehat{L}_{2}(\mathbb{S})$ if and only if $\left\langle\left. V_{-}\right|_{[y=f(x)]} \mid\left(1, f^{\prime}\right)\right\rangle \in \widehat{L}_{2}(\mathbb{S})$. The latter property follows from the periodicity of $f$ and $P_{-}$, where $P_{-} \in \mathrm{C}^{1}\left(\bar{\Omega}_{-}\right)$is given in (2.6), with respect to $x$ and the relation

$$
V_{-}=-\frac{k}{\mu_{-}}\left(\nabla P_{-}+\left(0, \rho_{-} g\right)\right)-(0, V) \text { in } \bar{\Omega}_{-} .
$$

We are thus left to prove that

$$
\mathbb{A} \in \mathrm{C}^{\omega}\left(H^{r}(\mathbb{S}), \mathcal{L}\left(L_{2}(\mathbb{S})\right)\right) \cap \mathrm{C}^{\omega}\left(H^{2}(\mathbb{S}), \mathcal{L}\left(H^{1}(\mathbb{S})\right)\right) .
$$

We proceed as in the previous lemma and write

$$
\mathbb{A}(f)=-f^{\prime} \mathbb{B}_{2}(f)-\mathbb{B}_{1}(f)+\mathbb{A}_{3}(f),
$$

where, using the notation introduced in Lemma A.1, we have

$$
\pi \mathbb{A}_{3}(f)[\bar{\omega}]=f^{\prime} C_{0,1}(f)[\bar{\omega}]-C_{1,1}(f)[f, \bar{\omega}] .
$$

Similarly as in Lemma 3.1, we get

$$
\mathbb{A}_{3} \in \mathrm{C}^{\omega}\left(H^{r}(\mathbb{S}), \mathcal{L}\left(L_{2}(\mathbb{S})\right)\right) \cap \mathrm{C}^{\omega}\left(H^{2}(\mathbb{S}), \mathcal{L}\left(H^{1}(\mathbb{S})\right)\right),
$$

with

$$
\begin{aligned}
\pi\left(\mathbb{A}_{3}(f)[\bar{\omega}]\right)^{\prime}= & \pi \mathbb{A}_{3}(f)\left[\bar{\omega}^{\prime}\right]+f^{\prime \prime} C_{0,1}(f)[\bar{\omega}]-2 f^{\prime} C_{2,2}(f, f)\left[f^{\prime}, f, \bar{\omega}\right] \\
& -C_{1,1}(f)\left[f^{\prime}, \bar{\omega}\right]+2 f^{\prime} C_{3,2}(f, f)\left[f^{\prime}, f, f, \bar{\omega}\right] .
\end{aligned}
$$

The properties (3.9), (3.10), and (3.17) combined imply (3.15), and the proof is complete.

We now address the solvability of Eq. (3.1). To this end we first establish the invertibility of $1+a_{\mu} \mathbb{A}(f)$ in $\mathcal{L}\left(\widehat{L}_{2}(\mathbb{S})\right)$. A similar property has been established, under stronger assumptions on $f$ which are not compatible with our approach, in [21].

Theorem 3.3. Let $r>3 / 2$ and $M>0$. Then, there exists a constant $C=C(M)>0$ such that

$$
\|\bar{\omega}\|_{2} \leq C\|(\lambda-\mathbb{A}(f))[\bar{\omega}]\|_{2}
$$

for all $\lambda \in \mathbb{R}$ with $|\lambda| \geq 1, \bar{\omega} \in \widehat{L}_{2}(\mathbb{S})$, and $f \in H^{r}(\mathbb{S})$ with $\left\|f^{\prime}\right\|_{\infty} \leq M$.

In particular, $\{\lambda \in \mathbb{R}:|\lambda| \geq 1\}$ is contained in the resolvent set $\mathbb{A}(f) \in \mathcal{L}\left(\widehat{L}_{2}(\mathbb{S})\right)$ for each $f \in H^{r}(\mathbb{S})$.

Proof. In view of Lemma 3.2, it suffices to establish the estimate $(3.19)$ for $\bar{\omega}, f \in \mathrm{C}^{\infty}(\mathbb{S})$ with $\langle\bar{\omega}\rangle=0$ and $\left\|f^{\prime}\right\|_{\infty} \leq M$. Let $V_{ \pm} \in \mathrm{C}\left(\bar{\Omega}_{ \pm}\right) \cap \mathrm{C}^{1}\left(\Omega_{ \pm}\right)$be as defined in Lemma 2.1 and set

$$
F_{ \pm}:=\left(F_{ \pm}^{1}, F_{ \pm}^{2}\right):=\left.V_{ \pm}\right|_{[y=f(x)]} .
$$

We denote by $\tau$ and $\nu$ the tangent and the outward normal unit vectors at $\partial \Omega_{-}$and we decompose $F_{ \pm}$ in tangential and normal components $F_{ \pm}=F_{ \pm}^{\tau}+F_{ \pm}^{\nu}$, where

$$
F_{ \pm}^{\tau}=\mp \frac{(1 \mp \mathbb{A}(f))[\bar{\omega}]}{2\left(1+f^{\prime 2}\right)}\left(1, f^{\prime}\right), \quad F_{ \pm}^{\nu}=\frac{\mathbb{B}(f)[\bar{\omega}]}{2\left(1+f^{\prime 2}\right)}\left(-f^{\prime}, 1\right),
$$

cf. (2.5). Recalling the Lemmas 3.1, 3.2, we may view $F_{ \pm}^{\tau}$ and $F_{ \pm}^{\nu}$ as being elements of $L_{2}\left(\mathbb{S}, \mathbb{R}^{2}\right)$.

We next introduce the bilinear form $\mathcal{B}: L_{2}\left(\mathbb{S}, \mathbb{R}^{2}\right) \times L_{2}\left(\mathbb{S}, \mathbb{R}^{2}\right) \rightarrow \mathbb{R}$ by the formula

$$
\mathcal{B}(F, G):=\int_{\mathbb{S}} G^{2}\left\langle F \mid\left(-f^{\prime}, 1\right)\right\rangle+F^{2}\left\langle G \mid\left(-f^{\prime}, 1\right)\right\rangle-\langle F \mid G\rangle \mathrm{d} x
$$


for $F=\left(F^{1}, F^{2}\right), G=\left(G^{1}, G^{2}\right) \in L_{2}\left(\mathbb{S}, \mathbb{R}^{2}\right)$. Inserting the vector fields $F_{ \pm}$in (3.20), we find by using Lebesgue's dominated convergence theorem, Stokes' formula, and the Lemmas 2.1, 2.2 that

$$
\mathcal{B}\left(F_{ \pm}, F_{ \pm}\right)=\int_{\Gamma}\left\langle\left(\begin{array}{c}
2 F_{ \pm}^{1} F_{ \pm}^{2} \\
\left(F_{ \pm}^{2}\right)^{2}-\left(F_{ \pm}^{1}\right)^{2}
\end{array}\right) \mid \nu\right\rangle \mathrm{d} \sigma=\mp \int_{\Omega_{ \pm}} \operatorname{div}\left(\begin{array}{c}
2 V_{ \pm}^{1} V_{ \pm}^{2} \\
\left(V_{ \pm}^{2}\right)^{2}-\left(V_{ \pm}^{1}\right)^{2}
\end{array}\right) \mathrm{d}(x, y)=0,
$$

where $\Gamma$ denotes again a period of the graph $[y=f(x)]$. Moreover, in virtue of (3.21), we may write (3.22) equivalently as

$$
\int_{\mathbb{S}} \frac{1}{1+f^{\prime 2}}\left[|\mathbb{B}(f)[\bar{\omega}]|^{2} \mp 2 f^{\prime}(\mathbb{B}(f)[\bar{\omega}])(1 \mp \mathbb{A}(f))[\bar{\omega}]-|(1 \mp \mathbb{A}(f))[\bar{\omega}]|^{2}\right] \mathrm{d} x=0,
$$

and, recalling that $\left\|f^{\prime}\right\|_{\infty} \leq M$, we infer from (3.23) that

$$
\|(1 \pm \mathbb{A}(f))[\bar{\omega}]\|_{2} \leq C\|\mathbb{B}(f)[\bar{\omega}]\|_{2},
$$

with a positive constant $C=C(M)$. In particular we get

$$
\|\bar{\omega}\|_{2}=\frac{1}{2}\|(1+\mathbb{A}(f))[\bar{\omega}]+(1-\mathbb{A}(f))[\bar{\omega}]\|_{2} \leq C\|\mathbb{B}(f)[\bar{\omega}]\|_{2} .
$$

Given $\lambda \in \mathbb{R}$ with $|\lambda| \geq 1$, it holds that

$$
|(1 \mp \mathbb{A}(f))[\bar{\omega}]|^{2}=|(\lambda-\mathbb{A}(f))[\bar{\omega}]|^{2}-2(\lambda \mp 1) \bar{\omega}(\lambda-\mathbb{A}(f))[\bar{\omega}]+(\lambda \mp 1)^{2}|\bar{\omega}|^{2},
$$

and eliminating the mixed term on the right hand side we obtain together with (3.23) that

$$
\int_{\mathbb{S}} \frac{1}{1+f^{\prime 2}}\left[\left(\lambda^{2}-1\right)|\bar{\omega}|^{2}+|\mathbb{B}(f)[\bar{\omega}]|^{2}-|(\lambda-\mathbb{A}(f))[\bar{\omega}]|^{2}-2 f^{\prime}(\mathbb{B}(f)[\bar{\omega}])(\lambda-\mathbb{A}(f))[\bar{\omega}]\right] \mathrm{d} x=0,
$$

from where we conclude that

$$
\left(\lambda^{2}-1\right)\|\bar{\omega}\|_{2}+\|\mathbb{B}(f)[\bar{\omega}]\|_{2} \leq C\|(\lambda-\mathbb{A}(f)) \bar{\omega}\|_{2},
$$

with a constant $C=C(M)$. The latter estimate and (3.24) yield (3.19). That $\{\lambda \in \mathbb{R}:|\lambda| \geq 1\}$ belongs to the resolvent set of $\mathbb{A}(f) \in \mathcal{L}\left(\widehat{L}_{2}(\mathbb{S})\right)$ for all $f \in H^{r}(\mathbb{S})$ is a straightforward consequence of (3.19), Lemma 3.2, and of the continuity method, cf. e.g. [5, Proposition I.1.1.1].

The following remark is relevant in Sect. 6 in the stability analysis of the Muskat problem.

Remark 3.4. The estimate

$$
\|\bar{\omega}\|_{2} \leq C\|\mathbb{B}(f)[\bar{\omega}]\|_{2}
$$

derived in (3.24) enables us to identify the equilibrium solutions to the Muskat problem (1.1) (see (3.4)) as being the solutions to the capillarity equation

$$
(\sigma \kappa(f)-\Theta f)^{\prime}=0
$$

We now establish the invertibility of $1+a_{\mu} \mathbb{A}(f)$ in the algebra $\mathcal{L}\left(\widehat{H}^{1}(\mathbb{S})\right)$ under the assumption that $f \in H^{2}(\mathbb{S})$.

Theorem 3.5. Let $M>0$. Then, there exists a constant $C=C(M)>0$ such that

$$
\|\bar{\omega}\|_{H^{1}} \leq C\|(\lambda-\mathbb{A}(f))[\bar{\omega}]\|_{H^{1}}
$$

for all $\lambda \in \mathbb{R}$ with $|\lambda| \geq 1, \bar{\omega} \in \widehat{H}^{1}(\mathbb{S})$, and $f \in H^{2}(\mathbb{S})$ with $\|f\|_{H^{2}} \leq M$.

In particular, $\{\lambda \in \mathbb{R}:|\lambda| \geq 1\}$ is contained in the resolvent set of $\mathbb{A}(f) \in \mathcal{L}\left(\widehat{H}^{1}(\mathbb{S})\right)$ for each $f \in H^{2}(\mathbb{S})$. 
Proof. Recalling (3.19), we are left to estimate the term $\left\|((\lambda-\mathbb{A}(f))[\bar{\omega}])^{\prime}\right\|_{2}$ suitably. To this end, we infer from (3.16) and (3.18) that

$$
(\mathbb{A}(f)[\bar{\omega}])^{\prime}=\mathbb{A}(f)\left[\bar{\omega}^{\prime}\right]+T_{\mathrm{lot}}^{A}(f)[\bar{\omega}],
$$

where the operator $T_{\text {lot }}^{A}(f)$ defined by

$$
\begin{aligned}
\pi T_{\mathrm{lot}}^{A}(f)[\bar{\omega}]:= & f^{\prime \prime} C_{0,1}(f)[\bar{\omega}]-2 f^{\prime} C_{2,2}(f, f)\left[f^{\prime}, f, \bar{\omega}\right]-C_{1,1}(f)\left[f^{\prime}, \bar{\omega}\right] \\
& +2 f^{\prime} C_{3,2}(f, f)\left[f^{\prime}, f, f, \bar{\omega}\right]-\pi\left(\left(f^{\prime} \mathbb{B}_{2}(f)[\bar{\omega}]\right)^{\prime}-f^{\prime} \mathbb{B}_{2}(f)\left[\bar{\omega}^{\prime}\right]\right) \\
& -\pi\left(\left(\mathbb{B}_{1}(f)[\bar{\omega}]\right)^{\prime}-\mathbb{B}_{1}(f)\left[\bar{\omega}^{\prime}\right]\right),
\end{aligned}
$$

encompasses all lower order terms of $\left(\mathbb{A}_{3}(f)[\bar{\omega}]\right)^{\prime}$ with respect to $\bar{\omega}$ as, for each $\tau \in(1 / 2,1)$ fixed, it holds

$$
\left\|T_{\text {lot }}^{A}(f)[\bar{\omega}]\right\|_{2} \leq C\|\bar{\omega}\|_{H^{\tau}} \quad \text { for all } \bar{\omega} \in \widehat{H}^{1}(\mathbb{S}),
$$

with $C=C(M)$. Indeed, letting $r:=(9-2 \tau) / 4$, it follows that $r \in(3 / 2,2)$ and $\tau \in(5 / 2-r, 1)$, and the estimates (A.1) and (A.3) yield

$$
\left\|f^{\prime \prime} C_{0,1}(f)[\bar{\omega}]-2 f^{\prime} C_{2,2}(f, f)\left[f^{\prime}, f, \bar{\omega}\right]-C_{1,1}(f)\left[f^{\prime}, \bar{\omega}\right]+2 f^{\prime} C_{3,2}(f, f)\left[f^{\prime}, f, f, \bar{\omega}\right]\right\|_{2} \leq C\|\bar{\omega}\|_{H^{\tau}} .
$$

Moreover, it follows from (3.9), (3.10) and the compactness of the embedding $H^{2}(\mathbb{S}) \hookrightarrow H^{r}(\mathbb{S})$ that also

$$
\left\|\left(f^{\prime} \mathbb{B}_{2}(f)[\bar{\omega}]\right)^{\prime}\right\|_{2}+\left\|\left(\mathbb{B}_{1}(f)[\bar{\omega}]\right)^{\prime}\right\|_{2} \leq C\|\bar{\omega}\|_{H^{\tau}} .
$$

Finally, using integration by parts in the formulas defining $\mathbb{B}_{1}(f)$ and $\mathbb{B}_{2}(f)$ we get

$$
\left\|f^{\prime} \mathbb{B}_{2}(f)\left[\bar{\omega}^{\prime}\right]\right\|_{2}+\left\|\mathbb{B}_{1}(f)\left[\bar{\omega}^{\prime}\right]\right\|_{2} \leq C\|\bar{\omega}\|_{2},
$$

and (3.29) follows.

Invoking (3.19) and (3.29) we find a constant $c=c(M) \in(0,1)$ with

$$
\begin{aligned}
2\|(\lambda-\mathbb{A}(f))[\bar{\omega}]\|_{H^{1}} & \geq\|(\lambda-\mathbb{A}(f))[\bar{\omega}]\|_{2}+\left\|((\lambda-\mathbb{A}(f))[\bar{\omega}])^{\prime}\right\|_{2} \\
& \geq\|(\lambda-\mathbb{A}(f))[\bar{\omega}]\|_{2}+\left\|(\lambda-\mathbb{A}(f))\left[\bar{\omega}^{\prime}\right]\right\|_{2}-\left\|T_{\mathrm{lot}}^{A}(f)[\bar{\omega}]\right\|_{2} \\
& \geq c\|\bar{\omega}\|_{H^{1}}-\frac{1}{c}\|\bar{\omega}\|_{H^{\tau}},
\end{aligned}
$$

and since by $(3.30)^{4}$ and Young's inequality

$$
\|\bar{\omega}\|_{H^{\tau}} \leq\|\bar{\omega}\|_{2}^{1-\tau}\|\bar{\omega}\|_{H^{1}}^{\tau} \leq \frac{c^{2}}{2}\|\bar{\omega}\|_{H^{1}}+C^{\prime}\|\bar{\omega}\|_{2},
$$

for some $C^{\prime}=C^{\prime}(M)$, it follows that

$$
4\|(\lambda-\mathbb{A}(f))[\bar{\omega}]\|_{H^{1}} \geq c\|\bar{\omega}\|_{H^{1}}-\frac{2 C^{\prime}}{c}\|\bar{\omega}\|_{2} .
$$

This estimate together with (3.19) leads us to (3.26) and the proof is complete.

We conclude this section by considering the adjoints of the operators defined in (3.2) and (3.5). Firstly we establish a similar estimate as in Theorem 3.5 for the operator $P(\mathbb{A}(f))^{*}$, where $(\mathbb{A}(f))^{*}$ is the double layer potential, cf. (3.3), and where $P: L_{2}(\mathbb{S}) \rightarrow \widehat{L}_{2}(\mathbb{S})$, with $P h:=h-\langle h\rangle$, denotes the orthogonal projection on $\widehat{L}_{2}(\mathbb{S})$. This estimate is important later on in the uniqueness proof of Theorem 1.1. Recalling that $(\mathbb{A}(f))^{*} \in \mathcal{L}\left(L_{2}(\mathbb{S})\right)$ is the $L_{2}$-adjoint of $\mathbb{A}(f) \in \mathcal{L}\left(L_{2}(\mathbb{S})\right)$, we obtain for $\bar{\omega}, \xi \in \widehat{L}_{2}(\mathbb{S})$ that

$$
\langle\mathbb{A}(f)[\bar{\omega}], \xi\rangle_{2}=\left\langle\bar{\omega},(\mathbb{A}(f))^{*}[\xi]\right\rangle_{2}=\left\langle\bar{\omega}, P(\mathbb{A}(f))^{*}[\xi]\right\rangle_{2},
$$

meaning that the adjoint $(\widehat{\mathbb{A}(f)})^{*}:=\left(\left.\mathbb{A}(f)\right|_{\widehat{L}_{2}(\mathbb{S})}\right)^{*} \in \mathcal{L}\left(\widehat{L}_{2}(\mathbb{S})\right)$ is given by $(\widehat{\mathbb{A}(f)})^{*}=P(\mathbb{A}(f))^{*}$.

${ }^{4}$ Letting $[\cdot, \cdot]_{\theta}$ denote complex interpolation functor, it is well-known that

$$
\left[H^{s_{0}}(\mathbb{S}), H^{s_{1}}(\mathbb{S})\right]_{\theta}=H^{(1-\theta) s_{0}+\theta s_{1}}(\mathbb{S}), \quad \theta \in(0,1),-\infty<s_{0} \leq s_{1}<\infty .
$$


Theorem 3.6. Let $M>0$. Then, there exists a constant $C=C(M)>0$ such that

$$
\|\xi\|_{H^{1}} \leq C\left\|\left(\lambda-(\widehat{\mathbb{A}(f)})^{*}\right)[\xi]\right\|_{H^{1}}
$$

for all $\lambda \in \mathbb{R}$ with $|\lambda| \geq 1, \xi \in \widehat{H}^{1}(\mathbb{S})$, and $f \in H^{2}(\mathbb{S})$ with $\|f\|_{H^{2}} \leq M$.

In particular, $\{\lambda \in \mathbb{R}:|\lambda| \geq 1\}$ is contained in the resolvent set of $(\widehat{\mathbb{A}(f)})^{*} \in \mathcal{L}\left(\widehat{H}^{1}(\mathbb{S})\right)$ for each $f \in H^{2}(\mathbb{S})$.

Proof. Let $M>0$. Taking advantage of the fact that $\lambda-(\widehat{\mathbb{A}(f)})^{*}$ is the $\mathcal{L}\left(\widehat{L}_{2}(\mathbb{S})\right)$-adjoint of $\lambda-\mathbb{A}(f)$ for each $\lambda \in \mathbb{R}$ and $f \in H^{r}(\mathbb{R}), r>3 / 2$, it follows from (3.19) there exists a constant $C=C(M)$ such that

$$
\|\xi\|_{2} \leq C\left\|\left(\lambda-(\widehat{\mathbb{A}(f)})^{*}\right)[\xi]\right\|_{2}
$$

for all $\lambda \in \mathbb{R}$ with $|\lambda| \geq 1, \xi \in \widehat{H}^{1}(\mathbb{S})$, and $f \in H^{2}(\mathbb{S})$ with $\|f\|_{H^{2}} \leq M$. In order to show that $(\widehat{\mathbb{A}(f)})^{*}[\xi] \in \widehat{H}^{1}(\mathbb{S})$, we note that

$$
(\widehat{\mathbb{A}(f)})^{*}[\xi]=(\mathbb{A}(f))^{*}[\xi]-\left\langle(\mathbb{A}(f))^{*}[\xi]\right\rangle=\mathbb{B}_{1}(f)[\xi]+\mathbb{B}_{2}(f)\left[f^{\prime} \xi\right]+\mathbb{A}_{3, *}(f)[\xi]-\left\langle(\mathbb{A}(f))^{*}[\xi]\right\rangle,
$$

where $\mathbb{B}_{1}(f)$ and $\mathbb{B}_{2}(f)$ are introduced in the proof of Lemma 3.1 and where

$$
\pi \mathbb{A}_{3, *}(f)[\xi]:=C_{1,1}(f)[f, \xi]-C_{0,1}(f)\left[f^{\prime} \xi\right] .
$$

The arguments used to derive (3.12) show that $\mathbb{A}_{3, *}(f)[\xi] \in H^{1}(\mathbb{S})$ with

$$
\begin{aligned}
\pi\left(\mathbb{A}_{3, *}(f)[\xi]\right)^{\prime}= & \pi \mathbb{A}_{3, *}(f)\left[\xi^{\prime}\right]+C_{1,1}(f)\left[f^{\prime}, \xi\right]-C_{0,1}(f)\left[f^{\prime \prime} \xi\right]+2 C_{2,2}(f, f)\left[f^{\prime}, f, f^{\prime} \xi\right] \\
& -2 C_{3,2}(f, f)\left[f^{\prime}, f, f, \xi\right],
\end{aligned}
$$

and together with $(3.9),(3.10)$ we conclude that indeed $(\widehat{\mathbb{A}(f)})^{*}[\xi] \in \widehat{H}^{1}(\mathbb{S})$. Proceeding as in Theorem 3.5, we may write

$$
\left((\widehat{\mathbb{A}(f)})^{*}[\xi]\right)^{\prime}=(\mathbb{A}(f))^{*}\left[\xi^{\prime}\right]+T_{\mathrm{lot}}^{A^{*}}(f)[\xi]=(\widehat{\mathbb{A}(f)})^{*}\left[\xi^{\prime}\right]+\left\langle(\mathbb{A}(f))^{*}\left[\xi^{\prime}\right]\right\rangle+T_{\mathrm{lot}}^{A^{*}}(f)[\xi],
$$

with

$$
\begin{aligned}
\pi T_{\mathrm{lot}}^{A^{*}}(f)[\xi]:= & C_{1,1}(f)\left[f^{\prime}, \xi\right]-C_{0,1}(f)\left[f^{\prime \prime} \xi\right]+2 C_{2,2}(f, f)\left[f^{\prime}, f, f^{\prime} \xi\right] \\
& -2 C_{3,2}(f, f)\left[f^{\prime}, f, f, \xi\right]+\pi\left(\left(\mathbb{B}_{2}(f)\left[f^{\prime} \xi\right]\right)^{\prime}-\mathbb{B}_{2}(f)\left[\left(f^{\prime} \xi\right)^{\prime}\right]\right) \\
& +\pi\left(\left(\mathbb{B}_{1}(f)[\xi]\right)^{\prime}-\mathbb{B}_{1}(f)\left[\xi^{\prime}\right]\right)
\end{aligned}
$$

satisfying

$$
\left\|T_{\mathrm{lot}}^{A^{*}}(f)[\xi]\right\|_{2} \leq C\|\xi\|_{H^{\tau}} \quad \text { for all } \xi \in \widehat{H}^{1}(\mathbb{S}),
$$

for any fixed $\tau \in(1 / 2,1)$ and with a constant $C=C(M)$. Moreover, since $\mathbb{A}(f)[1] \in H^{1}(\mathbb{S})$, it follows that

$$
\begin{aligned}
\left|\left\langle(\mathbb{A}(f))^{*}\left[\xi^{\prime}\right]\right\rangle\right| & \leq\left|\left\langle(\mathbb{A}(f))^{*}\left[\xi^{\prime}\right], 1\right\rangle_{2}\right|=\left|\left\langle\xi^{\prime}, \mathbb{A}(f)[1]\right\rangle_{2}\right|=\left|\int_{-\pi}^{\pi} \xi^{\prime} \mathbb{A}(f)[1] \mathrm{d} x\right| \\
& =\left|\int_{-\pi}^{\pi} \xi(\mathbb{A}(f)[1])^{\prime} \mathrm{d} x\right| \leq\|\xi\|_{2}\|\mathbb{A}(f)[1]\|_{H^{1}} \leq C\|\xi\|_{2},
\end{aligned}
$$

again with $C=C(M)$. The desired claim (3.31) follows now from (3.32), (3.33), and (3.34) by arguing as in Theorem 3.5.

Finally, given $f \in H^{r}(\mathbb{S}), r>3 / 2$, let $(\mathbb{B}(f))^{*} \in \mathcal{L}\left(L_{2}(\mathbb{S})\right)$ denote the adjoint of $\mathbb{B}(f) \in \mathcal{L}\left(L_{2}(\mathbb{S})\right)$. The next lemma is also used later on in the uniqueness proof of Theorem 1.1.

Lemma 3.7. Given $M>0$, there exists a constant $C=C(M)$ such that for all $f \in H^{2}(\mathbb{S})$ with $\|f\|_{H^{2}} \leq$ $M$ it holds that $(\mathbb{B}(f))^{*} \in \mathcal{L}\left(H^{1}(\mathbb{S})\right)$ and

$$
\left\|(\mathbb{B}(f))^{*}\right\|_{\mathcal{L}\left(H^{1}(\mathbb{S})\right)} \leq C .
$$


Proof. Given $f \in H^{2}(\mathbb{S})$, it is not difficult to show that

$$
(\mathbb{B}(f))^{*}[\xi]=-\mathbb{B}_{1}(f)\left[f^{\prime} \xi\right]+\mathbb{B}_{2}(f)[\xi]-\frac{1}{\pi}\left(C_{0,1}(f)[\xi]+C_{1,1}(f)\left[f, f^{\prime} \xi\right]\right), \quad \xi \in L_{2}(\mathbb{S}) .
$$

The desired estimate follows now by arguing as in Lemma 3.1.

\section{The Muskat Problem with Surface Tension Effects}

In this section we study the Muskat problem in the case when surface tension effects are included, that is for $\sigma>0$. The main goal of this section is to prove Theorem 1.1 which is postponed to the end of the section. As a first step we shall take advantage of the results established in the previous sections to reexpress the contour integral formulation (1.1) as an abstract evolution equation of the form

$$
\dot{f}(t)=\Phi_{\sigma}(f(t))[f(t)], \quad t>0, \quad f(0)=f_{0},
$$

with an operator $\left[f \mapsto \Phi_{\sigma}(f)\right]: H^{2}(\mathbb{S}) \rightarrow \mathcal{L}\left(H^{3}(\mathbb{S}), L_{2}(\mathbb{S})\right)$ defined in (4.7). The quasilinear character of the contour integral equation for $\sigma>0$ - which is not obvious because of the coupling in (1.1a) $)_{2}$-is expressed in (4.1) by the fact that $\Phi_{\sigma}$ is nonlinear with respect to the first variable $f \in H^{2}(\mathbb{S})$, but is linear with respect to the second variable $f \in H^{3}(\mathbb{S})$ which corresponds to the third spatial derivatives of the function $f=f(t, x)$ in the curvature term in (1.1a) $)_{2}$. A central part of the analysis in this section is devoted to showing that (4.1) is a parabolic problem in the sense that $\Phi_{\sigma}(f)$-viewed as an unbounded operator on $L_{2}(\mathbb{S})$ with definition domain $H^{3}(\mathbb{S})$ - is, for each $f \in H^{2}(\mathbb{S})$, the generator of a strongly continuous and analytic semigroup in $\mathcal{L}\left(L_{2}(\mathbb{S})\right)$, which we denote by writing

$$
-\Phi_{\sigma}(f) \in \mathcal{H}\left(H^{3}(\mathbb{S}), L_{2}(\mathbb{S})\right) .
$$

This property needs to be verified before applying the abstract quasilinear parabolic theory outlined in [1-5] (see also [50]) in the particular context of (4.1).

We begin by solving the Eq. (1.1a) $)_{2}$ for $\bar{\omega}$. We shall rely on the invertibility properties provided in Theorems 3.3 and 3.5 and the fact that the Atwood number satisfies $\left|a_{\mu}\right|<1$. In order to disclose the quasilinear structure of the Muskat problem with surface tension we address at this point the solvability of the equation

$$
\left(1+a_{\mu} \mathbb{A}(f)\right)[\bar{\omega}]=b_{\mu}\left[\sigma \frac{h^{\prime \prime \prime}}{\left(1+f^{\prime 2}\right)^{3 / 2}}-3 \sigma \frac{f^{\prime} f^{\prime \prime} h^{\prime \prime}}{\left(1+f^{\prime 2}\right)^{3 / 2}}-\Theta h^{\prime}\right]
$$

which for $h=f$ coincides, up to a factor of 2 , with $(1.1 \mathrm{a})_{2}$. The quasilinearity of the curvature term is essential here. For the sake of brevity we introduce

$$
b_{\mu}:=\frac{k}{\mu_{-}+\mu_{+}} .
$$

Since the values of $\sigma>0$ and $b_{\mu}>0$ are not important in the proof of Theorem 1.1 we set in this section

$$
b_{\mu}=\sigma=1 .
$$

The solvability result in Proposition 4.1 (a) below is the main step towards writing (1.1) in the form (4.1). The decomposition of the solution operator provided at Proposition $4.1(b)$ is essential later on in the proof of the generator property, as it enables us to use integration by parts when estimating some terms of leading order.

Proposition 4.1. (a) Given $f \in H^{2}(\mathbb{S})$ and $h \in H^{3}(\mathbb{S})$, the function

$$
\bar{\omega}(f)[h]:=\left(1+a_{\mu} \mathbb{A}(f)\right)^{-1}\left[\frac{h^{\prime \prime \prime}}{\left(1+f^{\prime 2}\right)^{3 / 2}}-3 \frac{f^{\prime} f^{\prime \prime} h^{\prime \prime}}{\left(1+f^{\prime 2}\right)^{3 / 2}}-\Theta h^{\prime}\right]
$$

is the unique solution to (4.3) in $\widehat{L}_{2}(\mathbb{S})$ and

$$
\bar{\omega} \in \mathrm{C}^{\omega}\left(H^{2}(\mathbb{S}), \mathcal{L}\left(H^{3}(\mathbb{S}), \widehat{L}_{2}(\mathbb{S})\right)\right) .
$$


(b) Given $f \in H^{2}(\mathbb{S})$ and $h \in H^{3}(\mathbb{S})$, let

$$
\begin{aligned}
& \bar{\omega}_{1}(f)[h]:=\left(1+a_{\mu} \mathbb{A}(f)\right)^{-1}\left[\frac{h^{\prime \prime}}{\left(1+f^{\prime 2}\right)^{3 / 2}}-\left\langle\frac{h^{\prime \prime}}{\left(1+f^{\prime 2}\right)^{3 / 2}}\right\rangle\right], \\
& \bar{\omega}_{2}(f)[h]:=\left(1+a_{\mu} \mathbb{A}(f)\right)^{-1}\left[-\Theta h^{\prime}+a_{\mu} T_{\operatorname{lot}}^{A}(f)\left[\bar{\omega}_{1}(f)[h]\right]\right],
\end{aligned}
$$

where $T_{\text {lot }}^{A}$ is defined in (3.28). Then:

(i) $\bar{\omega}_{1} \in \mathrm{C}^{\omega}\left(H^{2}(\mathbb{S}), \mathcal{L}\left(H^{3}(\mathbb{S}), \widehat{H}^{1}(\mathbb{S})\right)\right)$ and $\bar{\omega}_{2} \in \mathrm{C}^{\omega}\left(H^{2}(\mathbb{S}), \mathcal{L}\left(H^{3}(\mathbb{S}), \widehat{L}_{2}(\mathbb{S})\right)\right)$;

(ii)

$$
\bar{\omega}(f)=\frac{\mathrm{d}}{\mathrm{d} x} \circ \bar{\omega}_{1}(f)+\bar{\omega}_{2}(f)
$$

(iii) Given $\tau \in(1 / 2,1)$, there exists a constant $C$ such that

$$
\begin{aligned}
& \left\|\bar{\omega}_{1}(f)[h]\right\|_{2} \leq C\|h\|_{H^{2}} \\
& \left\|\bar{\omega}_{1}(f)[h]\right\|_{H^{\tau}}+\left\|\bar{\omega}_{2}(f)[h]\right\|_{2} \leq C\|h\|_{H^{2+\tau}} \quad \text { for all } h \in H^{3}(\mathbb{S}) .
\end{aligned}
$$

Proof. Observing that the right hand side of (4.3) belongs to $\widehat{L}_{2}(\mathbb{S})$, the claim $(a)$ follows from Theorems 3.3.

In order to prove $(b)$ we first note that

$$
\left[f \mapsto\left[h \mapsto \frac{h^{\prime \prime}}{\left(1+f^{\prime 2}\right)^{3 / 2}}-\left\langle\frac{h^{\prime \prime}}{\left(1+f^{\prime 2}\right)^{3 / 2}}\right\rangle\right]\right] \in \mathrm{C}^{\omega}\left(H^{2}(\mathbb{S}), \mathcal{L}\left(H^{3}(\mathbb{S}), \widehat{H}^{1}(\mathbb{S})\right)\right),
$$

and since by Theorem $3.5\left[f \mapsto\left(1+a_{\mu} \mathbb{A}(f)\right)^{-1}\right] \in \mathrm{C}^{\omega}\left(H^{2}(\mathbb{S}), \mathcal{L}\left(\widehat{H}^{1}(\mathbb{S})\right)\right)$, we conclude that $\bar{\omega}_{1}$ is welldefined together with $\bar{\omega}_{1} \in \mathrm{C}^{\omega}\left(H^{2}(\mathbb{S}), \mathcal{L}\left(H^{3}(\mathbb{S}), \widehat{H}^{1}(\mathbb{S})\right)\right)$. Recalling (3.27) and (3.28), it holds

$$
\left(1+a_{\mu} \mathbb{A}(f)\right)\left[\bar{\omega}(f)[h]-\left(\bar{\omega}_{1}(f)[h]\right)^{\prime}\right]=-\Theta h^{\prime}+a_{\mu} T_{\text {lot }}^{A}(f)\left[\bar{\omega}_{1}(f)[h]\right]=\left(1+a_{\mu} \mathbb{A}(f)\right)\left[\bar{\omega}_{2}(f)[h]\right] .
$$

This proves $\bar{\omega}_{2} \in \mathrm{C}^{\omega}\left(H^{2}(\mathbb{S}), \mathcal{L}\left(H^{3}(\mathbb{S}), \widehat{L}_{2}(\mathbb{S})\right)\right)$ together with the claim $(i i)$.

As for $($ iii), we note that the Theorems 3.3 and 3.5 imply that

$$
\left\|\bar{\omega}_{1}(f)[h]\right\|_{2} \leq C\|h\|_{H^{2}} \quad \text { and } \quad\left\|\bar{\omega}_{1}(f)[h]\right\|_{H^{1}} \leq C\|h\|_{H^{3}} \quad \text { for all } h \in H^{3}(\mathbb{S}),
$$

and the estimate $\left\|\bar{\omega}_{1}(f)[h]\right\|_{H^{\tau}} \leq C\|h\|_{H^{2+\tau}}, h \in H^{3}(\mathbb{S})$, follows from the latter via interpolation. Finally, recalling Theorem 3.3 and (3.29), it holds

$$
\begin{aligned}
\left\|\bar{\omega}_{2}(f)[h]\right\|_{2} & \leq C\left(\|h\|_{H^{1}}+\left\|T_{\mathrm{lot}}^{A}(f)\left[\bar{\omega}_{1}(f)[h]\right]\right\|_{2}\right) \leq C\left(\|h\|_{H^{1}}+\left\|\bar{\omega}_{1}(f)[h]\right\|_{H^{\tau}}\right) \\
& \leq C\|h\|_{H^{2+\tau}},
\end{aligned}
$$

and the proof is complete.

Proposition 4.1 enables us to recast the contour integral formulation (1.1) of the Muskat problem with surface tension as the abstract quasilinear evolution problem (4.1), where

$$
\Phi_{\sigma}(f)[h]:=\mathbb{B}(f)[\bar{\omega}(f)[h]] \quad \text { for } f \in H^{2}(\mathbb{S}) \text { and } h \in H^{3}(\mathbb{S}) .
$$

Proposition 4.1 and Lemma 3.1 imply that

$$
\Phi_{\sigma} \in \mathrm{C}^{\omega}\left(H^{2}(\mathbb{S}), \mathcal{L}\left(H^{3}(\mathbb{S}), \widehat{L}_{2}(\mathbb{S})\right)\right) \cap \mathrm{C}^{\omega}\left(H^{2}(\mathbb{S}), \mathcal{L}\left(H^{3}(\mathbb{S}), L_{2}(\mathbb{S})\right)\right)
$$

In the following $f \in H^{2}(\mathbb{S})$ is kept fixed. In order to establish the generator property (4.2) for $\Phi_{\sigma}(f)$ it is suitable to decompose this operator as the sum

$$
\Phi_{\sigma}(f)=\Phi_{\sigma, 1}(f)+\Phi_{\sigma, 2}(f),
$$

where

$$
\Phi_{\sigma, 1}(f)[h]=\mathbb{B}(f)\left[\left(\bar{\omega}_{1}(f)[h]\right)^{\prime}\right] \quad \text { and } \quad \Phi_{\sigma, 2}(f)[h]=\mathbb{B}(f)\left[\bar{\omega}_{2}(f)[h]\right] .
$$

The operator $\Phi_{\sigma, 1}(f)$ can be viewed as the leading order part of $\Phi_{\sigma}(f)$, while $\Phi_{\sigma, 2}(f)$ is a lower order perturbation, see the proof of Theorem 4.3. We study first the leading order part $\Phi_{\sigma, 1}(f)$. In order 
to establish (4.2) we follow a direct and self-contained approach pursued previously in $[29,33,35]$ and generalized more recently in $[32,47-49]$ in the context of the Muskat problem. The proof of $(4.2)$ uses a localization procedure which necessitates the introduction of certain partitions of unity for the unit circle.

To proceed, we choose for each integer $p \geq 3$ a set $\left\{\pi_{j}^{p}: 1 \leq j \leq 2^{p+1}\right\} \subset \mathrm{C}^{\infty}(\mathbb{S},[0,1])$, called p-partition of unity, such that

- $\quad \operatorname{supp} \pi_{j}^{p}=\bigcup_{n \in \mathbb{Z}}\left(2 \pi n+I_{j}^{p}\right)$ and $I_{j}^{p}:=[j-5 / 3, j-1 / 3] \frac{\pi}{2^{p}} ;$

- $\sum_{j=1}^{2^{p+1}} \pi_{j}^{p}=1$ in $\mathrm{C}(\mathbb{S})$.

To each such $p$-partition of unity we associate a set $\left\{\chi_{j}^{p}: 1 \leq j \leq 2^{p+1}\right\} \subset \mathrm{C}^{\infty}(\mathbb{S},[0,1])$ satisfying

$$
\begin{aligned}
& \text { - } \operatorname{supp} \chi_{j}^{p}=\bigcup_{n \in \mathbb{Z}}\left(2 \pi n+J_{j}^{p}\right) \text { with } I_{j}^{p} \subset J_{j}^{p}:=[j-8 / 3, j+2 / 3] \frac{\pi}{2^{p}} ; \\
& \text { - } \chi_{j}^{p}=1 \text { on } I_{j}^{p} .
\end{aligned}
$$

As a further step we introduce the continuous path

$$
\left[\tau \mapsto \Phi_{\sigma, 1}(\tau f)\right]:[0,1] \rightarrow \mathcal{L}\left(H^{3}(\mathbb{S}), L_{2}(\mathbb{S})\right),
$$

which connects the operator $\Phi_{\sigma, 1}(f)$ with the Fourier multiplier

$$
\Phi_{\sigma, 1}(0)[h](x)=\mathbb{B}(0)\left[h^{\prime \prime \prime}\right](x)=\frac{1}{2 \pi} \mathrm{PV} \int_{-\pi}^{\pi} \frac{h^{\prime \prime \prime}(x-s)}{t_{[s]}} \mathrm{d} s=H\left[h^{\prime \prime \prime}\right](x),
$$

where $H$ denotes as usually the periodic Hilbert transform. Since $H$ is the Fourier multiplier with symbol $(-i \operatorname{sign}(k))_{k \in \mathbb{Z}}$, it follows that $\Phi_{\sigma, 1}(0)=-\left(\partial_{x}^{4}\right)^{3 / 4}$, that is the symbol of $\Phi_{\sigma, 1}(0)$ is $\left(-|k|^{3}\right)_{k \in \mathbb{Z}}$. In Theorem 4.2, which is the key argument in the proof of (4.2), we establish some commutator type estimates relating $\Phi_{\sigma, 1}(\tau f)$ locally to some explicit Fourier multipliers. The proof of this result is quite technical and lengthy and uses to a large extent the outcome of Lemma A.1.

Theorem 4.2. Let $f \in H^{2}(\mathbb{S})$ and $\mu>0$ be given. Then, there exist $p \geq 3$, a p-partition of unity $\left\{\pi_{j}^{p}\right.$ : $\left.1 \leq j \leq 2^{p+1}\right\}$, a constant $K=K(p)$, and for each $j \in\left\{1, \ldots, 2^{p+1}\right\}$ and $\tau \in[0,1]$ there exist operators

$$
\mathbb{A}_{j, \tau} \in \mathcal{L}\left(H^{3}(\mathbb{S}), L_{2}(\mathbb{S})\right)
$$

such that

$$
\left\|\pi_{j}^{p} \Phi_{\sigma, 1}(\tau f)[h]-\mathbb{A}_{j, \tau}\left[\pi_{j}^{p} h\right]\right\|_{2} \leq \mu\left\|\pi_{j}^{p} h\right\|_{H^{3}}+K\|h\|_{H^{11 / 4}}
$$

for all $j \in\left\{1, \ldots, 2^{p+1}\right\}, \tau \in[0,1]$, and $h \in H^{3}(\mathbb{S})$. The operator $\mathbb{A}_{j, \tau}$ is defined by

$$
\mathbb{A}_{j, \tau}:=-\frac{1}{\left(1+f_{\tau}^{\prime 2}\left(x_{j}^{p}\right)\right)^{3 / 2}}\left(\partial_{x}^{4}\right)^{3 / 4}
$$

where $x_{j}^{p} \in I_{j}^{p}$ is arbitrary, but fixed.

Proof. Let $p \geq 3$ be an integer which we fix later on in this proof and let $\left\{\pi_{j}^{p}: 1 \leq j \leq 2^{p+1}\right\}$ be a $p$-partition of unity, respectively, let $\left\{\chi_{j}^{p}: 1 \leq j \leq 2^{p+1}\right\}$ be a family associated to this $p$-partition of unity as described above. In the following, we denote by $C$ constants which are independent of $p \in \mathbb{N}$, $h \in H^{3}(\mathbb{S}), \tau \in[0,1]$, and $j \in\left\{1, \ldots, 2^{p+1}\right\}$, while the constants denoted by $K$ may depend only on $p$.

Step 1: The lower order terms. Using the decomposition provided in the proof of Lemma 3.1 for the operator $\mathbb{B}$, we write

$$
\Phi_{\sigma, 1}(\tau f)[h]=f_{\tau}^{\prime} \mathbb{B}_{1}\left(f_{\tau}\right)\left[\bar{\omega}_{1}^{\prime}\right]-\mathbb{B}_{2}\left(f_{\tau}\right)\left[\bar{\omega}_{1}^{\prime}\right]+\frac{1}{\pi} C_{0,1}\left(f_{\tau}\right)\left[\bar{\omega}_{1}^{\prime}\right]+\frac{1}{\pi} f_{\tau}^{\prime} C_{1,1}\left(f_{\tau}\right)\left[f_{\tau}, \bar{\omega}_{1}^{\prime}\right],
$$

where, for the sake of brevity, we have set

$$
\bar{\omega}_{1}:=\bar{\omega}_{1}(\tau f)[h] \quad \text { and } \quad f_{\tau}:=\tau f .
$$


Using integration by parts, we infer from (4.6) that

$$
\left\|\pi_{j}^{p}\left[f_{\tau}^{\prime} \mathbb{B}_{1}\left(f_{\tau}\right)\left[\bar{\omega}_{1}^{\prime}\right]-\mathbb{B}_{2}\left(f_{\tau}\right)\left[\bar{\omega}_{1}^{\prime}\right]\right]\right\|_{2} \leq C\left\|\bar{\omega}_{1}\right\|_{2} \leq C\|h\|_{H^{2}},
$$

and we are left to consider the last two terms in (4.11).

Step 2: The first leading order term. Given $1 \leq j \leq 2^{p+1}$ and $\tau \in[0,1]$, let

$$
\mathbb{A}_{j, \tau}^{1}:=-\frac{f_{\tau}^{\prime 2}\left(x_{j}^{p}\right)}{\left(1+f_{\tau}^{\prime 2}\left(x_{j}^{p}\right)\right)^{5 / 2}}\left(\partial_{x}^{4}\right)^{3 / 4},
$$

where $x_{j}^{p} \in I_{j}^{p}$. In this step we show that if $p$ is sufficiently large, then

$$
\left\|\pi_{j}^{p} f_{\tau}^{\prime} C_{1,1}\left(f_{\tau}\right)\left[f_{\tau}, \bar{\omega}_{1}^{\prime}\right]-\left.\pi \mathbb{A}_{j, \tau}^{1}\left[\pi_{j}^{p} h\right]\right|_{2} \leq \frac{\mu}{2}\right\| \pi_{j}^{p} h\left\|_{H^{3}}+K\right\| h \|_{H^{11 / 4}}
$$

for all $j \in\left\{1, \ldots, 2^{p+1}\right\}, \tau \in[0,1]$, and $h \in H^{3}(\mathbb{S})$. To this end we write

$$
\pi_{j}^{p} f_{\tau}^{\prime} C_{1,1}\left(f_{\tau}\right)\left[f_{\tau}, \bar{\omega}_{1}^{\prime}\right]-\pi \mathbb{A}_{j, \tau}^{1}\left[\pi_{j}^{p} h\right]=T_{1}[h]+T_{2}[h]+T_{3}[h],
$$

where

$$
\begin{aligned}
& T_{1}[h]:=\pi_{j}^{p} f_{\tau}^{\prime} C_{1,1}\left(f_{\tau}\right)\left[f_{\tau}, \bar{\omega}_{1}^{\prime}\right]-f_{\tau}^{\prime}\left(x_{j}^{p}\right) C_{1,1}\left(f_{\tau}\right)\left[f_{\tau}, \pi_{j}^{p} \bar{\omega}_{1}^{\prime}\right], \\
& T_{2}[h]:=f_{\tau}^{\prime}\left(x_{j}^{p}\right) C_{1,1}\left(f_{\tau}\right)\left[f_{\tau}, \pi_{j}^{p} \bar{\omega}_{1}^{\prime}\right]-\frac{f_{\tau}^{\prime 2}\left(x_{j}^{p}\right)}{1+f_{\tau}^{\prime 2}\left(x_{j}^{p}\right)} C_{0,0}\left[\pi_{j}^{p} \bar{\omega}_{1}^{\prime}\right], \\
& T_{3}[h]:=\frac{f_{\tau}^{\prime 2}\left(x_{j}^{p}\right)}{1+f_{\tau}^{\prime 2}\left(x_{j}^{p}\right)}\left[C_{0,0}\left[\pi_{j}^{p} \bar{\omega}_{1}^{\prime}\right]+\frac{\pi}{\left(1+f_{\tau}^{\prime 2}\left(x_{j}^{p}\right)\right)^{3 / 2}}\left(\partial_{x}^{4}\right)^{3 / 4}\left[\pi_{j}^{p} h\right]\right] .
\end{aligned}
$$

We first consider $T_{1}[h]$. Recalling that $\chi_{j}^{p} \pi_{j}^{p}=\pi_{j}^{p}$, algebraic manipulations lead us to

$$
T_{1}[h]:=\chi_{j}^{p}\left(f_{\tau}^{\prime}-f_{\tau}^{\prime}\left(x_{j}^{p}\right)\right) C_{1,1}\left(f_{\tau}\right)\left[f_{\tau}, \pi_{j}^{p} \bar{\omega}_{1}^{\prime}\right]+T_{11}[h],
$$

and the term $T_{11}[h]$ may be expressed, after integrating by parts, as

$$
\begin{aligned}
T_{11}[h]= & f_{\tau}^{\prime} C_{1,1}\left(f_{\tau}\right)\left[f_{\tau},\left(\pi_{j}^{p}\right)^{\prime} \bar{\omega}_{1}\right]-2 f_{\tau}^{\prime} C_{2,1}\left(f_{\tau}\right)\left[\pi_{j}^{p}, f_{\tau}, \bar{\omega}_{1}\right]+f_{\tau}^{\prime} C_{1,1}\left(f_{\tau}\right)\left[\pi_{j}^{p}, f_{\tau}^{\prime} \bar{\omega}_{1}\right] \\
& -2 f_{\tau}^{\prime} C_{3,2}\left(f_{\tau}, f_{\tau}\right)\left[\pi_{j}^{p}, f_{\tau}, f_{\tau}, f_{\tau}^{\prime} \bar{\omega}_{1}\right]+2 f_{\tau}^{\prime} C_{4,2}\left(f_{\tau}, f_{\tau}\right)\left[\pi_{j}^{p}, f_{\tau}, f_{\tau}, f_{\tau}, \bar{\omega}_{1}\right] \\
& +\left(f_{\tau}^{\prime}\left(x_{j}^{p}\right)-f_{\tau}^{\prime}\right)\left(1-\chi_{j}^{p}\right) C_{1,1}\left(f_{\tau}\right)\left[f_{\tau},\left(\pi_{j}^{p}\right)^{\prime} \bar{\omega}_{1}\right] \\
& +\left(f_{\tau}^{\prime}\left(x_{j}^{p}\right)-f_{\tau}^{\prime}\right) C_{1,1}\left(f_{\tau}\right)\left[\chi_{j}^{p}, \pi_{j}^{p} f_{\tau}^{\prime} \bar{\omega}_{1}\right]-2\left(f_{\tau}^{\prime}\left(x_{j}^{p}\right)-f_{\tau}^{\prime}\right) C_{2,1}\left(f_{\tau}\right)\left[\chi_{j}^{p}, f_{\tau}, \pi_{j}^{p} \bar{\omega}_{1}\right] \\
& -2\left(f_{\tau}^{\prime}\left(x_{j}^{p}\right)-f_{\tau}^{\prime}\right) C_{3,2}\left(f_{\tau}, f_{\tau}\right)\left[\chi_{j}^{p}, f_{\tau}, f_{\tau}, \pi_{j}^{p} f_{\tau}^{\prime} \bar{\omega}_{1}\right] \\
& +2\left(f_{\tau}^{\prime}\left(x_{j}^{p}\right)-f_{\tau}^{\prime}\right) C_{4,2}\left(f_{\tau}, f_{\tau}\right)\left[\chi_{j}^{p}, f_{\tau}, f_{\tau}, f_{\tau}, \pi_{j}^{p} \bar{\omega}_{1}\right] .
\end{aligned}
$$

Lemma A.1 ( $i$ ) together with (4.6) yields

$$
\left\|T_{11}[h]\right\|_{2} \leq K\left\|\bar{\omega}_{1}\right\|_{2} \leq K\|h\|_{H^{2}},
$$

and

$$
\left\|C_{1,1}\left(f_{\tau}\right)\left[f_{\tau}, \pi_{j}^{p} \bar{\omega}_{1}^{\prime}\right]\right\|_{2} \leq C\left\|\pi_{j}^{p} \bar{\omega}_{1}^{\prime}\right\|_{2} .
$$

Hence, we need to estimate the term $\left\|\pi_{j}^{p} \bar{\omega}_{1}^{\prime}\right\|_{2}$ appropriately. The relation (3.27) and the definition of $\bar{\omega}_{1}$ (see Proposition $4.1(b)$ ), yield

$$
\begin{aligned}
\left(1+a_{\mu} \mathbb{A}\left(f_{\tau}\right)\right)\left[\left(\pi_{j}^{p} \bar{\omega}_{1}\right)^{\prime}\right]= & \frac{\pi_{j}^{p} h^{\prime \prime \prime}}{\left(1+f_{\tau}^{\prime 2}\right)^{3 / 2}}-\frac{3 \pi_{j}^{p} f_{\tau}^{\prime} f_{\tau}^{\prime \prime} h^{\prime \prime}}{\left(1+f_{\tau}^{\prime 2}\right)^{5 / 2}}-a_{\mu} \pi_{j}^{p} T_{\mathrm{lot}}^{A}\left(f_{\tau}\right)\left[\bar{\omega}_{1}\right] \\
& +\left(1+a_{\mu} \mathbb{A}\left(f_{\tau}\right)\right)\left[\left(\pi_{j}^{p}\right)^{\prime} \bar{\omega}_{1}\right]+a_{\mu}\left(\mathbb{A}\left(f_{\tau}\right)\left[\pi_{j}^{p} \bar{\omega}_{1}^{\prime}\right]-\pi_{j}^{p} \mathbb{A}\left(f_{\tau}\right)\left[\bar{\omega}_{1}^{\prime}\right]\right),
\end{aligned}
$$

and the last term on the right hand side of (4.16) can be recast as

$$
\begin{aligned}
\pi\left(\mathbb{A}\left(f_{\tau}\right)\left[\pi_{j}^{p} \bar{\omega}_{1}^{\prime}\right]-\pi_{j}^{p} \mathbb{A}\left(f_{\tau}\right)\left[\bar{\omega}_{1}^{\prime}\right]\right)= & \pi f_{\tau}^{\prime}\left(\pi_{j}^{p} \mathbb{B}_{2}\left(f_{\tau}\right)\left[\bar{\omega}_{1}^{\prime}\right]-\mathbb{B}_{2}\left(f_{\tau}\right)\left[\pi_{j}^{p} \bar{\omega}_{1}^{\prime}\right]\right) \\
& +\pi\left(\pi_{j}^{p} \mathbb{B}_{1}\left(f_{\tau}\right)\left[\bar{\omega}_{1}^{\prime}\right]-\mathbb{B}_{1}\left(f_{\tau}\right)\left[\pi_{j}^{p} \bar{\omega}_{1}^{\prime}\right]\right)
\end{aligned}
$$




$$
\begin{aligned}
& +f_{\tau}^{\prime}\left(C_{0,1}\left(f_{\tau}\right)\left[\pi_{j}^{p} \bar{\omega}_{1}^{\prime}\right]-\pi_{j}^{p} C_{0,1}\left(f_{\tau}\right)\left[\bar{\omega}_{1}^{\prime}\right]\right) \\
& -\left(C_{1,1}\left(f_{\tau}\right)\left[f_{\tau}, \pi_{j}^{p} \bar{\omega}_{1}^{\prime}\right]-\pi_{j}^{p} C_{1,1}\left(f_{\tau}\right)\left[f_{\tau}, \bar{\omega}_{1}^{\prime}\right]\right) .
\end{aligned}
$$

Integration by parts and Lemma A.1 ( $i$ ) lead us to

$$
\begin{aligned}
& \left\|f_{\tau}^{\prime} \mathbb{B}_{2}\left(f_{\tau}\right)\left[\pi_{j}^{p} \bar{\omega}_{1}^{\prime}\right]\right\|_{2}+\left\|f_{\tau}^{\prime} \pi_{j}^{p} \mathbb{B}_{2}\left(f_{\tau}\right)\left[\bar{\omega}_{1}^{\prime}\right]\right\|_{2}+\left\|\pi_{j}^{p} \mathbb{B}_{1}\left(f_{\tau}\right)\left[\bar{\omega}_{1}^{\prime}\right]\right\|_{2}+\left\|\mathbb{B}_{1}\left(f_{\tau}\right)\left[\pi_{j}^{p} \bar{\omega}_{1}^{\prime}\right]\right\|_{2} \\
& \quad+\left\|f_{\tau}^{\prime}\left(C_{0,1}\left(f_{\tau}\right)\left[\pi_{j}^{p} \bar{\omega}_{1}^{\prime}\right]-\pi_{j}^{p} C_{0,1}\left(f_{\tau}\right)\left[\bar{\omega}_{1}^{\prime}\right]\right)\right\|_{2}+\left\|C_{1,1}\left(f_{\tau}\right)\left[f_{\tau}, \pi_{j}^{p} \bar{\omega}_{1}^{\prime}\right]-\pi_{j}^{p} C_{1,1}\left(f_{\tau}\right)\left[f_{\tau}, \bar{\omega}_{1}^{\prime}\right]\right\|_{2} \\
& \quad \leq K\left\|\bar{\omega}_{1}\right\|_{2} \leq K\|h\|_{H^{2}} .
\end{aligned}
$$

Theorem 3.3, Lemma 3.2 (which can be applied as $\left(\pi_{j}^{p} \bar{\omega}_{1}\right)^{\prime} \in \widehat{L}^{2}(\mathbb{S})$ ), (3.29) and (4.6) (both for $\tau=3 / 4$ ), and (4.16), (4.17) combined yield

$$
\left\|\left(\pi_{j}^{p} \bar{\omega}_{1}\right)^{\prime}\right\|_{2} \leq C\left\|\pi_{j}^{p} h\right\|_{H^{3}}+K\|h\|_{H^{11 / 12}}+K\left\|\bar{\omega}_{1}\right\|_{H^{3 / 4}} \leq C\left\|\pi_{j}^{p} h\right\|_{H^{3}}+K\|h\|_{H^{11 / 12}},
$$

and (4.6) now entails

$$
\left\|\pi_{j}^{p} \bar{\omega}_{1}^{\prime}\right\|_{2} \leq\left\|\left(\pi_{j}^{p} \bar{\omega}_{1}\right)^{\prime}\right\|_{2}+\left\|\left(\pi_{j}^{p}\right)^{\prime} \bar{\omega}_{1}\right\|_{2} \leq C\left\|\pi_{j}^{p} h\right\|_{H^{3}}+K\|h\|_{H^{11 / 12}} .
$$

Recalling that $x_{j}^{p} \in I_{j}^{p} \subset J_{j}^{p}$ and $\operatorname{supp} \chi_{j}^{p}=\cup_{n \in \mathbb{Z}}\left(2 \pi n+J_{j}^{p}\right)$, the embedding $H^{1}(\mathbb{S}) \hookrightarrow \mathrm{C}^{1 / 2}(\mathbb{S})$ together with (4.14) (4.15), and (4.18) finally yield

$$
\begin{aligned}
\left\|T_{1}[h]\right\|_{2} & \leq C\left\|\chi_{j}^{p}\left(f^{\prime}-f^{\prime}\left(x_{j}^{p}\right)\right)\right\|_{\infty}\left\|\pi_{j}^{p} h\right\|_{H^{3}}+K\|h\|_{H^{11 / 12}} \leq \frac{C}{2^{p / 2}}\left\|\pi_{j}^{p} h\right\|_{H^{3}}+K\|h\|_{H^{11 / 12}} \\
& \leq \frac{\mu}{6}\left\|\pi_{j}^{p} h\right\|_{H^{3}}+K\|h\|_{H^{11 / 12}},
\end{aligned}
$$

provided that $p$ is sufficiently large.

Noticing that

$$
\frac{f_{\tau}^{\prime}\left(x_{j}^{p}\right)}{1+f_{\tau}^{\prime 2}\left(x_{j}^{p}\right)} C_{0,0}\left[\pi_{j}^{p} \bar{\omega}_{1}^{\prime}\right]=C_{1,1}\left(f_{\tau}^{\prime}\left(x_{j}^{p}\right) \operatorname{id}_{\mathbb{R}}\right)\left[f_{\tau}^{\prime}\left(x_{j}^{p}\right) \operatorname{id}_{\mathbb{R}}, \pi_{j}^{p} \bar{\omega}_{1}^{\prime}\right]
$$

we write the term $T_{2}[h]$ as

$$
T_{2}[h]=f_{\tau}^{\prime}\left(x_{j}^{p}\right) T_{21}[h]-\frac{f_{\tau}^{\prime 2}\left(x_{j}^{p}\right)}{1+f_{\tau}^{\prime 2}\left(x_{j}^{p}\right)} T_{22}[h],
$$

where

$$
\begin{aligned}
& T_{21}[h]:=C_{1,1}\left(f_{\tau}\right)\left[f_{\tau}-f_{\tau}^{\prime}\left(x_{j}^{p}\right) \operatorname{id}_{\mathbb{R}}, \pi_{j}^{p} \bar{\omega}_{1}^{\prime}\right], \\
& T_{22}[h]:=C_{2,1}\left(f_{\tau}\right)\left[f_{\tau}-f_{\tau}^{\prime}\left(x_{j}^{p}\right) \operatorname{id}_{\mathbb{R}}, f_{\tau}+f_{\tau}^{\prime}\left(x_{j}^{p}\right) \operatorname{id}_{\mathbb{R}}, \pi_{j}^{p} \bar{\omega}_{1}^{\prime}\right] .
\end{aligned}
$$

Though $f_{\tau}^{\prime}\left(x_{j}^{p}\right) \operatorname{id}_{\mathbb{R}}$ is not $2 \pi$-periodic, it is easy to see that the functions $T_{2 i}[h]$ still belong to $L_{2}(\mathbb{S})$ for $i \in\{1,2\}$. Since $\chi_{j}^{p} \pi_{j}^{p}=\pi_{j}^{p}$, we have

$$
T_{21}[h]:=T_{21 a}[h]+T_{21 b}[h],
$$

where

$$
\begin{aligned}
T_{21 a}[h]:= & \chi_{j}^{p} \mathrm{PV} \int_{|s|<\frac{\pi}{2^{p}}} \frac{\delta_{[\cdot, s]}\left(f_{\tau}-f_{\tau}^{\prime}\left(x_{j}^{p}\right) \operatorname{id}_{\mathbb{R}}\right) / s}{1+\left(\delta_{[\cdot, s]} f_{\tau} / s\right)^{2}} \frac{\left(\pi_{j}^{p} \bar{\omega}_{1}^{\prime}\right)(\cdot-s)}{s} \mathrm{~d} s \\
T_{21 b}[h]:= & \chi_{j}^{p} \mathrm{PV} \int_{\frac{\pi}{2 p}<|s|<\pi} \frac{\delta_{[\cdot, s]}\left(f_{\tau}-f_{\tau}^{\prime}\left(x_{j}^{p}\right) \mathrm{id}_{\mathbb{R}}\right) / s}{1+\left(\delta_{[\cdot, s]} f_{\tau} / s\right)^{2}} \frac{\left(\pi_{j}^{p} \bar{\omega}_{1}^{\prime}\right)(\cdot-s)}{s} \mathrm{~d} s \\
& -\int_{-\pi}^{\pi} \frac{\left(\delta_{[\cdot, s]}\left(f_{\tau}-f_{\tau}^{\prime}\left(x_{j}^{p}\right) \mathrm{id}_{\mathbb{R}}\right) / s\right)\left(\delta_{[\cdot, s]} \chi_{j}^{p} / s\right)}{1+\left(\delta_{[\cdot, s]} f_{\tau} / s\right)^{2}}\left(\pi_{j}^{p} \bar{\omega}_{1}^{\prime}\right)(\cdot-s) \mathrm{d} s .
\end{aligned}
$$

Integrating by parts we obtain in view of (4.6) that

$$
\left\|T_{21 b}[h]\right\|_{2} \leq K\left\|\bar{\omega}_{1}\right\|_{2} \leq K\|h\|_{H^{2}} .
$$


Since $T_{21 a}[h] \in L_{2}(\mathbb{S})$, it holds $\left\|T_{21 a}[h]\right\|_{2}=\left\|T_{21 a}[h]\right\|_{L_{2}((-\pi, \pi))}$. Clearly, if $x \in \operatorname{supp}\left(\mathbf{1}_{(-\pi, \pi)} T_{21 a}[h]\right)$, then

$$
x \in(-\pi, \pi) \cap\left(\cup_{n \in \mathbb{Z}}\left(2 n \pi+J_{j}^{p}\right)\right) .
$$

Letting $J_{j}^{p}:=\left[a_{j}^{p}, b_{j}^{p}\right], p \geq 3,1 \leq j \leq 2^{p+1}$, we distinguish three cases.

(i) If $1 \leq j \leq 2^{p}-1$, then $(-\pi, \pi) \cap\left(2 \pi n+J_{j}^{p}\right) \neq \emptyset$ if and only if $n=0$ and

$$
(-\pi, \pi) \cap J_{j}^{p}=\left[a_{j}^{p}, b_{j}^{p}\right] .
$$

(ii) If $2^{p}+3 \leq j \leq 2^{p+1}$, then $(-\pi, \pi) \cap\left(2 \pi n+J_{j}^{p}\right) \neq \emptyset$ if and only if $n=-1$ and

$$
(-\pi, \pi) \cap\left(-2 \pi+J_{j}^{p}\right)=\left[a_{j}^{p}-2 \pi, b_{j}^{p}-2 \pi\right] .
$$

(iii) If $j \in\left\{2^{p}, 2^{p}+1,2^{p}+2\right\}$, then $(-\pi, \pi) \cap\left(2 \pi n+J_{j}^{p}\right) \neq \emptyset$ if and only if $n \in\{-1,0\}$, and

$$
(-\pi, \pi) \cap J_{j}^{p}=\left[a_{j}^{p}, \pi\right) \quad \text { and } \quad(-\pi, \pi) \cap\left(-2 \pi+J_{j}^{p}\right)=\left(-\pi,-2 \pi+b_{j}^{p}\right] .
$$

Assume that we are in the first case, that is $1 \leq j \leq 2^{p}-1$. Let $F_{\tau, j}$ be the Lipschitz continuous function given by

$$
F_{\tau, j}=f_{\tau} \quad \text { on }\left[a_{j}^{p}, b_{j}^{p}\right], \quad F_{\tau, j}^{\prime}=f_{\tau}^{\prime}\left(x_{j}^{p}\right) \quad \text { on } \mathbb{R} \backslash\left[a_{j}^{p}, b_{j}^{p}\right] .
$$

Then $\left\|F_{\tau, j}^{\prime}\right\|_{\infty} \leq\left\|f^{\prime}\right\|_{\infty}$. Taking into account that $\left(\operatorname{supp} \pi_{j}^{p}\right) \cap\left[a_{j}^{p}-\pi / 2^{p}, b_{j}^{p}+\pi / 2^{p}\right] \subset\left[a_{j}^{p}, b_{j}^{p}\right]$, it follows that

$$
\begin{aligned}
\mathbf{1}_{(-\pi, \pi)} T_{21 a}[h]= & \mathbf{1}_{(-\pi, \pi)} \chi_{j}^{p} \mathrm{PV} \int_{|s|<\frac{\pi}{2^{p}}} \frac{\delta_{[\cdot, s]}\left(F_{\tau, j}-f_{\tau}^{\prime}\left(x_{j}^{p}\right) \mathrm{id}_{\mathbb{R}}\right) / s}{1+\left(\delta_{[\cdot, s]} f_{\tau} / s\right)^{2}} \frac{\left(\pi_{j}^{p} \bar{\omega}_{1}^{\prime}\right)(\cdot-s)}{s} \mathrm{~d} s, \\
= & \mathbf{1}_{(-\pi, \pi)} \chi_{j}^{p} C_{1,1}\left(f_{\tau}\right)\left[F_{\tau, j}-f_{\tau}^{\prime}\left(x_{j}^{p}\right) \operatorname{id}_{\mathbb{R}}, \pi_{j}^{p} \bar{\omega}_{1}^{\prime}\right] \\
& -\mathbf{1}_{(-\pi, \pi)} \chi_{j}^{p} \mathrm{PV} \int_{\frac{\pi}{2^{p}}<|s|<\pi} \frac{\delta_{[\cdot, s]}\left(F_{\tau, j}-f_{\tau}^{\prime}\left(x_{j}^{p}\right) \mathrm{id}_{\mathbb{R}}\right) / s}{1+\left(\delta_{[\cdot, s]} f_{\tau} / s\right)^{2}} \frac{\left(\pi_{j}^{p} \bar{\omega}_{1}^{\prime}\right)(\cdot-s)}{s} \mathrm{~d} s,
\end{aligned}
$$

and, using integration by parts and (4.6), we arrive at

$$
\left\|\chi_{j}^{p} \mathrm{PV} \int_{\frac{\pi}{2^{p}}<|s|<\pi} \frac{\delta_{[\cdot, s]}\left(F_{\tau, j}-f_{\tau}^{\prime}\left(x_{j}^{p}\right) \operatorname{id}_{\mathbb{R}}\right) / s}{1+\left(\delta_{[\cdot, s]} f_{\tau} / s\right)^{2}} \frac{\left(\pi_{j}^{p} \bar{\omega}_{1}^{\prime}\right)(\cdot-s)}{s} \mathrm{~d} s\right\|_{L_{2}((-\pi, \pi))} \leq K\left\|\bar{\omega}_{1}\right\|_{2} \leq K\|h\|_{H^{2}} .
$$

Moreover, combining Lemma A.1 (i) and (4.18), we find that

$$
\begin{aligned}
\left\|\chi_{j}^{p} C_{1,1}\left(f_{\tau}\right)\left[F_{\tau, j}-f_{\tau}^{\prime}\left(x_{j}^{p}\right) \operatorname{id}_{\mathbb{R}}, \pi_{j}^{p} \bar{\omega}_{1}^{\prime}\right]\right\|_{L_{2}((-\pi, \pi))} & \leq C\left\|F_{\tau, j}^{\prime}-f_{\tau}^{\prime}\left(x_{j}^{p}\right)\right\|_{\infty}\left\|\pi_{j}^{p} \bar{\omega}_{1}^{\prime}\right\|_{2} \\
& \leq C\left\|f^{\prime}-f^{\prime}\left(x_{j}^{p}\right)\right\|_{L_{\infty}\left(\left(a_{j}^{p}, b_{j}^{p}\right)\right)}\left(\left\|\pi_{j}^{p} h\right\|_{H^{3}}+K\|h\|_{H^{11 / 12}}\right) \\
& \leq \frac{\mu}{12}\left\|\pi_{j}^{p} h\right\|_{H^{3}}+K\|h\|_{H^{11 / 12}},
\end{aligned}
$$

provided that $p$ is sufficiently large. Altogether, we conclude that for $1 \leq j \leq 2^{p}-1$ it holds

$$
\left\|T_{21}[h]\right\|_{2} \leq \frac{\mu}{12}\left\|\pi_{j}^{p} h\right\|_{H^{3}}+K\|h\|_{H^{11 / 12}} .
$$

Similar arguments apply also in the cases $($ ii $)$ and (iii), and therefore the latter estimate actually holds for all $1 \leq j \leq 2^{p+1}$. Since $T_{22}[h]$ can be estimated in the same way, we obtain that

$$
\left\|T_{2}[h]\right\|_{2} \leq \frac{\mu}{6}\left\|\pi_{j}^{p} h\right\|_{H^{3}}+K\|h\|_{H^{11 / 12}},
$$

provided that $p$ is sufficiently large.

With regard to $T_{3}[h]$, it holds

$$
\left\|T_{3}[h]\right\|_{2} \leq\left\|C_{0,0}\left[\pi_{j}^{p} \bar{\omega}_{1}^{\prime}\right]+\frac{\pi}{\left(1+f_{\tau}^{\prime 2}\left(x_{j}^{p}\right)\right)^{3 / 2}}\left(\partial_{x}^{4}\right)^{3 / 4}\left[\pi_{j}^{p} h\right]\right\|_{2}
$$


with

$$
\begin{aligned}
C_{0,0}\left[\pi_{j}^{p} \bar{\omega}_{1}^{\prime}\right]+\frac{\pi}{\left(1+f_{\tau}^{\prime 2}\left(x_{j}^{p}\right)\right)^{3 / 2}}\left(\partial_{x}^{4}\right)^{3 / 4}\left[\pi_{j}^{p} h\right]= & C_{0,0}\left[\pi_{j}^{p} \bar{\omega}_{1}^{\prime}-\frac{\pi_{j}^{p} h^{\prime \prime \prime}}{\left(1+f_{\tau}^{\prime 2}\left(x_{j}^{p}\right)\right)^{3 / 2}}\right] \\
& -\frac{1}{\left(1+f_{\tau}^{\prime 2}\left(x_{j}^{p}\right)\right)^{3 / 2}} C_{0,0}\left[3\left(\pi_{j}^{p}\right)^{\prime} h^{\prime \prime}+3\left(\pi_{j}^{p}\right)^{\prime \prime} h^{\prime}+\left(\pi_{j}^{p}\right)^{\prime \prime \prime} h\right] \\
& -\frac{1}{2\left(1+f_{\tau}^{\prime 2}\left(x_{j}^{p}\right)\right)^{3 / 2}} \int_{-\pi}^{\pi}\left[\frac{1}{t_{[s]}}-\frac{1}{s / 2}\right]\left(\pi_{j}^{p} h\right)^{\prime \prime \prime}(\cdot-s) \mathrm{d} s .
\end{aligned}
$$

Integration by parts and Lemma A.1 ( $i$ ) lead us to

$$
\left\|C_{0,0}\left[\pi_{j}^{p} \bar{\omega}_{1}^{\prime}\right]+\frac{\pi}{\left(1+f_{\tau}^{\prime 2}\left(x_{j}^{p}\right)\right)^{3 / 2}}\left(\partial_{x}^{4}\right)^{3 / 4}\left[\pi_{j}^{p} h\right]\right\|_{2} \leq C\left\|\pi_{j}^{p} \bar{\omega}_{1}^{\prime}-\frac{\pi_{j}^{p} h^{\prime \prime \prime}}{\left(1+f_{\tau}^{\prime 2}\left(x_{j}^{p}\right)\right)^{3 / 2}}\right\|_{2}+K\|h\|_{H^{2}} .
$$

A straight forward consequence of (4.16) is the following identity

$$
\begin{aligned}
\pi_{j}^{p} \bar{\omega}_{1}^{\prime}-\frac{\pi_{j}^{p} h^{\prime \prime \prime}}{\left(1+f_{\tau}^{\prime 2}\left(x_{j}^{p}\right)\right)^{3 / 2}=} & {\left[\frac{1}{\left(1+f_{\tau}^{\prime 2}\right)^{3 / 2}}-\frac{1}{\left(1+f_{\tau}^{\prime 2}\left(x_{j}^{p}\right)\right)^{3 / 2}}\right] \pi_{j}^{p} h^{\prime \prime \prime}-a_{\mu} \mathbb{A}\left(f_{\tau}\right)\left[\pi_{j}^{p} \bar{\omega}_{1}^{\prime}\right] } \\
& -\frac{3 \pi_{j}^{p} f_{\tau}^{\prime} f_{\tau}^{\prime \prime} h^{\prime \prime}}{\left(1+f_{\tau}^{\prime 2}\right)^{5 / 2}}-a_{\mu} \pi_{j}^{p} T_{\mathrm{lot}}^{A}\left(f_{\tau}\right)\left[\bar{\omega}_{1}\right]+a_{\mu}\left(\mathbb{A}\left(f_{\tau}\right)\left[\pi_{j}^{p} \bar{\omega}_{1}^{\prime}\right]-\pi_{j}^{p} \mathbb{A}\left(f_{\tau}\right)\left[\bar{\omega}_{1}^{\prime}\right]\right) .
\end{aligned}
$$

Using once more the Hölder continuity of $f^{\prime},(3.29)$ and (4.6) (both with $\left.\tau=3 / 4\right)$ together with (4.17) yields that for $p$ sufficiently large

$$
\left\|T_{3}[h]\right\|_{2} \leq C\left\|f_{\tau}^{\prime} C_{0,1}\left(f_{\tau}\right)\left[\pi_{j}^{p} \bar{\omega}_{1}^{\prime}\right]-C_{1,1}\left(f_{\tau}\right)\left[f_{\tau}, \pi_{j}^{p} \bar{\omega}_{1}^{\prime}\right]\right\|_{2}+\frac{\mu}{24}\left\|\pi_{j}^{p} h\right\|_{H^{3}}+K\|h\|_{H^{11 / 12}} .
$$

We are left with the term

$$
f_{\tau}^{\prime} C_{0,1}\left(f_{\tau}\right)\left[\pi_{j}^{p} \bar{\omega}_{1}^{\prime}\right]-C_{1,1}\left(f_{\tau}\right)\left[f_{\tau}, \pi_{j}^{p} \bar{\omega}_{1}^{\prime}\right]=T_{31}[h]-T_{21}[h],
$$

with $T_{21}[h]$ defined above and with

$$
T_{31}[h]:=\left(f_{\tau}^{\prime}-f_{\tau}^{\prime}\left(x_{j}^{p}\right)\right) C_{0,1}\left(f_{\tau}\right)\left[\pi_{j}^{p} \bar{\omega}_{1}^{\prime}\right] .
$$

Since

$$
T_{31}[h]=\chi_{j}^{p}\left(f_{\tau}^{\prime}-f_{\tau}^{\prime}\left(x_{j}^{p}\right)\right) C_{0,1}\left(f_{\tau}\right)\left[\pi_{j}^{p} \bar{\omega}_{1}^{\prime}\right]-\left(f_{\tau}^{\prime}-f_{\tau}^{\prime}\left(x_{j}^{p}\right)\right) \int_{-\pi}^{\pi} \frac{\delta_{[\cdot, s]} \chi_{j}^{p} / s}{1+\left(\delta_{[\cdot, s]} f_{\tau} / s\right)^{2}}\left(\pi_{j}^{p} \bar{\omega}_{1}^{\prime}\right)(\cdot-s) \mathrm{d} s,
$$

the estimate (4.18) and Lemma A.1 (i) for the first term, respectively integration by parts for the second term lead us, for $p$ sufficiently large, to

$$
C\left\|T_{31}[h]\right\|_{2} \leq \frac{\mu}{24}\left\|\pi_{j}^{p} h\right\|_{H^{3}}+K\|h\|_{H^{11 / 12}} .
$$

Gathering (4.20) (which is valid also for $C\left\|T_{21}[h]\right\|_{2}$ provided that we choose a larger $p$ if required), (4.22), and (4.23), we conclude that

$$
\left\|T_{3}[h]\right\|_{2} \leq \frac{\mu}{6}\left\|\pi_{j}^{p} h\right\|_{H^{3}}+K\|h\|_{H^{11 / 12}}
$$

provided that $p$ is sufficiently large. The estimate (4.13) follows now from (4.19), (4.21), and (4.24).

Step 2: The second leading order term. Given $1 \leq j \leq 2^{p+1}$ and $\tau \in[0,1]$, let

$$
\mathbb{A}_{j, \tau}^{2}:=-\frac{1}{\left(1+f_{\tau}^{\prime 2}\left(x_{j}^{p}\right)\right)^{5 / 2}}\left(\partial_{x}^{4}\right)^{3 / 4}
$$

where $x_{j}^{p} \in I_{j}^{p}$. Similarly as in the previous step, it follows that

$$
\left\|\pi_{j}^{p} C_{0,1}\left(f_{\tau}\right)\left[\bar{\omega}_{1}^{\prime}\right]-\left.\pi \mathbb{A}_{j, \tau}^{2}\left[\pi_{j}^{p} h\right]\right|_{2} \leq \frac{\mu}{2}\right\| \pi_{j}^{p} h\left\|_{H^{3}}+K\right\| h \|_{H^{11 / 4}}
$$

for all $j \in\left\{1, \ldots, 2^{p+1}\right\}, \tau \in[0,1]$, and $h \in H^{3}(\mathbb{S})$, provided that $p$ is sufficiently large. 
The desired claim (4.9) follows from (4.11), (4.12), (4.13), and (4.25).

We are now in a position to prove (4.2).

Theorem 4.3. Given $f \in H^{2}(\mathbb{S})$, it holds that

$$
-\Phi_{\sigma}(f) \in \mathcal{H}\left(H^{3}(\mathbb{S}), L_{2}(\mathbb{S})\right) .
$$

Proof. Let $\Phi_{\sigma}^{c}(f)=\Phi_{\sigma, 1}^{c}(f)+\Phi_{\sigma, 2}^{c}(f)$ denote the complexification of $\Phi_{\sigma}(f)$ (the Sobolev spaces where $\Phi_{\sigma}^{c}(f)$ acts are now complex valued). In view of [46, Corollary 2.1.3] is suffices to show that $-\Phi_{\sigma}^{c}(f) \in$ $\mathcal{H}\left(H^{3}(\mathbb{S}), L_{2}(\mathbb{S})\right)$. Moreover, for the choice $\tau=3 / 4$ in Proposition $4.1(b)$, we obtain together with Lemma 3.1, that $\Phi_{\sigma, 2}^{c}(f) \in \mathcal{L}\left(H^{11 / 4}(\mathbb{S}), L_{2}(\mathbb{S})\right)$. Since $\left[L_{2}(\mathbb{S}), H^{3}(\mathbb{S})\right]_{11 / 12}=H^{11 / 4}(\mathbb{S})$, cf. (3.30), by $[5$, Theorem I.1.3.1 (ii)] we only need to show that

$$
-\Phi_{\sigma, 1}^{c}(f) \in \mathcal{H}\left(H^{3}(\mathbb{S}), L_{2}(\mathbb{S})\right) .
$$

Recalling [5, Remark I.1.21 (a) ], we are left to find constants $\omega>0$ and $\kappa \geq 1$ such that

$$
\begin{aligned}
& \omega-\Phi_{\sigma, 1}^{c}(f) \in \operatorname{Isom}\left(H^{3}(\mathbb{S}), L_{2}(\mathbb{S})\right), \\
& \kappa\left\|\left(\lambda-\Phi_{\sigma, 1}^{c}(f)\right)[h]\right\|_{2} \geq|\lambda| \cdot\|h\|_{2}+\|h\|_{H^{3}} \quad \forall h \in H^{3}(\mathbb{S}) \text { and } \operatorname{Re} \lambda \geq \omega .
\end{aligned}
$$

Let $a>1$ be chosen such that

$$
\frac{1}{a} \leq \frac{1}{\left(1+\left\|f^{\prime}\right\|_{\infty}^{2}\right)^{3 / 2}} \leq a
$$

For each $\alpha \in\left[a^{-1}, a\right]$, let $\mathbb{A}_{\alpha}: H^{3}(\mathbb{S}) \rightarrow L_{2}(\mathbb{S})$ denote operator $\mathbb{A}_{\alpha}:=-\alpha\left(\partial_{x}^{4}\right)^{3 / 4}$. Then it is easy to see that for $\kappa^{\prime}:=1+a$ the following hold

$$
\begin{aligned}
& \lambda-\mathbb{A}_{\alpha} \in \operatorname{Isom}\left(H^{3}(\mathbb{S}), L_{2}(\mathbb{S})\right) \quad \forall \operatorname{Re} \lambda \geq 1, \\
& \kappa^{\prime}\left\|\left(\lambda-\mathbb{A}_{\alpha}\right)[h]\right\|_{2} \geq|\lambda| \cdot\|h\|_{2}+\|h\|_{H^{3}} \quad \forall h \in H^{3}(\mathbb{S}) \text { and } \operatorname{Re} \lambda \geq 1 .
\end{aligned}
$$

Taking $\mu:=1 /\left(2 \kappa^{\prime}\right)$ in Theorem 4.2 , we find $p \geq 3$, a $p$-partition of unity $\left\{\pi_{j}^{p}: 1 \leq j \leq 2^{p+1}\right\}$, a constant $K=K(p)$, and for each $j \in\left\{1, \ldots, 2^{p+1}\right\}$ and $\tau \in[0,1]$ operators $\mathbb{A}_{j, \tau}^{c} \in \mathcal{L}\left(H^{3}(\mathbb{S}), L_{2}(\mathbb{S})\right)\left(\mathbb{A}_{j, \tau}^{c}\right.$ is the complexification of $\mathbb{A}_{j, \tau}$ defined in (4.10)) such that

$$
\left\|\pi_{j}^{p} \Phi_{\sigma, 1}^{c}(\tau f)[h]-\mathbb{A}_{j, \tau}^{c}\left[\pi_{j}^{p} h\right]\right\|_{2} \leq \mu\left\|\pi_{j}^{p} h\right\|_{H^{3}}+K\|h\|_{H^{11 / 4}}
$$

for all $j \in\left\{1, \ldots, 2^{p+1}\right\}, \tau \in[0,1]$, and $h \in H^{3}(\mathbb{S})$. We note that the relations (4.29) and (4.30) are both valid for $\mathbb{A}_{j, \tau}^{c}$ as $\mathbb{A}_{j, \tau}^{c} \in\left\{\mathbb{A}_{\alpha}: \alpha \in\left[a^{-1}, a\right]\right\}$. It now follows from (4.30) and (4.31) that

$$
\begin{aligned}
\kappa^{\prime}\left\|\pi_{j}^{p}\left(\lambda-\Phi_{\sigma, 1}^{c}(\tau f)\right)[h]\right\|_{2} & \geq \kappa^{\prime}\left\|\left(\lambda-\mathbb{A}_{j, \tau}^{c}(f)\right)\left[\pi_{j}^{p} h\right]\right\|_{2}-\kappa^{\prime}\left\|\pi_{j}^{p} \Phi_{\sigma, 1}^{c}(\tau f)[h]-\mathbb{A}_{j, \tau}^{c}\left[\pi_{j}^{p} h\right]\right\|_{2} \\
& \geq|\lambda| \cdot\left\|\pi_{j}^{p} h\right\|_{2}+\frac{1}{2}\left\|\pi_{j}^{p} h\right\|_{H^{3}}-\kappa^{\prime} K\|h\|_{H^{11 / 4}}
\end{aligned}
$$

for all $j \in\left\{1, \ldots, 2^{p+1}\right\}, \tau \in[0,1]$, and $h \in H^{3}(\mathbb{S})$. Since for each $k \in \mathbb{N}$

$$
\left[h \mapsto \max _{1 \leq j \leq 2^{p+1}}\left\|\pi_{j}^{p} h\right\|_{H^{k}}\right]: H^{k}(\mathbb{S}) \rightarrow \mathbb{R},
$$

defines a norm equivalent to the standard $H^{k}(\mathbb{S})$-norm, cf. [47, Remark 4.1], Young's inequality together with (3.30) enables us to conclude from the previous inequality the existence of constants $\omega>1$ and $\kappa \geq 1$ with

$$
\kappa\left\|\left(\lambda-\Phi_{\sigma, 1}^{c}(\tau f)\right)[h]\right\|_{2} \geq|\lambda| \cdot\|h\|_{2}+\|h\|_{H^{3}} \quad \forall h \in H^{3}(\mathbb{S}), \tau \in[0,1], \text { and } \operatorname{Re} \lambda \geq \omega .
$$

Choosing $\tau=1$ in (4.32) we obtain (4.28). Moreover, the estimate (4.32) for $\lambda=\omega,(4.29)\left(\Phi_{\sigma, 1}^{c}(\tau f)=\mathbb{A}_{1}\right.$ for $\tau=0$ ), and the method of continuity [5, Proposition I.1.1.1] ensure that the property (4.27) also holds and the proof is complete. 
We now come to the proof our first main result which uses on the one hand the abstract theory for quasilinear parabolic problems outlined in [1-5] (see also [50, Theorem 1.1]), and on the other hand a parameter trick which has been employed in various versions in $[8,34,47-49,57]$ in the context of improving the regularity of solutions to certain parabolic evolution equations. We point out that the parameter trick can only be used because the uniqueness claim of Theorem 1.1 holds in the setting of classical solution (the solutions in Theorem 1.1 possess though additional Hölder regularity properties, see the proof of Theorem 1.1).

Proof of Theorem 1.1. Let $\mathbb{E}_{1}:=H^{3}(\mathbb{S}), \mathbb{E}_{0}:=L_{2}(\mathbb{S}), \beta:=2 / 3$ and $\alpha:=r / 3$. Then $\mathbb{E}_{1} \hookrightarrow \mathbb{E}_{0}$ is a compact embedding, $0<\beta<\alpha<1$, and it follows from Theorem 4.3 and (4.8) that the abstract result [50, Theorem 1.1] may be applied in the context of the Muskat problem (4.1). Hence, given $f_{0} \in H^{r}(\mathbb{S})=$ $\left[L_{2}(\mathbb{S}), H^{3}(\mathbb{S})\right]_{\alpha},(4.1)$ possesses a unique classical solution $f=f\left(\cdot ; f_{0}\right)$, that is

$$
f \in C\left(\left[0, T_{+}\left(f_{0}\right)\right), H^{r}(\mathbb{S})\right) \cap C\left(\left(0, T_{+}\left(f_{0}\right)\right), H^{3}(\mathbb{S})\right) \cap C^{1}\left(\left(0, T_{+}\left(f_{0}\right)\right), L_{2}(\mathbb{S})\right),
$$

where $T_{+}\left(f_{0}\right) \leq \infty$, which has the property that

$$
f \in C^{\alpha-\beta}\left([0, T], H^{2}(\mathbb{S})\right) \quad \text { for all } T<T_{+}\left(f_{0}\right) .
$$

Concerning the uniqueness statement of Theorem $1.1(i)$, it suffices to prove that if $T>0$ and

$$
f \in C\left([0, T], H^{r}(\mathbb{S})\right) \cap C\left((0, T], H^{3}(\mathbb{S})\right) \cap C^{1}\left((0, T], L_{2}(\mathbb{S})\right)
$$

solves (4.1) pointwise, then

$$
f \in C^{\eta}\left([0, T], H^{2}(\mathbb{S})\right) \quad \text { for } \eta:=\frac{r-2}{r+1},
$$

cf. [50, Theorem 1.1]. Let thus $f$ be a solution to (4.1) which satisfies (4.33). Since $f \in C\left([0, T], H^{r}(\mathbb{S})\right)$ and $r>2$, we deduce from the Theorems 3.3 and 3.5 via interpolation that

$$
\sup _{t \in[0, T]}\left\|\left(1+a_{\mu} \mathbb{A}(f)\right)^{-1}\right\|_{\mathcal{L}\left(\widehat{H}^{r-2}(\mathbb{S})\right)} \leq C .
$$

Since $\langle\kappa(f)\rangle=0$ and $\sup _{t \in[0, T]}\|\kappa(f)\|_{H^{r-2}} \leq C$, it follows for $\bar{\omega}_{1}:=\bar{\omega}_{1}(f)[f]=\left(1+a_{\mu} \mathbb{A}(f)\right)^{-1}[\kappa(f)]$ (see Proposition 4.1) that

$$
\sup _{t \in[0, T]}\left\|\bar{\omega}_{1}\right\|_{H^{r-2}} \leq C
$$

We next show that

$$
\sup _{t \in(0, T]}\left\|\Phi_{\sigma, 1}(f)[f]\right\|_{H^{-1}}+\sup _{t \in(0, T]}\left\|\Phi_{\sigma, 2}(f)[f]\right\|_{H^{-1}} \leq C .
$$

It follows from the definitions of $\Phi_{\sigma, 1}$ and $\bar{\omega}_{1}$ that

$$
\Phi_{\sigma, 1}(f)[f]=f^{\prime} \mathbb{B}_{1}(f)\left[\bar{\omega}_{1}^{\prime}\right]-\mathbb{B}_{2}(f)\left[\bar{\omega}_{1}^{\prime}\right]+\frac{1}{\pi}\left(C_{0,1}(f)\left[\bar{\omega}_{1}^{\prime}\right]+f^{\prime} C_{1,1}(f)\left[f, \bar{\omega}_{1}^{\prime}\right]\right), \quad t \in(0, T] .
$$

Using integration by parts, it is not difficult to derive, with the help of (4.35), the estimate

$$
\sup _{t \in(0, T]}\left\|f^{\prime} \mathbb{B}_{1}(f)\left[\bar{\omega}_{1}^{\prime}\right]\right\|_{2}+\sup _{t \in(0, T]}\left\|\mathbb{B}_{2}(f)\left[\bar{\omega}_{1}^{\prime}\right]\right\|_{2} \leq C,
$$

and we are left to consider the terms $C_{0,1}(f)\left[\bar{\omega}_{1}^{\prime}\right]$ and $f^{\prime} C_{1,1}(f)\left[f, \bar{\omega}_{1}^{\prime}\right]$. Since $\bar{\omega}_{1} \in H^{1}(\mathbb{S})$ for $t \in(0, T]$, it is shown in Lemma 3.1 that $C_{1,1}(f)\left[f, \bar{\omega}_{1}\right] \in H^{1}(\mathbb{S})$ with

$$
f^{\prime} C_{1,1}(f)\left[f, \bar{\omega}_{1}^{\prime}\right]=f^{\prime}\left(C_{1,1}(f)\left[f, \bar{\omega}_{1}\right]\right)^{\prime}-f^{\prime} C_{1,1}(f)\left[f^{\prime}, \bar{\omega}_{1}\right]+2 f^{\prime} C_{3,2}(f, f)\left[f^{\prime}, f, f, \bar{\omega}_{1}\right] .
$$

We estimate the terms on the right hand side of the latter identity in the $H^{-1}$-norm one by one. Given $\varphi \in H^{1}(\mathbb{S})$, integration by parts, (4.35), and Lemma A.1 ( $i$ ) yield

$$
\left|\int_{-\pi}^{\pi} f^{\prime}\left(C_{1,1}(f)\left[f, \bar{\omega}_{1}\right]\right)^{\prime} \varphi \mathrm{d} x\right| \leq\left|\int_{-\pi}^{\pi} f^{\prime \prime} C_{1,1}(f)\left[f, \bar{\omega}_{1}\right] \varphi \mathrm{d} x\right|+\left|\int_{-\pi}^{\pi} f^{\prime} C_{1,1}(f)\left[f, \bar{\omega}_{1}\right] \varphi^{\prime} \mathrm{d} x\right| \leq C\|\varphi\|_{H^{1}},
$$


and therewith

$$
\sup _{t \in(0, T]}\left\|f^{\prime}\left(C_{1,1}(f)\left[f, \bar{\omega}_{1}\right]\right)^{\prime}\right\|_{H^{-1}} \leq C .
$$

In order to estimate $f^{\prime} C_{1,1}(f)\left[f^{\prime}, \bar{\omega}_{1}\right]$ we write

$$
C_{1,1}(f)\left[f^{\prime}, \bar{\omega}_{1}\right]=T_{1}-T_{2}-T_{3},
$$

where

$$
\begin{aligned}
& T_{1}:=\int_{0}^{\pi} \frac{\delta_{[x, s]} f^{\prime} / s}{1+\left(\delta_{[x, s]} f / s\right)^{2}} \frac{\bar{\omega}_{1}(x-s)-\bar{\omega}_{1}(x+s)}{s} \mathrm{~d} s \\
& T_{2}:=\int_{0}^{\pi} \frac{1}{1+\left(\delta_{[x, s]} f / s\right)^{2}} \frac{f^{\prime}(x+s)-2 f^{\prime}(x)+f^{\prime}(x-s)}{s} \frac{\bar{\omega}_{1}(x+s)}{s} \mathrm{~d} s \\
& T_{3}:=\int_{0}^{\pi} \frac{\left[\left(\delta_{[x, s]} f / s\right)-\left(\delta_{[x,-s]} f / s\right)\right]\left(\delta_{[x,-s]} f^{\prime} / s\right)}{\left[1+\left(\delta_{[x, s]} f / s\right)^{2}\right]\left[1+\left(\delta_{[x,-s]} f / s\right)^{2}\right]} \frac{f(x+s)-2 f(x)+f(x-s)}{s} \frac{\bar{\omega}_{1}(x+s)}{s} \mathrm{~d} s .
\end{aligned}
$$

Given $\varphi \in H^{1}(\mathbb{S})$, Fubini's theorem yields for $t \in(0, T]$

$$
\begin{aligned}
\left|\int_{-\pi}^{\pi} f^{\prime} T_{1} \varphi \mathrm{d} x\right| & \leq C\|\varphi\|_{H^{1}} \int_{0}^{\pi} \int_{-\pi}^{\pi}\left|\frac{\delta_{[x, s]} f^{\prime}}{s}\right| \cdot\left|\frac{\bar{\omega}_{1}(x-s)-\bar{\omega}_{1}(x+s)}{s}\right| \mathrm{d} x \mathrm{~d} s \\
& \leq C\|\varphi\|_{H^{1}} \int_{0}^{\pi} \frac{1}{s^{2}}\left(\int_{-\pi}^{\pi}\left|f^{\prime}-\tau_{s} f^{\prime}\right|^{2} \mathrm{~d} x\right)^{1 / 2}\left(\int_{-\pi}^{\pi}\left|\tau_{s} \bar{\omega}_{1}-\tau_{-s} \bar{\omega}_{1}\right|^{2} \mathrm{~d} x\right)^{1 / 2} \mathrm{~d} s \\
& \leq C\|\varphi\|_{H^{1}} \int_{0}^{\pi} \frac{1}{s^{2}}\left(\sum_{k \in \mathbb{Z}}|k|^{2}|\widehat{f}(k)|^{2}\left|e^{i k s}-1\right|^{2}\right)^{1 / 2}\left(\sum_{k \in \mathbb{Z}}\left|\widehat{\bar{\omega}}_{1}(k)\right|^{2}\left|e^{i 2 k s}-1\right|^{2}\right)^{1 / 2} \mathrm{~d} s,
\end{aligned}
$$

and since $\left|e^{i \xi}-1\right| \leq C|\xi|$, respectively $\left|e^{i \xi}-1\right| \leq C|\xi|^{r-2}$, for all $\xi \in \mathbb{R}$, the latter inequality together with (4.35) leads to

$$
\left\|f^{\prime} T_{1}\right\|_{H^{-1}} \leq C\|f\|_{H^{2}}\left\|\bar{\omega}_{1}\right\|_{H^{r-2}} \int_{0}^{\pi} s^{r-3} \mathrm{~d} s \leq C .
$$

Arguing along the same lines we find for $t \in(0, T]$, in view of $\left|e^{i \xi}-2+e^{-i \xi}\right| \leq C|\xi|^{r-1}$ for all $\xi \in \mathbb{R}$, that

$$
\begin{aligned}
\left|\int_{-\pi}^{\pi} f^{\prime} T_{2} \varphi \mathrm{d} x\right| & \leq C\|\varphi\|_{H^{1}}\left\|\bar{\omega}_{1}\right\|_{2} \int_{0}^{\pi} \frac{1}{s^{2}}\left(\int_{-\pi}^{\pi}\left|\tau_{-s} f^{\prime}-2 f^{\prime}+\tau_{s} f^{\prime}\right|^{2} \mathrm{~d} x\right)^{1 / 2} \mathrm{~d} s \\
& \leq C\|\varphi\|_{H^{1}} \int_{0}^{\pi} \frac{1}{s^{2}}\left(\sum_{k \in \mathbb{Z}}|k|^{2}|\widehat{f}(k)|^{2}\left|e^{i k s}-2+e^{-i k s}\right|^{2}\right)^{1 / 2} \mathrm{~d} s \\
& \leq C\|\varphi\|_{H^{1}}\|f\|_{H^{r}} \int_{0}^{\pi} s^{r-3} \mathrm{~d} s
\end{aligned}
$$

and therewith

$$
\left\|f^{\prime} T_{2}\right\|_{H^{-1}} \leq C
$$

Finally, the inequality $\left|e^{i \xi}-2+e^{-i \xi}\right| \leq C|\xi|^{2}$ for all $\xi \in \mathbb{R}$ together with the Sobolev embedding $H^{r-1}(\mathbb{S}) \hookrightarrow \mathrm{C}^{r-3 / 2}(\mathbb{S})$ for $r \neq 5 / 2$, yield for $t \in(0, T]$ that

$$
\begin{aligned}
\left|\int_{-\pi}^{\pi} f^{\prime} T_{3} \varphi \mathrm{d} x\right| & \leq C\|\varphi\|_{H^{1}}\left\|\bar{\omega}_{1}\right\|_{2}\|f\|_{H^{r}} \int_{0}^{\pi} s^{\min \{-2, r-9 / 2\}}\left(\int_{-\pi}^{\pi}\left|\tau_{-s} f-2 f+\tau_{s} f\right|^{2} \mathrm{~d} x\right)^{1 / 2} \mathrm{~d} s \\
& \leq C\|\varphi\|_{H^{1}} \int_{0}^{\pi} s^{\min \{-2, r-9 / 2\}}\left(\sum_{k \in \mathbb{Z}}|\widehat{f}(k)|^{2}\left|e^{i k s}-2+e^{-i k s}\right|^{2}\right)^{1 / 2} \mathrm{~d} s
\end{aligned}
$$




$$
\leq C\|\varphi\|_{H^{1}}\|f\|_{H^{2}} \int_{0}^{\pi} s^{\min \{0, r-5 / 2\}} \mathrm{d} s
$$

hence

$$
\left\|f^{\prime} T_{3}\right\|_{H^{-1}} \leq C .
$$

The latter estimate clearly holds also for $r=5 / 2$. We have thus shown that

$$
\sup _{t \in(0, T]}\left\|f^{\prime} C_{1,1}(f)\left[f^{\prime}, \bar{\omega}_{1}\right]\right\|_{H^{-1}} \leq C
$$

holds true. Similarly

$$
\sup _{t \in(0, T]}\left\|f^{\prime} C_{3,2}(f, f)\left[f^{\prime}, f, f, \bar{\omega}_{1}\right]\right\|_{H^{-1}} \leq C .
$$

Gathering (4.38)-(4.40), it follows that

$$
\sup _{t \in(0, T]}\left\|f^{\prime} C_{1,1}(f)\left[f, \bar{\omega}_{1}^{\prime}\right]\right\|_{H^{-1}} \leq C .
$$

Similarly, we get

$$
\sup _{t \in(0, T]}\left\|C_{0,1}(f)\left[\bar{\omega}_{1}^{\prime}\right]\right\|_{H^{-1}} \leq C
$$

and (4.37), (4.41), and (4.42) lead to

$$
\sup _{t \in(0, T]}\left\|\Phi_{\sigma, 1}(f)[f]\right\|_{H^{-1}} \leq C .
$$

We now consider the second term $\Phi_{\sigma, 2}$. Given $t \in(0, T]$, it holds

$$
\Phi_{\sigma, 2}(f)[f]=\mathbb{B}(f)\left[\bar{\omega}_{2}(f)[f]\right]=-\Theta \mathbb{B}(f)\left[\left(1+a_{\mu} \mathbb{A}(f)\right)^{-1}\left[f^{\prime}\right]\right]+a_{\mu} \mathbb{B}(f)\left[\left(1+a_{\mu} \mathbb{A}(f)\right)^{-1}\left[T_{\text {lot }}^{A}(f)\left[\bar{\omega}_{1}\right]\right]\right],
$$

and Lemma 3.1 together with Theorem 3.3 yields

$$
\left\|\mathbb{B}(f)\left[\left(1+a_{\mu} \mathbb{A}(f)\right)^{-1}\left[f^{\prime}\right]\right]\right\|_{2} \leq C\left\|\left(1+a_{\mu} \mathbb{A}(f)\right)^{-1}\left[f^{\prime}\right]\right\|_{2} \leq C\left\|f^{\prime}\right\|_{2} \leq C \quad \forall t \in[0, T] .
$$

We now estimate $\left\|\mathbb{B}(f)\left[\bar{\omega}_{3}\right]\right\|_{H^{-1}}$, where $\bar{\omega}_{3}:=\bar{\omega}_{3}(f):=\left(1+a_{\mu} \mathbb{A}(f)\right)^{-1}\left[T_{\text {lot }}^{A}(f)\left[\bar{\omega}_{1}\right]\right] \in \widehat{L}_{2}(\mathbb{S})$ for $t \in(0, T]$. We begin by showing that the function $T_{\text {lot }}^{A}(f)\left[\bar{\omega}_{1}\right] \in \widehat{L}_{2}(\mathbb{S})$, see (3.28), satisfies

$$
\sup _{t \in(0, T]}\left\|T_{\mathrm{lot}}^{A}(f)\left[\bar{\omega}_{1}\right]\right\|_{1} \leq C .
$$

Firstly we consider the difference $\left(f^{\prime} \mathbb{B}_{2}(f)\left[\bar{\omega}_{1}\right]\right)^{\prime}-f^{\prime} \mathbb{B}_{2}(f)\left[\bar{\omega}_{1}^{\prime}\right]$, which we estimate, in view of $(4.35)$ and Lemma 3.2, as follows

$$
\begin{aligned}
\left\|\left(f^{\prime} \mathbb{B}_{2}(f)\left[\bar{\omega}_{1}\right]\right)^{\prime}-f^{\prime} \mathbb{B}_{2}(f)\left[\bar{\omega}_{1}^{\prime}\right]\right\|_{1} & \leq\left\|f^{\prime \prime} \mathbb{B}_{2}(f)\left[\bar{\omega}_{1}\right]\right\|_{1}+\left\|f^{\prime}\right\|_{\infty}\left\|\left(\mathbb{B}_{2}(f)\left[\bar{\omega}_{1}\right]\right)^{\prime}-\mathbb{B}_{2}(f)\left[\bar{\omega}_{1}^{\prime}\right]\right\|_{1} \\
& \leq\left\|f^{\prime \prime}\right\|_{2}\left\|\mathbb{B}_{2}(f)\left[\bar{\omega}_{1}\right]\right\|_{2}+C\left\|\left(\mathbb{B}_{2}(f)\left[\bar{\omega}_{1}\right]\right)^{\prime}-\mathbb{B}_{2}(f)\left[\bar{\omega}_{1}^{\prime}\right]\right\|_{1} \\
& \leq C\left(1+\left\|\left(\mathbb{B}_{2}(f)\left[\bar{\omega}_{1}\right]\right)^{\prime}-\mathbb{B}_{2}(f)\left[\bar{\omega}_{1}^{\prime}\right]\right\|_{1}\right) .
\end{aligned}
$$

Secondly, it is not difficult to see that

$$
\left\|\left(\mathbb{B}_{1}(f)\left[\bar{\omega}_{1}\right]\right)^{\prime}-\mathbb{B}_{1}(f)\left[\bar{\omega}_{1}^{\prime}\right]\right\|_{1}+\left\|\left(\mathbb{B}_{2}(f)\left[\bar{\omega}_{1}\right]\right)^{\prime}-\mathbb{B}_{2}(f)\left[\bar{\omega}_{1}^{\prime}\right]\right\|_{1} \leq C\left\|\bar{\omega}_{1}\right\|_{2} \leq C .
$$

We still need to estimate the terms of $T_{\text {lot }}^{A}(f)\left[\bar{\omega}_{1}\right]$ defined by means of the operators $C_{n, m}$ introduced in Lemma A.1. This is done as follows

$$
\begin{aligned}
& \left\|f^{\prime \prime} C_{0,1}(f)\left[\bar{\omega}_{1}\right]\right\|_{1} \leq\left\|f^{\prime \prime}\right\|_{2}\left\|C_{0,1}(f)\left[\bar{\omega}_{1}\right]\right\|_{2} \leq C\left\|\bar{\omega}_{1}\right\|_{2} \leq C, \\
& \left\|f^{\prime} C_{2,2}(f, f)\left[f^{\prime}, f, \bar{\omega}_{1}\right]\right\|_{1}+\left\|C_{1,1}(f)\left[f^{\prime}, \bar{\omega}_{1}\right]\right\|_{1}+\left\|f^{\prime} C_{3,2}(f, f)\left[f^{\prime}, f, f, \bar{\omega}_{1}\right]\right\|_{1} \leq C,
\end{aligned}
$$

the last estimate following in a similar way as (4.39). Altogether, (4.45) holds true.

Given $t \in(0, T]$, we compute for $\varphi \in H^{1}(\mathbb{S})$ that

$$
\left|\int_{-\pi}^{\pi} \bar{\omega}_{3} \varphi \mathrm{d} x\right|=\left|\int_{-\pi}^{\pi}\left(1+a_{\mu} \mathbb{A}(f)\right)^{-1}\left[T_{\mathrm{lot}}^{A}(f)\left[\bar{\omega}_{1}\right]\right] P \varphi \mathrm{d} x\right|
$$




$$
\begin{aligned}
& =\left|\int_{-\pi}^{\pi} T_{\mathrm{lot}}^{A}(f)\left[\bar{\omega}_{1}\right]\left(1+a_{\mu}(\widehat{\mathbb{A}(f)})^{*}\right)^{-1}[P \varphi] \mathrm{d} x\right| \\
& \leq\left\|T_{\mathrm{lot}}^{A}(f)\left[\bar{\omega}_{1}\right]\right\|_{1}\left\|\left(1+a_{\mu}(\widehat{\mathbb{A}(f)})^{*}\right)^{-1}\right\|_{\mathcal{L}\left(\widehat{H}^{1}(\mathbb{S})\right)}\|P \varphi\|_{H^{1}},
\end{aligned}
$$

where $P$ is the orthogonal projection on $\widehat{L}_{2}(\mathbb{S})$. This inequality together with Theorem 3.6 and $(4.45)$ implies

$$
\sup _{t \in(0, T]}\left\|\bar{\omega}_{3}\right\|_{H^{-1}} \leq C
$$

Since for $t \in(0, T]$ and $\varphi \in H^{1}(\mathbb{S})$

$$
\left|\int_{-\pi}^{\pi} \mathbb{B}(f)\left[\bar{\omega}_{3}\right] \varphi \mathrm{d} x\right|=\left|\int_{-\pi}^{\pi} \bar{\omega}_{3}(\mathbb{B}(f))^{*}[\varphi] \mathrm{d} x\right| \leq\left\|\bar{\omega}_{3}\right\|_{H^{-1}}\left\|(\mathbb{B}(f))^{*}\right\|_{\mathcal{L}\left(\widehat{H}^{1}(\mathbb{S})\right)}\|\varphi\|_{H^{1}},
$$

Lemma 3.7 together with (4.46) lead us to

$$
\sup _{t \in(0, T]}\left\|\mathbb{B}(f)\left[\bar{\omega}_{3}\right]\right\|_{H^{-1}} \leq C .
$$

In view of (4.44) and (4.47) we conclude that

$$
\sup _{t \in(0, T]}\left\|\Phi_{\sigma, 2}(f)[f]\right\|_{H^{-1}} \leq C,
$$

and the claim (4.36) follows from (4.43) and (4.48).

Recalling that $f \in \mathrm{C}^{1}\left((0, T], L_{2}(\mathbb{S})\right) \cap \mathrm{C}\left([0, T], H^{r}(\mathbb{S})\right)$, (4.36) yields $f \in \mathrm{BC}^{1}\left((0, T], H^{-1}(\mathbb{S})\right)$ and the property (4.34) is now a straight forward consequence of (3.30). This proves the uniqueness claim in Theorem 1.1 and herewith the assertion $(i)$. The claim (ii) follows directly from [50, Theorem 1.1], while the parabolic smoothing property stated at (iii) is obtain by using a parameter trick in the same way as in the proof of [49, Theorem 1.3]. The proof of Theorem 1.1 is now complete.

\section{The Muskat Problem Without Surface Tension Effects}

We now investigate the evolution problem (1.1) in the absence of the surface tension effects, that is for $\sigma=0$. One of the main features of the Muskat problem with surface tension, namely the quasilinear character, seems to be lost as the curvature term disappears from the equations. Nevertheless, we show below that (1.1) can be recast as a fully nonlinear and nonlocal evolution problem

$$
\dot{f}(t)=\Phi(f(t)), \quad t \geq 0, \quad f(0)=f_{0},
$$

with $[f \mapsto \Phi(f)] \in C^{\omega}\left(H^{2}(\mathbb{S}), H^{1}(\mathbb{S})\right)$ defined in (5.5). While the Muskat problem with surface tension is parabolic regardless of the initial data that are considered, in the case when $\sigma=0$ we can prove that the Fréchet derivative $\partial \Phi\left(f_{0}\right)$ generates a strongly continuous and analytic semigroup in $\mathcal{L}\left(H^{1}(\mathbb{S})\right)$, more precisely that

$$
-\partial \Phi\left(f_{0}\right) \in \mathcal{H}\left(H^{2}(\mathbb{S}), H^{1}(\mathbb{S})\right)
$$

only when requiring that the initial data $f_{0} \in H^{2}(\mathbb{S})$ are chosen such that the Rayleigh-Taylor condition is satisfied. Establishing (5.2) is the first goal of this section and this necessitates some preparations.

To begin, we solve the Eq. $(1.1 \mathrm{a})_{2}$, which is, up to a factor of 2, equivalent to

$$
\left(1+a_{\mu} \mathbb{A}(f)\right)[\bar{\omega}]=-c_{\Theta} f^{\prime},
$$

where

$$
c_{\Theta}:=\frac{k \Theta}{\mu_{-}+\mu_{+}} .
$$


It is worth mentioning that in order to solve (5.3) for $\bar{\omega}$ in $\widehat{H}^{1}(\mathbb{S})$ it is required in Theorem 3.5 that the left hand side belongs to $\widehat{H}^{1}(\mathbb{S})$, that is $f \in H^{2}(\mathbb{S})$, and this is precisely the regularity required also for the function in the argument of $\mathbb{A}$. Hence, (5.3) is no longer quasilinear, unless $a_{\mu}=0$, see [47].

Proposition 5.1. Given $f \in H^{2}(\mathbb{S})$, there exists a unique solution $\bar{\omega}:=\bar{\omega}(f) \in \widehat{H}^{1}(\mathbb{S})$ to $(5.3)$ and

$$
\bar{\omega} \in C^{\omega}\left(H^{2}(\mathbb{S}), \widehat{H}^{1}(\mathbb{S})\right) .
$$

Proof. Theorem 3.5 implies that

$$
\bar{\omega}(f):=-c_{\Theta}\left(1+a_{\mu} \mathbb{A}(f)\right)^{-1}\left[f^{\prime}\right]
$$

is the unique solution to $(5.3)$ in $\widehat{H}^{1}(\mathbb{S})$, and the regularity property (5.4) follows from Lemma 3.2.

In view of Proposition 5.1, (1.1) is equivalent to the Eq. (5.1), where $\Phi: H^{2}(\mathbb{S}) \rightarrow \widehat{H}^{1}(\mathbb{S})$ is given by

$$
\Phi(f):=\mathbb{B}(f)[\bar{\omega}(f)]=-c_{\Theta} \mathbb{B}(f)\left[\left(1+a_{\mu} \mathbb{A}(f)\right)^{-1}\left[f^{\prime}\right]\right],
$$

and it satisfies

$$
\Phi \in C^{\omega}\left(H^{2}(\mathbb{S}), \widehat{H}^{1}(\mathbb{S})\right) \cap C^{\omega}\left(H^{2}(\mathbb{S}), H^{1}(\mathbb{S})\right),
$$

cf. (3.6) and (5.4). With respect to our goal of proving Theorem 1.5, the fact that $\Phi$ maps in $\widehat{H}^{1}(\mathbb{S})$ is not relevant, and therefore we shall not rely in this part on this property, but consider instead $\Phi$ as a mapping in $H^{1}(\mathbb{S})$. In view of Lemma 2.2 and Proposition 5.1 the Rayleigh-Taylor condition (1.3) can be reformulated as

$$
a_{\mathrm{RT}}:=c_{\Theta}+a_{\mu} \Phi\left(f_{0}\right)>0 .
$$

Indeed, recalling $(2.1 \mathrm{a})_{2}$ and $(2.2)_{2}$, we obtain that

$$
\nabla p_{ \pm}(t, x, f(t, x)+t V)=-\frac{\mu_{ \pm}}{k}\left(V_{ \pm}(t, x, f(t, x))+(0, V)\right)-\left(0, \rho_{ \pm} g\right), \quad x \in \mathbb{R},
$$

with $V_{ \pm}$as defined in (2.5). The relation (5.7) follows now from (1.3), (5.3), and (5.5).

Since $\Phi\left(f_{0}\right) \in \widehat{H}^{1}(\mathbb{S})$, it follows that (5.7) can hold only if $\Theta>0$. We also note that (5.6) ensures that the set $\mathcal{O}$ of all initial data that satisfy the Rayleigh-Taylor condition (5.7), that is

$$
\mathcal{O}=\left\{f_{0} \in H^{2}(\mathbb{S}): c_{\Theta}+a_{\mu} \Phi\left(f_{0}\right)>0\right\}
$$

is an open subset of $H^{2}(\mathbb{S})$ which is nonempty as it contains for example all constant functions.

In the following we fix an arbitrary $f_{0} \in \mathcal{O}$ and prove the generator property (5.2) for the operator

$$
\partial \Phi\left(f_{0}\right)[f]=\partial \mathbb{B}\left(f_{0}\right)[f]\left[\bar{\omega}_{0}\right]+\mathbb{B}\left(f_{0}\right)\left[\partial \bar{\omega}\left(f_{0}\right)[f]\right],
$$

where

$$
\bar{\omega}_{0}:=\bar{\omega}\left(f_{0}\right)
$$

is defined in Proposition 5.1. In view of (5.3) and of Proposition 5.1, we determine $\partial \bar{\omega}\left(f_{0}\right)[f]$ as the solution to the equation

$$
\left(1+a_{\mu} \mathbb{A}\left(f_{0}\right)\right)\left[\partial \bar{\omega}\left(f_{0}\right)[f]\right]=-c_{\Theta} f^{\prime}-a_{\mu} \partial \mathbb{A}\left(f_{0}\right)[f]\left[\bar{\omega}_{0}\right],
$$

where, combining the Lemmas 3.2 and A.1 $(i)$, we get

$$
\begin{aligned}
\partial \mathbb{A}\left(f_{0}\right)[f]\left[\bar{\omega}_{0}\right]= & -f^{\prime} \mathbb{B}_{2}\left(f_{0}\right)\left[\bar{\omega}_{0}\right]-f_{0}^{\prime} \partial \mathbb{B}_{2}\left(f_{0}\right)[f]\left[\bar{\omega}_{0}\right]-\partial \mathbb{B}_{1}\left(f_{0}\right)[f]\left[\bar{\omega}_{0}\right] \\
& +\pi^{-1}\left[f^{\prime} C_{0,1}\left(f_{0}\right)\left[\bar{\omega}_{0}\right]-2 f_{0}^{\prime} C_{2,2}\left(f_{0}, f_{0}\right)\left[f, f_{0}, \bar{\omega}_{0}\right]-C_{1,1}\left(f_{0}\right)\left[f, \bar{\omega}_{0}\right]\right. \\
& \left.+2 C_{3,2}\left(f_{0}, f_{0}\right)\left[f, f_{0}, f_{0}, \bar{\omega}_{0}\right]\right], \quad f \in H^{2}(\mathbb{S}) .
\end{aligned}
$$

Establishing (5.2) is now more difficult than for the Muskat problem with surface tension, because there are several leading order terms to be considered when dealing with $\partial \Phi\left(f_{0}\right)$, see the proof of Theorem 5.2. 
Besides, the Rayleigh-Taylor condition (5.7) does not appear in a natural way in the analysis and it has to be artificially built in instead. Indeed, let us first conclude from the Lemmas 3.1 and A.1 that

$$
\begin{aligned}
\partial \mathbb{B}\left(f_{0}\right)[f]\left[\bar{\omega}_{0}\right]= & f^{\prime} \mathbb{B}_{1}\left(f_{0}\right)\left[\bar{\omega}_{0}\right]+f_{0}^{\prime} \partial \mathbb{B}_{1}\left(f_{0}\right)[f]\left[\bar{\omega}_{0}\right]-\partial \mathbb{B}_{2}\left(f_{0}\right)[f]\left[\bar{\omega}_{0}\right] \\
& -2 \pi^{-1} C_{2,2}\left(f_{0}, f_{0}\right)\left[f, f_{0}, \bar{\omega}_{0}\right]+\pi^{-1} f^{\prime} C_{1,1}\left(f_{0}\right)\left[f_{0}, \bar{\omega}_{0}\right] \\
& +\pi^{-1} f_{0}^{\prime} C_{1,1}\left(f_{0}\right)\left[f, \bar{\omega}_{0}\right]-2 \pi^{-1} f_{0}^{\prime} C_{3,2}\left(f_{0}, f_{0}\right)\left[f, f_{0}, f_{0}, \bar{\omega}_{0}\right],
\end{aligned}
$$

and let

$$
[\tau \mapsto \Psi(\tau)]:[0,1] \rightarrow \mathcal{L}\left(H^{2}(\mathbb{S}), \widehat{H}^{1}(\mathbb{S})\right),
$$

denote the continuous path defined by

$$
\Psi(\tau)[f]:=\tau \partial \mathbb{B}\left(f_{0}\right)[f]\left[\bar{\omega}_{0}\right]+\mathbb{B}\left(\tau f_{0}\right)[w(\tau)[f]],
$$

where

$$
\begin{aligned}
w(\tau)[f]:= & -\left(1+a_{\mu} \mathbb{A}\left(\tau f_{0}\right)\right)^{-1}\left[c_{\Theta} f^{\prime}+\tau a_{\mu} \partial \mathbb{A}\left(f_{0}\right)[f]\left[\bar{\omega}_{0}\right]\right. \\
& \left.+(1-\tau) a_{\mu}\left(f^{\prime} \Phi\left(f_{0}\right)-\left\langle f^{\prime} \Phi\left(f_{0}\right)\right\rangle\right)\right] .
\end{aligned}
$$

The function defined in $(5.12)$ is related to $\partial \bar{\omega}\left(f_{0}\right)[f]$. We emphasize that the last term on the right hand side of (5.12) has been introduced artificially with the purpose of identifying the function $a_{\mathrm{RT}}$ when setting $\tau=0$, but also when relating $\Psi(\tau)$ locally to certain Fourier multipliers, see Theorem 5.2 below. If $\tau=1$, it follows that $\Psi(1)=\partial \Phi\left(f_{0}\right)$, while for $\tau=0$ we get

$$
\Psi(0)[f]=\mathbb{B}(0)[w(0)[f]]=-H\left[f^{\prime} a_{\mathrm{RT}}-\left\langle f^{\prime} a_{\mathrm{RT}}\right\rangle\right]=-H\left[f^{\prime} a_{\mathrm{RT}}\right],
$$

where we used once more the relation $\mathbb{B}(0)=H$. We note that, since $a_{\mathrm{RT}}$ is in general not constant, the operator $\Psi(0)$ is in general not a Fourier multiplier. However, we may benefit from the simpler structure of $\Psi(0)$, compared to that of $\partial \Phi\left(f_{0}\right)$, and the fact that the Rayleigh-Taylor condition holds to show that large real numbers belong to the spectrum of $\Psi(0)$, see Proposition 5.3.

We now derive some estimates for the operator $w \in C\left([0,1], \mathcal{L}\left(H^{2}(\mathbb{S}), \widehat{H}^{1}(\mathbb{S})\right)\right)$, which are needed later on in the analysis. Let therefore $\tau^{\prime} \in(1 / 2,1)$. Since $\Phi\left(f_{0}\right) \in H^{1}(\mathbb{S})$, it follows from Theorem 3.3 and (3.15) (with $r=1+\tau^{\prime}$ ) there exists a constant $C>0$ such that

$$
\|w(\tau)[f]\|_{2} \leq C\|f\|_{H^{1+\tau^{\prime}}}
$$

for all $f \in H^{2}(\mathbb{S})$ and $\tau \in[0,1]$. Furthermore, Theorem 3.5 and (3.15) show that additionally

$$
\|w(\tau)[f]\|_{H^{1}} \leq C\|f\|_{H^{2}} .
$$

Using the interpolation property (3.30), we conclude from (5.14), (5.15) that

$$
\|w(\tau)[f]\|_{H^{\tau^{\prime}}} \leq C\|f\|_{H^{1+2 \tau^{\prime}-\tau^{\prime 2}}}
$$

for all $f \in H^{2}(\mathbb{S})$ and $\tau \in[0,1]$.

The following result is the main step towards proving the generator property $(5.2)$. Below $\left(-\partial_{x}^{2}\right)^{1 / 2}$ stands for the Fourier multiplier with symbol $(|k|)_{k \in \mathbb{Z}}$, and the following identity is used

$$
\left(-\partial_{x}^{2}\right)^{1 / 2}[f]=H\left[f^{\prime}\right]=\mathbb{B}(0)\left[f^{\prime}\right] \quad \text { for all } f \in H^{1}(\mathbb{S}) .
$$

Theorem 5.2. Let $f_{0} \in H^{2}(\mathbb{S})$ and $\mu>0$ be given. Then, there exist $p \geq 3$, a p-partition of unity $\left\{\pi_{j}^{p}: 1 \leq j \leq 2^{p+1}\right\}$, a constant $K=K(p)$, and for each $j \in\left\{1, \ldots, 2^{p+1}\right\}$ and $\tau \in[0,1]$ there exist operators

$$
\mathbb{A}_{j, \tau} \in \mathcal{L}\left(H^{2}(\mathbb{S}), H^{1}(\mathbb{S})\right)
$$

such that

$$
\left\|\pi_{j}^{p} \Psi(\tau)[f]-\mathbb{A}_{j, \tau}\left[\pi_{j}^{p} f\right]\right\|_{H^{1}} \leq \mu\left\|\pi_{j}^{p} f\right\|_{H^{2}}+K\|f\|_{H^{31 / 16}}
$$


for all $j \in\left\{1, \ldots, 2^{p+1}\right\}, \tau \in[0,1]$, and $f \in H^{2}(\mathbb{S})$. The operator $\mathbb{A}_{j, \tau}$ is defined by

$$
\mathbb{A}_{j, \tau}:=-\alpha_{\tau}\left(x_{j}^{p}\right)\left(-\partial_{x}^{2}\right)^{1 / 2}+\beta_{\tau}\left(x_{j}^{p}\right) \partial_{x},
$$

where $x_{j}^{p} \in I_{j}^{p}$ is arbitrary, but fixed, and where

$$
\alpha_{\tau}:=\frac{1+(1-\tau) f_{0}^{\prime 2}}{1+f_{0}^{\prime 2}} a_{R T} \quad \text { and } \quad \beta_{\tau}:=\tau\left(\mathbb{B}_{1}\left(f_{0}\right)\left[\bar{\omega}_{0}\right]+\pi^{-1} C_{1,1}\left(f_{0}\right)\left[f_{0}, \bar{\omega}_{0}\right]+a_{\mu} \frac{\bar{\omega}_{0}}{1+\tau^{2} f_{0}^{\prime 2}}\right) .
$$

Proof. Let $p \geq 3$ be an integer which we fix later on in this proof and let $\left\{\pi_{j}^{p}: 1 \leq j \leq 2^{p+1}\right\}$ be a $p$-partition of unity, respectively let $\left\{\chi_{j}^{p}: 1 \leq j \leq 2^{p+1}\right\}$ be a family associated to this partition. We denote by $C$ constants which are independent of $p \in \mathbb{N}, f \in H^{2}(\mathbb{S}), \tau \in[0,1]$, and $j \in\left\{1, \ldots, 2^{p+1}\right\}$, while the constants denoted by $K$ may depend only upon $p$.

The lower order terms. We first note that

$$
\begin{aligned}
\left\|\pi_{j}^{p} \Psi(\tau)[f]-\mathbb{A}_{j, \tau}\left[\pi_{j}^{p} f\right]\right\|_{H^{1}} \leq & \left\|\pi_{j}^{p} \Psi(\tau)[f]-\mathbb{A}_{j, \tau}\left[\pi_{j}^{p} f\right]\right\|_{2}+\left\|\left(\pi_{j}^{p} \Psi(\tau)[f]-\mathbb{A}_{j, \tau}\left[\pi_{j}^{p} f\right]\right)^{\prime}\right\|_{2} \\
\leq & \left\|\pi_{j}^{p} \Psi(\tau)[f]-\mathbb{A}_{j, \tau}\left[\pi_{j}^{p} f\right]\right\|_{2}+\left\|\left(\pi_{j}^{p}\right)^{\prime} \Psi(\tau)[f]\right\|_{2} \\
& +\left\|\pi_{j}^{p}(\Psi(\tau)[f])^{\prime}-\mathbb{A}_{j, \tau}\left[\left(\pi_{j}^{p} f\right)^{\prime}\right]\right\|_{2} .
\end{aligned}
$$

The relations (3.6) (with $r=7 / 4$ ) and (5.14) (with $\tau^{\prime}=3 / 4$ ) yield

$$
\left\|\pi_{j}^{p} \Psi(\tau)[f]\right\|_{2}+\left\|\left(\pi_{j}^{p}\right)^{\prime} \Psi(\tau)[f]\right\|_{2} \leq K\|\Psi(\tau)[f]\|_{2} \leq K\|f\|_{H^{7 / 4}},
$$

and since $\max _{\tau \in[0,1]}\left(\left\|\alpha_{\tau}\right\|_{H^{1}}+\left\|\beta_{\tau}\right\|_{H^{1}}\right) \leq C$, it also holds that

$$
\left\|\mathbb{A}_{j, \tau}\left[\pi_{j}^{p} f\right]\right\|_{2} \leq K\|f\|_{H^{1}} .
$$

Therewith we get

$$
\left\|\pi_{j}^{p} \Psi(\tau)[f]-\mathbb{A}_{j, \tau}\left[\pi_{j}^{p} f\right]\right\|_{H^{1}} \leq\left\|\pi_{j}^{p}(\Psi(\tau)[f])^{\prime}-\mathbb{A}_{j, \tau}\left[\left(\pi_{j}^{p} f\right)^{\prime}\right]\right\|_{2}+K\|f\|_{H^{7 / 4}} .
$$

Moreover, combining (5.11), (3.10) (with $r=7 / 4$ and $\tau=3 / 4$ ), Lemma A.1 (ii) (with $\tau=3 / 4$ and $r=15 / 8$ ), and (5.16) (with $\tau^{\prime}=3 / 4$ ), we may write

$$
\begin{aligned}
(\Psi(\tau)[f])^{\prime}= & \mathbb{B}_{3}\left(\tau f_{0}\right)\left[(w(\tau)[f])^{\prime}\right]+\tau f^{\prime \prime}\left(\mathbb{B}_{1}\left(f_{0}\right)\left[\bar{\omega}_{0}\right]+\pi^{-1} C_{1,1}\left(f_{0}\right)\left[f_{0}, \bar{\omega}_{0}\right]\right)+\tau \pi^{-1} f_{0}^{\prime} C_{0,1}\left(f_{0}\right)\left[\left(f^{\prime} \bar{\omega}_{0}\right)^{\prime}\right] \\
& -2 \tau \pi^{-1} C_{1,2}\left(f_{0}, f_{0}\right)\left[f_{0},\left(f^{\prime} \bar{\omega}_{0}\right)^{\prime}\right]-2 \tau \pi^{-1} f_{0}^{\prime} C_{2,2}\left(f_{0}, f_{0}\right)\left[f_{0}, f_{0},\left(f^{\prime} \bar{\omega}_{0}\right)^{\prime}\right]+T_{\mathrm{lot}}^{\Psi, \tau}[f],
\end{aligned}
$$

where

$$
\left\|T_{\mathrm{lot}}^{\Psi, \tau}[f]\right\|_{2} \leq C\|f\|_{H^{31 / 16}} .
$$

Consequently, we are left to estimate the $L_{2}$-norm of the difference

$$
\begin{gathered}
\pi_{j}^{p} \mathbb{B}_{3}\left(\tau f_{0}\right)\left[(w(\tau)[f])^{\prime}\right]+\tau \pi_{j}^{p} f^{\prime \prime}\left(\mathbb{B}_{1}\left(f_{0}\right)\left[\bar{\omega}_{0}\right]+\pi^{-1} C_{1,1}\left(f_{0}\right)\left[f_{0}, \bar{\omega}_{0}\right]\right)+\tau \pi^{-1} \pi_{j}^{p} f_{0}^{\prime} C_{0,1}\left(f_{0}\right)\left[\left(f^{\prime} \bar{\omega}_{0}\right)^{\prime}\right] \\
-2 \tau \pi^{-1} \pi_{j}^{p} C_{1,2}\left(f_{0}, f_{0}\right)\left[f_{0},\left(f^{\prime} \bar{\omega}_{0}\right)^{\prime}\right]-2 \tau \pi^{-1} \pi_{j}^{p} f_{0}^{\prime} C_{2,2}\left(f_{0}, f_{0}\right)\left[f_{0}, f_{0},\left(f^{\prime} \bar{\omega}_{0}\right)^{\prime}\right]-\mathbb{A}_{j, \tau}\left[\left(\pi_{j}^{p} f\right)^{\prime}\right] .
\end{gathered}
$$

Higher order terms $I$. Given $1 \leq j \leq 2^{p+1}$, we set

$$
\mathbb{A}_{j, \tau}^{1}:=\left(\mathbb{B}_{1}\left(f_{0}\right)\left[\bar{\omega}_{0}\right]+\pi^{-1} C_{1,1}\left(f_{0}\right)\left[f_{0}, \bar{\omega}_{0}\right]\right)\left(x_{j}^{p}\right) \partial_{x} .
$$

Since $\mathbb{B}_{1}\left(f_{0}\right)\left[\bar{\omega}_{0}\right], C_{1,1}\left(f_{0}\right)\left[f_{0}, \bar{\omega}_{0}\right] \in H^{1}(\mathbb{S}) \hookrightarrow \mathrm{C}^{1 / 2}(\mathbb{S})$ and $\chi_{j}^{p} \pi_{j}^{p}=\pi_{j}^{p}$, it follows that

$$
\begin{aligned}
\| \pi_{j}^{p} & f^{\prime \prime}\left(\mathbb{B}_{1}\left(f_{0}\right)\left[\bar{\omega}_{0}\right]+\pi^{-1} C_{1,1}\left(f_{0}\right)\left[f_{0}, \bar{\omega}_{0}\right]\right)-\mathbb{A}_{j, \tau}^{1}\left[\left(\pi_{j}^{p} f\right)^{\prime}\right] \|_{2} \\
\leq & K\|f\|_{H^{1}}+\left\|\chi_{j}^{p}\left(\mathbb{B}_{1}\left(f_{0}\right)\left[\bar{\omega}_{0}\right]-\mathbb{B}_{1}\left(f_{0}\right)\left[\bar{\omega}_{0}\right]\left(x_{j}^{p}\right)\right)\right\|_{\infty}\left\|\pi_{j}^{p} f\right\|_{H^{2}} \\
& +\left\|\chi_{j}^{p}\left(C_{1,1}\left(f_{0}\right)\left[f_{0}, \bar{\omega}_{0}\right]-C_{1,1}\left(f_{0}\right)\left[f_{0}, \bar{\omega}_{0}\right]\left(x_{j}^{p}\right)\right)\right\|_{\infty}\left\|\pi_{j}^{p} f\right\|_{H^{2}} \\
\leq & \frac{\mu}{4}\left\|\pi_{j}^{p} f\right\|_{H^{2}}+K\|f\|_{H^{1}},
\end{aligned}
$$

provided that $p$ is sufficiently large. 
Higher order terms II. Letting

$$
\mathbb{A}_{j, \tau}^{2}:=\frac{\bar{\omega}_{0}\left(x_{j}^{p}\right) f_{0}^{\prime}\left(x_{j}^{p}\right)}{\left(1+f_{0}^{\prime 2}\left(x_{j}^{p}\right)\right)^{2}}\left(-\partial_{x}^{2}\right)^{-1 / 2},
$$

it holds that

$$
\pi_{j}^{p} C_{1,2}\left(f_{0}, f_{0}\right)\left[f_{0},\left(f^{\prime} \bar{\omega}_{0}\right)^{\prime}\right]-\pi \mathbb{A}_{j, \tau}^{2}\left[\left(\pi_{j}^{p} f\right)^{\prime}\right]=T_{1}[f]+T_{2}[f]+T_{3}[f]
$$

where

$$
\begin{aligned}
& T_{1}[f]=\pi_{j}^{p} C_{1,2}\left(f_{0}, f_{0}\right)\left[f_{0},\left(f^{\prime} \bar{\omega}_{0}\right)^{\prime}\right]-C_{1,2}\left(f_{0}, f_{0}\right)\left[f_{0}, \pi_{j}^{p}\left(f^{\prime} \bar{\omega}_{0}\right)^{\prime}\right], \\
& T_{2}[f]=C_{1,2}\left(f_{0}, f_{0}\right)\left[f_{0}, \pi_{j}^{p}\left(f^{\prime} \bar{\omega}_{0}\right)^{\prime}\right]-\frac{f_{0}^{\prime}\left(x_{j}^{p}\right)}{\left(1+f_{0}^{\prime 2}\left(x_{j}^{p}\right)\right)^{2}} C_{0,0}\left[\pi_{j}^{p}\left(f^{\prime} \bar{\omega}_{0}\right)^{\prime}\right], \\
& T_{3}[f]=\frac{f_{0}^{\prime}\left(x_{j}^{p}\right)}{\left(1+f_{0}^{\prime 2}\left(x_{j}^{p}\right)\right)^{2}} C_{0,0}\left[\pi_{j}^{p}\left(f^{\prime} \bar{\omega}_{0}\right)^{\prime}\right]-\pi \mathbb{A}_{j, \tau}^{2}\left[\left(\pi_{j}^{p} f\right)^{\prime}\right] .
\end{aligned}
$$

The first term may be estimated, by using integration by parts, in a similar way as the term $T_{11}[h]$ in the proof of Theorem 4.2, that is

$$
\left\|T_{1}[f]\right\|_{2} \leq K\left\|f^{\prime} \bar{\omega}_{0}\right\|_{2} \leq K\|f\|_{H^{1}} .
$$

Besides, the same arguments used to derive (4.21) show that for $p$ sufficiently large

$$
\left\|T_{2}[f]\right\|_{2} \leq \frac{\mu}{16}\left\|\pi_{j}^{p} f\right\|_{H^{2}}+K\|f\|_{H^{1}} .
$$

Finally, it holds that

$$
\begin{aligned}
\left\|T_{3}[f]\right\|_{2} \leq & \left\|C_{0,0}\left[\left(\pi_{j} f\right)^{\prime \prime}\left(\bar{\omega}_{0}-\bar{\omega}_{0}\left(x_{j}^{p}\right)\right)\right]\right\|_{2}+\left\|C_{0,0}\left[\pi_{j}^{p} f^{\prime} \bar{\omega}_{0}^{\prime}\right]\right\|_{2}+\left\|C_{0,0}\left[\left(\left(\pi_{j}^{p}\right)^{\prime \prime} f+2\left(\pi_{j}^{p}\right)^{\prime} f^{\prime}\right) \bar{\omega}_{0}\right]\right\|_{2} \\
& +\left\|\bar{\omega}_{0}\right\|_{\infty}\left\|\int_{-\pi}^{\pi}\left[\frac{1}{t_{[s]}}-\frac{1}{s / 2}\right]\left(\pi_{j} f\right)^{\prime \prime}(\cdot-s) \mathrm{d} s\right\|_{2},
\end{aligned}
$$

and, recalling that $\chi_{j}^{p}=1$ on $\operatorname{supp} \pi_{j}^{p}$, we obtain, by using integration by parts, Lemma A.1 $(i)$, and the fact that $\bar{\omega}_{0} \in H^{1}(\mathbb{S}) \hookrightarrow \mathrm{C}^{1 / 2}(\mathbb{S})$ the estimate

$$
\begin{aligned}
\left\|T_{3}[f]\right\|_{2} & \leq\left\|C_{0,0}\left[\left(\pi_{j} f\right)^{\prime \prime} \chi_{j}^{p}\left(\bar{\omega}_{0}-\bar{\omega}_{0}\left(x_{j}^{p}\right)\right)\right]\right\|_{2}+K\|f\|_{H^{1}} \leq C\left\|\pi_{j} f\right\|_{H^{2}}\left\|\chi_{j}^{p}\left(\bar{\omega}_{0}-\bar{\omega}_{0}\left(x_{j}^{p}\right)\right)\right\|_{\infty}+K\|f\|_{H^{1}} \\
& \leq \frac{\mu}{16}\left\|\pi_{j}^{p} f\right\|_{H^{2}}+K\|f\|_{H^{1}},
\end{aligned}
$$

provided $p$ is sufficiently large. Summarizing, we have shown that

$$
2\left\|\pi_{j}^{p} C_{1,2}\left(f_{0}, f_{0}\right)\left[f_{0},\left(f^{\prime} \bar{\omega}_{0}\right)^{\prime}\right]-\pi \mathbb{A}_{j, \tau}^{2}\left[\left(\pi_{j}^{p} f\right)^{\prime}\right]\right\|_{2} \leq \frac{\mu}{4}\left\|\pi_{j}^{p} f\right\|_{H^{2}}+K\|f\|_{H^{1}}
$$

and similarly we get

$$
\begin{aligned}
& \left\|\pi_{j}^{p} f_{0}^{\prime} C_{0,1}\left(f_{0}\right)\left[\left(f^{\prime} \bar{\omega}_{0}\right)^{\prime}\right]-\pi\left(1+f_{0}^{\prime 2}\left(x_{j}^{p}\right)\right) \mathbb{A}_{j, \tau}^{2}\left[\left(\pi_{j}^{p} f\right)^{\prime}\right]\right\|_{2} \\
& \quad+2\left\|\pi_{j}^{p} f_{0}^{\prime} C_{2,2}\left(f_{0}, f_{0}\right)\left[f_{0}, f_{0}\left(f^{\prime} \bar{\omega}_{0}\right)^{\prime}\right]-\pi f_{0}^{\prime 2}\left(x_{j}^{p}\right) \mathbb{A}_{j, \tau}^{2}\left[\left(\pi_{j}^{p} f\right)^{\prime}\right]\right\|_{2} \\
& \quad \leq \frac{\mu}{4}\left\|\pi_{j}^{p} f\right\|_{H^{2}}+K\|f\|_{H^{1}} .
\end{aligned}
$$

Higher order terms III. We are left to consider the function

$$
\pi_{j}^{p} \mathbb{B}_{3}\left(\tau f_{0}\right)\left[(w(\tau)[f])^{\prime}\right]=\pi^{-1} \pi_{j}^{p}\left(C_{0,1}\left(f_{\tau_{0}}\right)\left[w^{\prime}\right]+f_{\tau_{0}}^{\prime} C_{1,1}\left(f_{\tau_{0}}\right)\left[f_{\tau_{0}}, w^{\prime}\right]\right),
$$

where, for the sake of brevity, we have set

$$
f_{\tau_{0}}:=\tau f_{0} \quad \text { and } \quad w:=w(\tau)[f] .
$$

Let further

$$
\phi_{\tau}:=a_{\mathrm{RT}}-\tau a_{\mu} f_{0}^{\prime}\left(\mathbb{B}_{1}\left(f_{0}\right)\left[\bar{\omega}_{0}\right]+\pi^{-1} C_{1,1}\left(f_{0}\right)\left[f_{0}, \bar{\omega}_{0}\right]\right) \in H^{1}(\mathbb{S}) .
$$


We first derive an estimate for the $L_{2}$-norm of $\pi_{j}^{p} w^{\prime}$. To this end we differentiate (5.12) once to obtain, in view of (3.27), (5.10), and Lemma A.1 (i)-(ii), that

$$
\begin{aligned}
\left(1+a_{\mu} \mathbb{A}\left(f_{\tau_{0}}\right)\right)\left[\left(\pi_{j}^{p} w\right)^{\prime}\right]= & -\phi_{\tau} \pi_{j}^{p} f^{\prime \prime}+T_{\mathrm{lot}}^{w, j, \tau}[f]+\tau a_{\mu} \pi^{-1}\left(2 f_{0}^{\prime} C_{1,2}\left(f_{0}, f_{0}\right)\left[f_{0}, \pi_{j}^{p}\left(f^{\prime} \bar{\omega}_{0}\right)^{\prime}\right]\right. \\
& \left.+C_{0,1}\left(f_{0}\right)\left[\pi_{j}^{p}\left(f^{\prime} \bar{\omega}_{0}\right)^{\prime}\right]-2 C_{2,2}\left(f_{0}, f_{0}\right)\left[f_{0}, f_{0}, \pi_{j}^{p}\left(f^{\prime} \bar{\omega}_{0}\right)^{\prime}\right]\right) .
\end{aligned}
$$

Combining (3.10) (with $\tau=3 / 4$ and $r=7 / 4$ ), (3.29) (with $\tau=3 / 4$ ), (4.17), (5.14) and (5.16) (both with $\tau^{\prime}=3 / 4$ ), and Lemma A.1 $(i)-(i i)$ (with $\tau=3 / 4$ and $r=15 / 8$ ) we get that

$$
\left\|T_{\mathrm{lot}}^{w, j, \tau}[f]\right\|_{2} \leq K\|f\|_{H^{31 / 16}} .
$$

The relation (5.23) together with Theorem 3.3, Lemma A.1 (i), and (5.14) (with $\tau^{\prime}=3 / 4$ ) now yields

$$
\left\|\pi_{j}^{p} w^{\prime}\right\|_{2} \leq\left\|\left(\pi_{j}^{p} w\right)^{\prime}\right\|_{2}+\left\|\left(\pi_{j}^{p}\right)^{\prime} w\right\|_{2} \leq C\left\|\pi_{j}^{p} f\right\|_{H^{2}}+K\|f\|_{H^{31 / 16}}
$$

We now consider the second term on the right hand side of (5.22). Letting

$$
\mathbb{A}_{j, \tau}^{3}:=\frac{f_{\tau_{0}}^{\prime 2}\left(x_{j}^{p}\right)}{1+f_{\tau_{0}}^{\prime 2}\left(x_{j}^{p}\right)}\left[-\phi_{\tau}\left(x_{j}^{p}\right)\left(-\partial_{x}^{2}\right)^{1 / 2}-\tau a_{\mu} \frac{\bar{\omega}_{0}\left(x_{j}^{p}\right)}{1+f_{\tau_{0}}^{\prime 2}\left(x_{j}^{p}\right)} \partial_{x}\right],
$$

we write

$$
\pi_{j}^{p} f_{\tau_{0}}^{\prime} C_{1,1}\left(f_{\tau_{0}}\right)\left[f_{\tau_{0}}, w^{\prime}\right]-\pi \mathbb{A}_{j, \tau}^{3}\left[\left(\pi_{j}^{p} f\right)^{\prime}\right]=T_{4}[f]+T_{5}[f]+T_{6}[f],
$$

where

$$
\begin{aligned}
& T_{4}[f]=\pi_{j}^{p} f_{\tau_{0}}^{\prime} C_{1,1}\left(f_{\tau_{0}}\right)\left[f_{\tau_{0}}, w^{\prime}\right]-f_{\tau_{0}}^{\prime}\left(x_{j}^{p}\right) C_{1,1}\left(f_{\tau_{0}}\right)\left[f_{\tau_{0}}, \pi_{j}^{p} w^{\prime}\right], \\
& T_{5}[f]=f_{\tau_{0}}^{\prime}\left(x_{j}^{p}\right) C_{1,1}\left(f_{\tau_{0}}\right)\left[f_{\tau_{0}}, \pi_{j}^{p} w^{\prime}\right]-\frac{f_{\tau_{0}}^{\prime 2}\left(x_{j}^{p}\right)}{1+f_{\tau_{0}}^{\prime 2}\left(x_{j}^{p}\right)} C_{0,0}\left[\pi_{j}^{p} w^{\prime}\right], \\
& T_{6}[f]=\frac{f_{\tau_{0}}^{\prime 2}\left(x_{j}^{p}\right)}{1+f_{\tau_{0}}^{\prime 2}\left(x_{j}^{p}\right)} C_{0,0}\left[\pi_{j}^{p} w^{\prime}\right]-\pi \mathbb{A}_{j, \tau}^{3}\left[\left(\pi_{j}^{p} f\right)^{\prime}\right] .
\end{aligned}
$$

The arguments that led to (4.19) together with (5.25) show that

$$
\left\|T_{4}[f]\right\|_{2} \leq \frac{\mu}{24}\left\|\pi_{j}^{p} f\right\|_{H^{2}}+K\|f\|_{H^{31 / 16}},
$$

provided that $p$ is sufficiently large, while arguing as in the derivation of (4.21) we obtain that

$$
\left\|T_{5}[f]\right\|_{2} \leq \frac{\mu}{24}\left\|\pi_{j}^{p} f\right\|_{H^{2}}+K\|f\|_{H^{31 / 16}} .
$$

Concerning $T_{6}[f]$, we find, by using fact that the Hilbert transform satisfies $H^{2}=-\mathrm{id}_{L_{2}(\mathbb{S})}$, the following relation

$$
\left\|T_{6}[f]\right\|_{2} \leq\left\|C_{0,0}\left[\pi_{j}^{p} w^{\prime}\right]+\pi \phi_{\tau}\left(x_{j}^{p}\right) H\left[\left(\pi_{j}^{p} f\right)^{\prime \prime}\right]-\tau a_{\mu} \pi \frac{\bar{\omega}_{0}\left(x_{j}^{p}\right)}{1+f_{\tau_{0}}^{\prime 2}\left(x_{j}^{p}\right)} H^{2}\left[\left(\pi_{j}^{p} f\right)^{\prime \prime}\right]\right\|_{2},
$$

and, since integration by parts and (5.14) (with $\tau^{\prime}=3 / 4$ ) yield

$$
\left\|C_{0,0}\left[\pi_{j}^{p} w^{\prime}\right]-\pi H\left[\pi_{j}^{p} w^{\prime}\right]\right\|_{2}+\left\|C_{0,0}\left[\pi_{j}^{p} f^{\prime \prime}\right]-\pi H\left[\left(\pi_{j}^{p} f\right)^{\prime \prime}\right]\right\|_{2} \leq K\|w\|_{2} \leq K\|f\|_{H^{7 / 4}},
$$

we conclude that

$$
\begin{aligned}
\left\|T_{6}[f]\right\|_{2} & \leq\left\|\pi_{j}^{p} w^{\prime}+\phi_{\tau}\left(x_{j}^{p}\right)\left(\pi_{j}^{p} f\right)^{\prime \prime}-\tau a_{\mu} \frac{\bar{\omega}_{0}\left(x_{j}^{p}\right)}{1+f_{\tau_{0}}^{\prime 2}\left(x_{j}^{p}\right)} H\left[\left(\pi_{j}^{p} f\right)^{\prime \prime}\right]\right\|_{2}+K\|f\|_{H^{7 / 4}} \\
& \leq\left\|\pi_{j}^{p} w^{\prime}+\phi_{\tau}\left(x_{j}^{p}\right) \pi_{j}^{p} f^{\prime \prime}-\frac{\tau a_{\mu}}{\pi} \frac{\bar{\omega}_{0}\left(x_{j}^{p}\right)}{1+f_{\tau_{0}}^{\prime 2}\left(x_{j}^{p}\right)} C_{0,0}\left[\pi_{j}^{p} f^{\prime \prime}\right]\right\|_{2}+K\|f\|_{H^{7 / 4}} .
\end{aligned}
$$

Combining (3.16) and (5.23), we further get

$$
\left\|\pi_{j}^{p} w^{\prime}+\phi_{\tau}\left(x_{j}^{p}\right) \pi_{j}^{p} f^{\prime \prime}-\frac{\tau a_{\mu}}{\pi} \frac{\bar{\omega}_{0}\left(x_{j}^{p}\right)}{1+f_{\tau_{0}}^{\prime 2}\left(x_{j}^{p}\right)} C_{0,0}\left[\pi_{j}^{p} f^{\prime \prime}\right]\right\|_{2}
$$




$$
\begin{aligned}
\leq & \left\|\chi_{j}^{p}\left(\phi_{\tau}-\phi_{\tau}\left(x_{j}^{p}\right)\right)\right\|_{\infty}\left\|\pi_{j}^{p} f^{\prime \prime}\right\|_{2}+\|\left(1+a_{\mu} \mathbb{A}\left(f_{\tau_{0}}\right)\left[\left(\pi_{j}^{p}\right)^{\prime} w\right] \|_{2}\right. \\
& +\left\|f_{0}^{\prime} C_{1,2}\left(f_{0}, f_{0}\right)\left[f_{0}, \pi_{j}^{p}\left(f^{\prime} \bar{\omega}_{0}\right)^{\prime}\right]-\frac{\bar{\omega}_{0}\left(x_{j}^{p}\right) f_{0}^{\prime 2}\left(x_{j}^{p}\right)}{\left(1+f_{0}^{\prime 2}\left(x_{j}^{p}\right)\right)^{2}} C_{0,0}\left[\pi_{j}^{p} f^{\prime \prime}\right]\right\|_{2} \\
& +\left\|C_{0,1}\left(f_{0}\right)\left[\pi_{j}^{p}\left(f^{\prime} \bar{\omega}_{0}\right)^{\prime}\right]-\frac{\bar{\omega}_{0}\left(x_{j}^{p}\right)}{1+f_{0}^{\prime 2}\left(x_{j}^{p}\right)} C_{0,0}\left[\pi_{j}^{p} f^{\prime \prime}\right]\right\|_{2} \\
& +\left\|C_{2,2}\left(f_{0}, f_{0}\right)\left[f_{0}, f_{0}, \pi_{j}^{p}\left(f^{\prime} \bar{\omega}_{0}\right)^{\prime}\right]-\frac{\bar{\omega}_{0}\left(x_{j}^{p}\right) f_{0}^{\prime 2}\left(x_{j}^{p}\right)}{\left(1+f_{0}^{\prime 2}\left(x_{j}^{p}\right)\right)^{2}} C_{0,0}\left[\pi_{j}^{p} f^{\prime \prime}\right]\right\|_{2} \\
& +\left\|T_{\mathrm{lot}}^{w, j, \tau}[f]\right\|_{2}+\left\|f_{\tau_{0}}^{\prime} \mathbb{B}_{2}\left(f_{\tau_{0}}\right)\left[\pi_{j}^{p} w^{\prime}\right]\right\|_{2}+\left\|\mathbb{B}_{1}\left(f_{\tau_{0}}\right)\left[\pi_{j}^{p} w^{\prime}\right]\right\|_{2} \\
& +\left\|f_{\tau_{0}}^{\prime} C_{0,1}\left(f_{\tau_{0}}\right)\left[\pi_{j}^{p} w^{\prime}\right]-\frac{f_{\tau_{0}}^{\prime}\left(x_{j}^{p}\right)}{1+f_{\tau_{0}}^{\prime 2}\left(x_{j}^{p}\right)} C_{0,0}\left[\pi_{j}^{p} w^{\prime}\right]\right\|_{2} \\
& +\left\|C_{1,1}\left(f_{\tau_{0}}\right)\left[f_{\tau_{0}}, \pi_{j}^{p} w^{\prime}\right]-\frac{f_{\tau_{0}}^{\prime}\left(x_{j}^{p}\right)}{1+f_{\tau_{0}}^{\prime 2}\left(x_{j}^{p}\right)} C_{0,0}\left[\pi_{j}^{p} w^{\prime}\right]\right\|_{2},
\end{aligned}
$$

and the estimates (4.17), (5.14) (with $\left.\tau^{\prime}=3 / 4\right),(5.24)$, together with the arguments used to estimate $\left\|T_{2}[f]\right\|_{2}$ show, for $p$ sufficiently large, that

$$
\left\|T_{6}[f]\right\|_{2} \leq \frac{\mu}{24}\left\|\pi_{j}^{p} f\right\|_{H^{2}}+K\|f\|_{H^{31 / 16}} .
$$

Altogether, we have shown that

$$
\left\|\pi_{j}^{p} f_{\tau_{0}}^{\prime} C_{1,1}\left(f_{\tau_{0}}\right)\left[f_{\tau_{0}}, w^{\prime}\right]-\pi \mathbb{A}_{j, \tau}^{3}\left[\left(\pi_{j}^{p} f\right)^{\prime}\right]\right\|_{2} \leq \frac{\mu}{8}\left\|\pi_{j}^{p} f\right\|_{H^{2}}+K\|f\|_{H^{31 / 16}} .
$$

Letting

$$
\mathbb{A}_{j, \tau}^{4}:=\frac{1}{1+f_{\tau_{0}}^{\prime 2}\left(x_{j}^{p}\right)}\left[-\phi_{\tau}\left(x_{j}^{p}\right)\left(-\partial_{x}^{2}\right)^{1 / 2}-\tau a_{\mu} \frac{\bar{\omega}_{0}\left(x_{j}^{p}\right)}{1+f_{\tau_{0}}^{\prime 2}\left(x_{j}^{p}\right)} \partial_{x}\right],
$$

we obtain in a similar way, that

$$
\left\|\pi_{j}^{p} C_{0,1}\left(f_{\tau_{0}}\right)\left[w^{\prime}\right]-\pi \mathbb{A}_{j, \tau}^{4}\left[\left(\pi_{j}^{p} f\right)^{\prime}\right]\right\|_{2} \leq \frac{\mu}{8}\left\|\pi_{j}^{p} f\right\|_{H^{2}}+K\|f\|_{H^{31 / 16}},
$$

provided that $p$ is sufficiently large, and therewith we conclude that

$$
\left\|\pi_{j}^{p} \mathbb{B}_{3}\left(\tau f_{0}\right)\left[(w(\tau)[f])^{\prime}\right]-\left(\mathbb{A}_{j, \tau}^{3}+\mathbb{A}_{j, \tau}^{4}\right)\left[\left(\pi_{j}^{p} f\right)^{\prime}\right]\right\|_{2} \leq \frac{\mu}{4}\left\|\pi_{j}^{p} f\right\|_{H^{2}}+K\|f\|_{H^{31 / 16}} .
$$

Final step. Using the identity $\bar{\omega}_{0}=-c_{\Theta} f_{0}^{\prime}-a_{\mu} \mathbb{A}\left(f_{0}\right)\left[\bar{\omega}_{0}\right]$, it is not difficult to see that

$$
\mathbb{A}_{j, \tau}=\tau\left[\mathbb{A}_{j, p}^{1}-2 \mathbb{A}_{j, p}^{2}+\left(1+f_{0}^{\prime 2}\left(x_{j}^{p}\right)\right) \mathbb{A}_{j, p}^{2}-2 f_{0}^{\prime 2}\left(x_{j}^{p}\right) \mathbb{A}_{j, p}^{2}\right]+\mathbb{A}_{j, p}^{3}+\mathbb{A}_{j, p}^{4},
$$

and (5.19), (5.20), (5.21), and (5.26) immediately yield (5.17).

Making use of the fact that for $f_{0} \in \mathcal{O}$ the Rayleigh-Taylor condition $a_{\mathrm{RT}}>0$ is satisfied, it follows from the general result in Proposition 5.3 below that $\Psi(0)$ contains in its resolvent set all sufficiently large real numbers.

Proposition 5.3. Let $a \in H^{1}(\mathbb{S})$ be a positive function. Then, there exists $\omega_{0} \geq 1$ with the property that $\lambda+H\left[a \partial_{x}\right] \in \operatorname{Isom}\left(H^{2}(\mathbb{S}), H^{1}(\mathbb{S})\right)$ for all $\lambda \in\left[\omega_{0}, \infty\right)$.

Proof. Let $m:=\min _{\mathbb{S}} a>0$. We introduce the continuous path $[\tau \mapsto B(\tau)]:[0,1] \rightarrow \mathcal{L}\left(H^{2}(\mathbb{S}), H^{1}(\mathbb{S})\right)$ via

$$
B(\tau):=H\left[a_{\tau} \partial_{x}\right] \quad \text { with } \quad a_{\tau}:=(1-\tau) m+\tau a \geq m .
$$

Since $\lambda+B(0)$ is the Fourier multiplier with symbol $(\lambda+m|k|)_{k \in \mathbb{Z}}$, it is obvious that $\lambda+B(0)$ is invertible for all $\lambda>0$. If $\lambda$ is sufficiently large, we show below that $\lambda+B(1)=\lambda+H\left[a \partial_{x}\right]$ has this property too. 
To this end we prove that for each $\mu>0$ there exists $p \geq 3$, a $p$-partition of unity $\left\{\pi_{j}^{p}: 1 \leq j \leq 2^{p+1}\right\}$, a constant $K=K(p)$, and for each $j \in\left\{1, \ldots, 2^{p+1}\right\}$ and $\tau \in[0,1]$ there exist operators

$$
\mathbb{B}_{j, \tau} \in \mathcal{L}\left(H^{2}(\mathbb{S}), H^{1}(\mathbb{S})\right)
$$

such that

$$
\left\|\pi_{j}^{p} B(\tau)[f]-\mathbb{B}_{j, \tau}\left[\pi_{j}^{p} f\right]\right\|_{H^{1}} \leq \mu\left\|\pi_{j}^{p} f\right\|_{H^{2}}+K\|f\|_{H^{7 / 4}}
$$

for all $j \in\left\{1, \ldots, 2^{p+1}\right\}, \tau \in[0,1]$, and $f \in H^{2}(\mathbb{S})$. The operators $\mathbb{B}_{j, \tau}$ are the Fourier multipliers

$$
\mathbb{B}_{j, \tau}:=a_{\tau}\left(x_{j}^{p}\right)\left(-\partial_{x}^{2}\right)^{1 / 2}
$$

with $x_{j}^{p} \in I_{j}^{p}$. Indeed, given $p \geq 3$, let $\left\{\pi_{j}^{p}: 1 \leq j \leq 2^{p+1}\right\}$ be a $p$-partition of unity and let $\left\{\chi_{j}^{p}: 1 \leq\right.$ $\left.j \leq 2^{p+1}\right\}$ be a family associated to this partition. Integrating by parts we get

$$
\begin{aligned}
\left\|\pi_{j}^{p} B(\tau)[f]-\mathbb{B}_{j, \tau}\left[\pi_{j}^{p} f\right]\right\|_{H^{1}} \leq & \left\|\pi_{j}^{p} B(\tau)[f]-\mathbb{B}_{j, \tau}\left[\pi_{j}^{p} f\right]\right\|_{2}+\left\|\left(\pi_{j}^{p}\right)^{\prime} B(\tau)[f]\right\|_{2}+\left\|\mathbb{B}_{j, \tau}\left[\left(\pi_{j}^{p}\right)^{\prime} f\right]\right\|_{2} \\
& +\left\|\pi_{j}^{p}(B(\tau)[f])^{\prime}-\mathbb{B}_{j, \tau}\left[\pi_{j}^{p} f^{\prime}\right]\right\|_{2} \\
\leq & K\|f\|_{H^{1}}+\left\|\pi_{j}^{p} H\left[\left(a_{\tau} f^{\prime}\right)^{\prime}\right]-a_{\tau}\left(x_{j}^{p}\right) H\left[\left(\pi_{j}^{p} f^{\prime}\right)^{\prime}\right]\right\|_{2} \\
\leq & K\|f\|_{H^{\tau / 4}}+\left\|\pi_{j}^{p} H\left[a_{\tau} f^{\prime \prime}\right]-a_{\tau}\left(x_{j}^{p}\right) H\left[\pi_{j}^{p} f^{\prime \prime}\right]\right\|_{2} \\
\leq & K\|f\|_{H^{7 / 4}}+\left\|\pi_{j}^{p} H\left[a_{\tau} f^{\prime \prime}\right]-H\left[a_{\tau} \pi_{j}^{p} f^{\prime \prime}\right]\right\|_{2}+\left\|H\left[\left(a_{\tau}-a_{\tau}\left(x_{j}^{p}\right)\right) \pi_{j}^{p} f^{\prime \prime}\right]\right\|_{2} \\
\leq & \left.K\|f\|_{H^{7 / 4}}+\left\|\left(a_{\tau}-a_{\tau}\left(x_{j}^{p}\right)\right) \chi_{j}^{p}\right\|_{\infty} \| \pi_{j}^{p} f^{\prime \prime}\right] \|_{2} \\
\leq & \mu\left\|\pi_{j}^{p} f\right\|_{H^{2}}+K\|f\|_{H^{7 / 4}}
\end{aligned}
$$

provided that $p$ is sufficiently large, and (5.27) follows.

A simple computation shows that there exists $\kappa \geq 1$ such that

$$
\kappa\left\|\left(\lambda+\alpha\left(-\partial_{x}^{2}\right)^{1 / 2}\right)[f]\right\|_{H^{1}} \geq \lambda \cdot\|f\|_{H^{1}}+\|f\|_{H^{2}}
$$

for all $f \in H^{2}(\mathbb{S}), \alpha \geq m$, and $\lambda \in[1, \infty)$. Set $\mu:=1 / 2 \kappa$ in (5.27). Since $a_{\tau} \geq m$, it follows from $(5.27)$ and (5.28) that

$$
\begin{aligned}
\kappa\left\|\pi_{j}^{p}(\lambda+B(\tau))[f]\right\|_{H^{1}} & \geq \kappa\left\|\left(\lambda+\mathbb{B}_{j, \tau}\right)\left[\pi_{j}^{p} f\right]\right\|_{H^{1}}-\kappa\left\|\pi_{j}^{p} B(\tau)[f]-\mathbb{B}_{j, \tau}\left[\pi_{j}^{p} f\right]\right\|_{H^{1}} \\
& \geq \lambda \cdot\left\|\pi_{j}^{p} f\right\|_{H^{1}}+\frac{1}{2}\left\|\pi_{j}^{p} f\right\|_{H^{2}}-\kappa K\|f\|_{H^{7 / 4}}
\end{aligned}
$$

for all $f \in H^{2}(\mathbb{S}), \lambda \geq 1, \tau \in[0,1]$, and $j \in\left\{1, \ldots, 2^{p+1}\right\}$. The arguments at the very and of the proof of Theorem 4.3 enable us to conclude the existence of constants $\beta \in(0,1)$ and $\omega_{0} \geq 1$ with

$$
\|(\lambda+B(\tau))[f]\|_{H^{1}} \geq \beta\|f\|_{H^{2}}
$$

for all $f \in H^{2}(\mathbb{S}), \lambda \geq \omega_{0}$, and $\tau \in[0,1]$. The continuity method [5, Proposition I.1.1.1] and the previous observation that $\lambda+B(0) \in \operatorname{Isom}\left(H^{2}(\mathbb{S}), H^{1}(\mathbb{S})\right)$ for $\lambda>0$ yield the desired conclusion.

We are now in a position to derive the desired generator property (5.2).

Theorem 5.4. Given $f_{0} \in \mathcal{O}$, it holds that

$$
-\partial \Phi\left(f_{0}\right) \in \mathcal{H}\left(H^{2}(\mathbb{S}), H^{1}(\mathbb{S})\right) .
$$

Proof. Given $f_{0} \in \mathcal{O}$ and $\tau \in[0,1]$, let $\alpha_{\tau}$ and $\beta_{\tau}$ denote the functions introduced in Theorem 5.2. The Rayleigh-Taylor condition $a_{\mathrm{RT}}>0$ ensures there exists a constant $\eta \in(0,1)$ such that

$$
\eta \leq \alpha_{\tau} \leq \frac{1}{\eta} \quad \text { and } \quad\left|\beta_{\tau}\right| \leq \frac{1}{\eta}
$$

for all $\tau \in[0,1]$. Given $\alpha \in[\eta, 1 / \eta]$ and $|\beta|<1 / \eta$, let $\mathbb{A}_{\alpha, \beta}$ denote the Fourier multiplier

$$
\mathbb{A}_{\alpha, \beta}:=-\alpha\left(-\partial_{x}^{2}\right)^{1 / 2}+\beta \partial_{x} .
$$


It is not difficult to prove there exists $\kappa_{0} \geq 1$ such that the complexification of $\mathbb{A}_{\alpha, \beta}$ (denoted again by $\left.\mathbb{A}_{\alpha, \beta}\right)$ satisfies

$$
\kappa_{0}\left\|\left(\lambda-\mathbb{A}_{\alpha, \beta}\right)[f]\right\|_{H^{1}} \geq|\lambda| \cdot\|f\|_{H^{1}}+\|f\|_{H^{2}}
$$

for all $\alpha \in[\eta, 1 / \eta],|\beta|<1 / \eta, \operatorname{Re} \lambda \geq 1$, and $f \in H^{2}(\mathbb{S})$. Observing that the operators $\mathbb{A}_{j, \tau}$ found in Theorem 5.2 belong to the family $\left\{\mathbb{A}_{\alpha, \beta}: \alpha \in[\eta, 1 / \eta],|\beta|<1 / \eta\right\}$ and that

$$
\lambda-\Psi(0)=\lambda+H\left[a_{\mathrm{RT}} \partial_{x}\right] \in \operatorname{Isom}\left(H^{2}(\mathbb{S}), H^{1}(\mathbb{S})\right)
$$

for all $\lambda \in \mathbb{R}$ which are sufficiently large, cf. Proposition 5.3, the arguments in the proof of Theorem 4.3 together with (5.29) and Theorem 5.2 lead us to the desired claim.

We conclude this section with the proof of Theorem 1.5.

Proof of Theorem 1.5. The proof follows by using the fully nonlinear parabolic theory in [46, Chapter 8], (5.6), and Theorem 5.4. The details of proof are identical to those in the nonperiodic case, cf. [48, Theorem 1.2], and therefore we omit them.

\section{Stability Analysis}

In this section we identify the equilibria of the Muskat problem (1.1) and study their stability properties. The Muskat problem without surface tension We first infer from Remark 3.4 that $f \in H^{2}(\mathbb{S})$ is a stationary solution to (1.1) (with $\sigma=0$ ) if and only if $f$ is constant also with respect to $x$. Besides, as pointed out in Sect. 5, if $f$ is a solution to (1.1) as found in Theorem 1.5, then $\Phi(f(t)) \in \widehat{H}^{1}(\mathbb{S})$ for all $t$ in the existence interval of $f$, hence the mean integral of the initial datum is preserved by the flow. Recalling also the invariance property (1.2), we shall only address the stability issue for the 0 equilibrium under perturbed initial data with zero integral mean. Hence, we are led to consider the evolution problem

$$
\dot{f}(t)=\Phi(f(t)), \quad t \geq 0, \quad f(0)=f_{0},
$$

where

$$
\Phi \in C^{\omega}\left(\widehat{H}^{2}(\mathbb{S}), \widehat{H}^{1}(\mathbb{S})\right)
$$

is the restriction of the operator defined in (5.5). Recalling (5.8), it follows from the relations $\bar{\omega}(0)=0$, $\mathbb{A}(0)=0$, and $\mathbb{B}(0)=H$, that

$$
\partial \Phi(0)=-c_{\Theta} H \circ \partial_{x}=-c_{\Theta}\left(-\partial_{x}^{2}\right)^{1 / 2} \in \mathcal{L}\left(\widehat{H}^{2}(\mathbb{S}), \widehat{H}^{1}(\mathbb{S})\right),
$$

which identifies the spectrum $\sigma(\partial \Phi(0))$ as being the set

$$
\sigma(\partial \Phi(0))=\left\{-c_{\Theta}|k|: k \in \mathbb{Z} \backslash\{0\}\right\} .
$$

Moreover, it is easy to verify that this Fourier multiplier is the generator of a strongly continuous and analytic semigroup in $\mathcal{L}\left(\widehat{H}^{1}(\mathbb{S})\right)$. This enable us to use the fully nonlinear principle of linearized stability, cf. [46, Theorem 9.1.1], and prove in this way the exponential stability of the zero solution.

Proof of Theorem 1.6. The claim follows from (6.2), the property $-\partial \Phi(0) \in \mathcal{H}\left(\widehat{H}^{2}(\mathbb{S}), \widehat{H}^{1}(\mathbb{S})\right)$, and the fact that $\operatorname{Re} \lambda \leq-c_{\Theta}$ for all $\lambda \in \sigma(\partial \Phi(0))$ via [46, Theorem 9.1.1].

The Muskat Problem with Surface Tension For $\sigma>0$ the stability analysis is more intricate. Before presenting the complete picture of the equilibria we notice that also in this case the mean value of the initial data is preserved by the flow. This aspect and the invariance property (1.2) enable us to restrict our stability analysis to the setting of solutions with zero integral mean.

In view of Remark 3.4, a function $f \in \widehat{H}^{3}(\mathbb{S})$ is a stationary solution to (1.1) if and only if it solves the capillarity equation

$$
\frac{f^{\prime \prime}}{\left(1+f^{\prime 2}\right)^{3 / 2}}+\lambda f=0 \quad \text { where } \lambda:=-\frac{\Theta}{\sigma}
$$



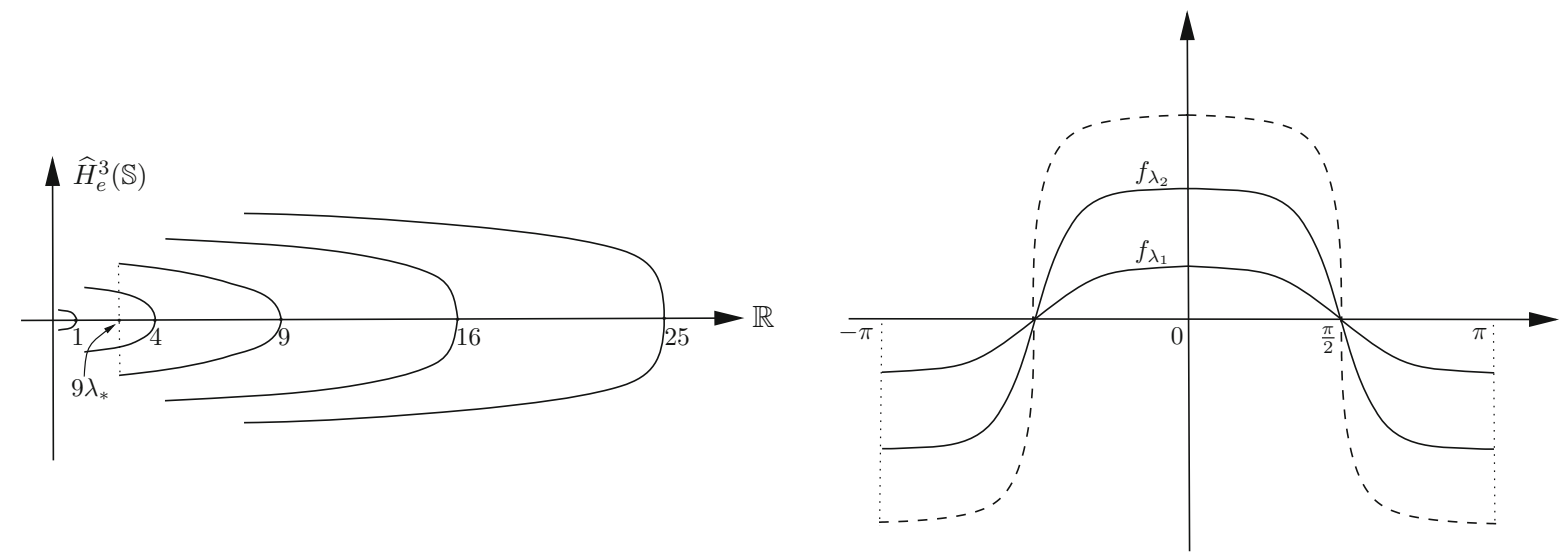

FIG. 1. The subcritical global bifurcation branches of (6.3) found in Theorem 6.1 (left) and the behavior of the finger-shaped solutions along the first bifurcation branch (right) $\left(\lambda_{*}<\lambda_{2}<\lambda_{1}<1\right)$. The dashed curve is the graph of the function $\lim _{\lambda \searrow \lambda_{*}} f_{\lambda}$ and it has unbounded slope at $x=\pi / 2$ and height $\sqrt{2 / \lambda_{*}}$

This equation has been discussed in detail in [28]. If $\lambda \leq 0$, the Eq. (6.3) has by the elliptic maximum principle a unique solution in $\widehat{H}^{3}(\mathbb{S})$, the trivial equilibrium $f=0$. However, if $\lambda>0$, there may exist also finger-shaped solutions to (6.3), see Fig. 1, which are all symmetric with respect to the horizontal lines through the extrema but also with respect to the points where they intersect the $x$-axis. In particular, each equilibrium in $\widehat{H}^{3}(\mathbb{S})$ is the horizontal translation of an even equilibrium. We now view $\lambda>0$ as a bifurcation parameter in the Eq. (6.3) and we shall refer to $(\lambda, f)$ as being the solution to (6.3). The following theorem provides a complete description of the set of even equilibria to the Muskat problem with surface tension (and in virtue of (1.2) also of the set of all equilibria).

Theorem 6.1. Let

$$
\lambda_{*}:=\frac{1}{2 \pi^{2}} B^{2}\left(\frac{3}{4}, \frac{1}{2}\right),
$$

where $B$ is the beta function. The even solutions to (6.3) are organized as follows.

(a) If $\lambda \leq \lambda_{*},{ }^{5}$ then (6.3) has only the trivial solution.

(b) Let $\lambda>\lambda_{*}$.

(i) The Eq. (6.3) has even solutions of minimal period $2 \pi$ if and only if $\lambda_{*}<\lambda<1$. More precisely, for each $\lambda \in\left(\lambda_{*}, 1\right)$, (6.3) has exactly two even solutions $\left(\lambda, \pm f_{\lambda}\right)$ of minimal period $2 \pi$. These solutions are real-analytic, $\left|f_{\lambda_{1}}\right| \leq\left|f_{\lambda_{2}}\right|$ for $\lambda_{2}<\lambda_{1}$, $\left\|f_{\lambda}\right\|_{\infty} \rightarrow 0$ for $\lambda \nearrow 1$, and

$$
\left\|f_{\lambda}\right\|_{\infty}=\left|f_{\lambda}(0)\right| \nearrow \sqrt{2 / \lambda_{*}}, \quad\left\|f_{\lambda}^{\prime}\right\|_{\infty}=\left|f_{\lambda}^{\prime}(\pi / 2)\right| \nearrow \infty \quad \text { for } \lambda \searrow \lambda_{*} .
$$

(ii) The Eq. (6.3) has even solutions of minimal period $2 \pi / \ell, 2 \leq \ell \in \mathbb{N}$, if and only if $\ell^{2} \lambda_{*}<$ $\lambda<\ell^{2}$. More precisely, for each $\lambda \in\left(\ell^{2} \lambda_{*}, \ell^{2}\right),(6.3)$ has exactly two even solutions $\left(\lambda, \pm f_{\lambda}\right)$ of minimal period $2 \pi / \ell$ and

$$
f_{\lambda}=\ell^{-1} f_{\lambda \ell^{-2}}(\ell \cdot)
$$

where $f_{\lambda \ell^{-2}}(\ell \cdot)$ is the function identified at $(i i)$.

(c) If we consider (6.3) as an abstract bifurcation problem in $\mathbb{R} \times \widehat{H}_{e}^{3}(\mathbb{S})$, where

$$
\widehat{H}_{e}^{3}(\mathbb{S}):=\left\{f \in H_{e}^{3}(\mathbb{S}): f \text { is even }\right\},
$$

\footnotetext{
${ }^{5} \mathrm{~A}$ rough estimate for $\lambda_{*}$ is $\lambda_{*} \approx 0.3$.
} 
then the global bifurcation curve arising from $\left(\ell^{2}, 0\right), 1 \leq \ell \in \mathbb{N}$, and described at $(b)$, admits in a neighborhood of $\left(\ell^{2}, 0\right)$ a real-analytic parametrization

$$
\left(\lambda_{\ell}, f_{\ell}\right):\left(-\varepsilon_{\ell}, \varepsilon_{\ell}\right) \rightarrow(0, \infty) \times \widehat{H}_{e}^{3}(\mathbb{S})
$$

such that

$$
\left\{\begin{array}{l}
\lambda_{\ell}(s)=\ell^{2}-\frac{3 \ell^{4}}{8} s^{2}+O\left(s^{4}\right) \quad \text { in } \mathbb{R}, \\
f_{\ell}(s)=s \cos (\ell x)+O\left(s^{2}\right)
\end{array} \quad \text { in } \widehat{H}_{e}^{3}(\mathbb{S}) \quad \text { for } s \rightarrow 0 .\right.
$$

Proof. The claims $(a)$ and $(b)$ are established in [28]. The last claim follows by applying the theorem on bifurcations from simple eigenvalues due to Crandall and Rabinowitz, cf. [19]. The details are similar to those in the proof of [31, Theorem 6.1].

With respect to Theorem 6.1 we add the following remark.

Remark 6.2. (i) Because $\lambda_{*} \approx 0.3$, for certain $\lambda \in\left(\ell^{2} \lambda_{*}, \ell^{2}\right)$ with $\ell \geq 2$ there exist nontrivial solutions to (6.3) with minimal period different than $2 \pi / \ell$, see Fig. 1.

(ii) As pointed out in [28], these finger-shaped equilibria are in correspondence to certain solutions to the mathematical pendulum equation

$$
\theta^{\prime \prime}+\lambda \sin \theta=0 .
$$

(iii) The global bifurcation curves may be continued beyond $\lambda_{*} \ell^{2}$, but outside the setting of interfaces parametrized as graphs.

(iv) Because $\lambda_{\ell}^{\prime}(0)=0>\lambda_{\ell}^{\prime \prime}(0)$, we may assume that $s \lambda_{\ell}^{\prime}(s)<0$ for all $s \in\left(-\varepsilon_{\ell}, \varepsilon_{\ell}\right) \backslash\{0\}$. This aspect is of relevance when studying the stability properties of the finger-shaped equilibria identified above.

In order to address the stability properties of the equilibria to (1.1), we first reformulate the problem by incorporating $\lambda$ as a parameter. To this end we define $\Phi: \mathbb{R} \times \widehat{H}^{2}(\mathbb{S}) \rightarrow \mathcal{L}\left(\widehat{H}^{3}(\mathbb{S}), \widehat{L}_{2}(\mathbb{S})\right)$ according to

$$
\Phi(\lambda, f)[h]:=\sigma b_{\mu} \mathbb{B}(f)\left[\left(1+a_{\mu} \mathbb{A}(f)\right)^{-1}\left[\frac{h^{\prime \prime \prime}}{\left(1+f^{\prime 2}\right)^{3 / 2}}-3 \frac{f^{\prime} f^{\prime \prime} h^{\prime \prime}}{\left(1+f^{\prime 2}\right)^{3 / 2}}+\lambda h^{\prime}\right],\right.
$$

where $b_{\mu}$ is the constant introduced in (4.4). Then, it follows from the analysis in Sect. 4 that $\Phi \in$ $C^{\omega}\left(\mathbb{R} \times \widehat{H}^{2}(\mathbb{S}), \mathcal{L}\left(\widehat{H}^{3}(\mathbb{S}), \widehat{L}_{2}(\mathbb{S})\right)\right)$, and the problem (1.1) is equivalent, for solutions with zero integral mean, to the quasilinear evolution problem

$$
\dot{f}(t)=\Phi(\lambda, f(t))[f(t)], \quad t>0, \quad f(0)=f_{0} .
$$

It is not difficult to see that the linearization $\Phi(\lambda, 0) \in \mathcal{L}\left(\widehat{H}^{3}(\mathbb{S}), \widehat{L}_{2}(\mathbb{S})\right)$ is a Fourier multiplier with spectrum $\sigma(\Phi(\lambda, 0))$ that consists only of the eigenvalues $\left\{-\sigma b_{\mu}\left(|k|^{3}-\lambda|k|\right): k \in \mathbb{Z} \backslash\{0\}\right\}$. Moreover, $\Phi(\lambda, 0)$ generates a strongly continuous and analytic semigroup in $\mathcal{L}\left(\widehat{L}_{2}(\mathbb{S})\right)$ for all $\lambda \in \mathbb{R}$. We are now in a position to prove Theorem 1.3 where we exploit the quasilinear principle of linearized stability in [50, Theorem 1.3].

Proof of Theorem 1.3. We first address the stability of the zero solution $f=0$ to (1.1). Assume first that $\lambda<1$. In this case all eigenvalues of $\Phi(\lambda, 0)$ are negative, more precisely $\operatorname{Re} z \leq-\sigma b_{\mu}(1-\lambda)<0$ for all $z \in \sigma(\Phi(\lambda, 0))$. The quasilinear principle of linearized stability [50, Theorem 1.3] applied to (6.5) yields the first claim of Theorem 1.3.

In the second case when $\lambda>1$, the intersection $\sigma\left(\partial_{f} \Phi(\lambda, 0)\right) \cap[\operatorname{Re} \lambda>0]$ consists of a finite number of positive eigenvalues and we may apply the instability result in [50, Theorem 1.4] to derive the assertion (ii) in Theorem 1.3.

In the remaining part we discuss the stability properties of small finger-shaped solutions. To this end we denote by $\mathbb{A}_{\ell}(s)$ the linearized operator

$$
\mathbb{A}_{\ell}(s):=\Phi\left(\lambda_{\ell}(s), f_{\ell}(s)\right)+\left(\partial_{f} \Phi\left(\lambda_{\ell}(s), f_{\ell}(s)\right)[\cdot]\right)\left[f_{\ell}(s)\right] \in \mathcal{L}\left(\widehat{H}^{3}(\mathbb{S}), \widehat{L}_{2}(\mathbb{S})\right),
$$


where $\partial_{f} \Phi \in \mathcal{L}\left(\widehat{H}^{2}(\mathbb{S}), \mathcal{L}\left(\widehat{H}^{3}(\mathbb{S}), \widehat{L}_{2}(\mathbb{S})\right)\right)$ is the Fréchet derivative of the mapping $\Phi$ with respect to the variable $f$. We point out that $\mathbb{A}_{\ell}(0)=\Phi\left(\ell^{2}, 0\right)$.

Let us first note that for $\ell \geq 2$ the spectrum $\sigma\left(\mathbb{A}_{\ell}(0)\right)$ contains a finite number of positive eigenvalues (this number increases with $\ell$ ). Since a set consisting of finitely many eigenvalues of $\mathbb{A}_{\ell}(s)$ changes continuously with $s \in\left(-\varepsilon_{\ell}, \varepsilon_{\ell}\right)$, cf. [44, Chapter IV], we infer from [5, Theorem I.1.3.1 (i)] that $-\mathbb{A}(s) \in$ $\mathcal{H}\left(\widehat{H}^{3}(\mathbb{S}), \widehat{L}_{2}(\mathbb{S})\right)$ and that $\sigma(\mathbb{A}(s))$ contains only finitely many eigenvalues with positive real part if $\varepsilon_{\ell}$ is sufficiently small. Thus, we may appeal to [50, Theorem 1.4] to conclude that if $\lambda=\lambda_{\ell}(s), 0<|s|<\varepsilon_{\ell}$, $\ell \geq 2$, then $f_{\ell}(s)$ is an unstable equilibrium to $(1.1)$.

The situation when $\ell=1$ is special, because $\sigma\left(\mathbb{A}_{1}(s)\right)$ has for $s=0$, except for the eigenvalue 0 , only negative eigenvalues. We show below that when letting $s$ vary in $\left(-\varepsilon_{1}, \varepsilon_{1}\right)$ the operator $\mathbb{A}_{1}(s), 0<|s|<1$, has a positive eigenvalue $z(s)$ which corresponds to the zero eigenvalue of $\mathbb{A}_{1}(0)$. To this end we associate to a periodic function $h$ the function $\check{h}$ defined by

$$
\breve{h}(x):=h(-x), \quad x \in \mathbb{R} .
$$

Observing that $(\mathbb{B}(f)[\bar{\omega}])^{\llcorner}=-\mathbb{B}(\check{f})[\check{\omega}]$ and $(\mathbb{A}(f)[\bar{\omega}])^{\llcorner}=\mathbb{A}(\check{f})[\check{\omega}], f \in \widehat{H}^{2}(\mathbb{S}), \bar{\omega} \in \widehat{L}_{2}(\mathbb{S})$, and that

$$
\bar{\omega}(\check{f})[\check{h}]=-(\bar{\omega}(f)[h])^{\nu}, \quad f \in \widehat{H}^{2}(\mathbb{S}), h \in \widehat{H}^{3}(\mathbb{S}),
$$

cf. Proposition 4.1, it follows that the operator $\Phi$ introduced in (6.4) satisfies

$$
(\Phi(\lambda, f)[h])^{\llcorner}=\Phi(\lambda, \check{f})[\check{h}] \quad \text { for } \lambda \in \mathbb{R}, f \in \widehat{H}^{2}(\mathbb{S}), h \in \widehat{H}^{3}(\mathbb{S}) .
$$

Hence, letting $\widehat{L}_{2, e}(\mathbb{S}):=\left\{f \in \widehat{L}_{2}(\mathbb{S})\right.$ : fis even $\}$ and $\widehat{H}_{e}^{r}(\mathbb{S}):=\widehat{H}^{r}(\mathbb{S}) \cap L_{2, e}(\mathbb{S}), r \geq 0$, it follows that $\Phi \in C^{\omega}\left(\mathbb{R} \times \widehat{H}_{e}^{2}(\mathbb{S}), \mathcal{L}\left(\widehat{H}_{e}^{3}(\mathbb{S}), \widehat{L}_{2, e}(\mathbb{S})\right)\right)$, the linearization $\mathbb{A}_{1}(0) \in \mathcal{L}\left(\widehat{H}_{e}^{3}(\mathbb{S}), \widehat{L}_{2, e}(\mathbb{S})\right)$ being the Fourier multiplier

$$
\mathbb{A}_{1}(0) \sum_{k=1}^{\infty} a_{k} \cos (k x)=-\sigma b_{\mu} \sum_{k=1}^{\infty}\left(k^{3}-\lambda k\right) a_{k} \cos (k x) .
$$

Let $\Psi: \mathbb{R} \times \widehat{H}_{e}^{3}(\mathbb{S}) \rightarrow \widehat{L}_{2, e}(\mathbb{S})$ be the real-analytic mapping defined by $\Psi(\lambda, f):=\Phi(\lambda, f)[f]$. Noticing that $\partial_{f} \Psi\left(\lambda_{1}(s), f_{1}(s)\right)=\mathbb{A}_{1}(s)$, it follows that 0 is a simple eigenvalue of $\partial_{f} \Psi(1,0)$ and $\operatorname{Ker} \partial_{f} \Psi(1,0)=$ $\operatorname{span}\{\cos (x)\}$. Since additionally $\partial_{\lambda f} \Psi(1,0)[\cos (x)]=\sigma b_{\mu} \cos (x) \notin \operatorname{Im} \partial_{f} \Psi(1,0)$, the principle of exchange of stability, cf. [20, Theorem 1.16], together with Remark 6.2 (iv) implies that the zero eigenvalue of $\partial_{f} \Psi(1,0)$ perturbs along the bifurcation curve through $\left(\lambda_{1}, f_{1}\right)$ into a positive eigenvalue $z(s)$ of $\mathbb{A}_{1}(s)$, $0<|s|<\varepsilon_{1}$, and moreover

$$
\lim _{s \rightarrow 0} \frac{-s \lambda_{1}^{\prime}(s)}{z(s)}=\frac{1}{\sigma b_{\mu}} .
$$

Hence, if $\varepsilon_{1}$ is sufficiently small, the operator $\mathbb{A}_{1}(s), 0<|s|<\varepsilon_{1}$, has a positive eigenvalue $z(s)$. Moreover, $\mathbb{A}_{1}(s)$ has at most two eigenvalues with positive real part. [50, Theorem 1.4] yields now that if $\lambda=\lambda_{1}(s)$, $0<|s|<\varepsilon_{1}$, then $f_{1}(s)$ is an unstable equilibrium.

Acknowledgements. Open Access funding provided by Projekt DEAL.

\section{Compliance with Ethical Standards}

Conflicts of interest The author declares that he has no conflict of interest.

Open Access. This article is licensed under a Creative Commons Attribution 4.0 International License, which permits use, sharing, adaptation, distribution and reproduction in any medium or format, as long as you give appropriate credit to the original author(s) and the source, provide a link to the Creative Commons licence, and indicate if changes were made. The images or other third party material in this article are included in the article's Creative Commons licence, unless indicated otherwise in a credit line to the material. If material is not included in the article's Creative Commons licence and your 
intended use is not permitted by statutory regulation or exceeds the permitted use, you will need to obtain permission directly from the copyright holder. To view a copy of this licence, visit http://creativecommons.org/licenses/by/4.0/.

Publisher's Note Springer Nature remains neutral with regard to jurisdictional claims in published maps and institutional affiliations.

\section{Appendix A. Some Technical Results}

In Lemma A.1 we establish the boundedness of a family of multilinear singular integral operators in certain settings that are motivated by the analysis in the previous sections. The nonperiodic counterparts of the estimates derived below have been obtained previously in $[48,49] .{ }^{6}$

Lemma A.1. (i) Given $m, n \in \mathbb{N}$ and Lipschitz functions $a_{1}, \ldots, a_{m}, b_{1}, \ldots, b_{n}: \mathbb{R} \rightarrow \mathbb{R}$, the singular integral operator $C_{n, m}\left(a_{1}, \ldots, a_{m}\right)\left[b_{1}, \ldots, b_{n}, \cdot\right]$ defined by

$$
C_{n, m}\left(a_{1}, \ldots, a_{m}\right)\left[b_{1}, \ldots, b_{n}, \bar{\omega}\right](x):=\mathrm{PV} \int_{-\pi}^{\pi} \frac{\bar{\omega}(x-s)}{s} \frac{\prod_{i=1}^{n}\left(\delta_{[x, s]} b_{i} / s\right)}{\prod_{i=1}^{m}\left[1+\left(\delta_{[x, s]} a_{i} / s\right)^{2}\right]} \mathrm{d} s
$$

satisfies $\left\|C_{n, m}\left(a_{1}, \ldots, a_{m}\right)\left[b_{1}, \ldots, b_{n}, \cdot\right]\right\|_{\mathcal{L}\left(L_{2}(\mathbb{S}), L_{2}((-\pi, \pi))\right)} \leq C \prod_{i=1}^{n}\left\|b_{i}^{\prime}\right\|_{\infty}$, with a constant $C$ that depends only on $n, m$ and $\max _{i=1, \ldots, m}\left\|a_{i}^{\prime}\right\|_{\infty}$.

In particular, $C_{n, m} \in \mathrm{C}^{1-}\left(\left(W_{\infty}^{1}(\mathbb{S})\right)^{m}, \mathcal{L}_{n+1}\left(\left(W_{\infty}^{1}(\mathbb{S})\right)^{n} \times L_{2}(\mathbb{S}), L_{2}(\mathbb{S})\right)\right)$.

(ii) Let $m \in \mathbb{N}, 1 \leq n \in \mathbb{N}, r \in(3 / 2,2)$, and $\tau \in(5 / 2-r, 1)$. Then:

(ii1) Given $a_{1}, \ldots, a_{m} \in H^{r}(\mathbb{S})$ and $b_{1}, \ldots, b_{n}, \bar{\omega} \in \mathrm{C}^{\infty}(\mathbb{S})$, there exists a constant $C$ that depends only on $n, m, r, \tau$, and $\max _{i=1, \ldots, m}\left\|a_{i}\right\|_{H^{r}(\mathbb{S})}$ such that

$$
\left\|C_{n, m}\left(a_{1}, \ldots, a_{m}\right)\left[b_{1}, \ldots, b_{n}, \bar{\omega}\right]\right\|_{L_{2}(\mathbb{S})} \leq C\|\bar{\omega}\|_{H^{\tau}(\mathbb{S})}\left\|b_{1}\right\|_{H^{1}(\mathbb{S})} \prod_{i=2}^{n}\left\|b_{i}\right\|_{H^{r}(\mathbb{S})}
$$

and

$$
\begin{aligned}
& \left\|C_{n, m}\left(a_{1}, \ldots, a_{m}\right)\left[b_{1}, \ldots, b_{n}, \bar{\omega}\right]-C_{n-1, m}\left(a_{1}, \ldots, a_{m}\right)\left[b_{2}, \ldots, b_{n}, b_{1}^{\prime} \bar{\omega}\right]\right\|_{L_{2}(\mathbb{S})} \\
& \quad \leq C\left\|b_{1}\right\|_{H^{\tau}(\mathbb{S})}\|\bar{\omega}\|_{H^{1}(\mathbb{S})} \prod_{i=2}^{n}\left\|b_{i}\right\|_{H^{r}(\mathbb{S})} .
\end{aligned}
$$

In particular, $C_{n, m}\left(a_{1}, \ldots, a_{m}\right)$ has an extension in

$$
\mathcal{L}_{n+1}\left(H^{1}(\mathbb{S}) \times\left(H^{r}(\mathbb{S})\right)^{n-1} \times H^{\tau}(\mathbb{S}), L_{2}(\mathbb{S})\right)
$$

(ii2) $C_{n, m} \in \mathrm{C}^{1-}\left(\left(H^{r}(\mathbb{S})\right)^{m}, \mathcal{L}_{n+1}\left(H^{1}(\mathbb{S}) \times\left(H^{r}(\mathbb{S})\right)^{n-1} \times H^{\tau}(\mathbb{S}), L_{2}(\mathbb{S})\right)\right)$.

(iii) Let $m, n \in \mathbb{N}, r \in(3 / 2,2)$, and $\tau \in(1 / 2,1)$. Then:

(iii1) Given $a_{1}, \ldots, a_{m} \in H^{r}(\mathbb{S})$ and $b_{1}, \ldots, b_{n}, \bar{\omega} \in \mathrm{C}^{\infty}(\mathbb{S})$, there exists a constant $C$ that depends only on $n, m, r, \tau$, and $\max _{i=1, \ldots, m}\left\|a_{i}\right\|_{H^{r}(\mathbb{S})}$ such that

$$
\left\|C_{n, m}\left(a_{1}, \ldots, a_{m}\right)\left[b_{1}, \ldots, b_{n}, \bar{\omega}\right]\right\|_{\infty} \leq C\|\bar{\omega}\|_{H^{\tau}(\mathbb{S})} \prod_{i=1}^{n}\left\|b_{i}\right\|_{H^{r}(\mathbb{S})} .
$$

In particular, $C_{n, m}\left(a_{1}, \ldots, a_{m}\right)$ has an extension in $\mathcal{L}_{n+1}\left(\left(H^{r}(\mathbb{S})\right)^{n} \times H^{\tau}(\mathbb{S}), L_{\infty}(\mathbb{S})\right)$.

(iii2) $C_{n, m} \in \mathrm{C}^{1-}\left(\left(H^{r}(\mathbb{S})\right)^{m}, \mathcal{L}_{n+1}\left(\left(H^{r}(\mathbb{S})\right)^{n} \times H^{\tau}(\mathbb{S}), L_{\infty}(\mathbb{S})\right)\right)$.

${ }^{6}$ In $[48,49]$ the operators

$$
B_{n, m}\left(a_{1}, \ldots, a_{m}\right)\left[b_{1}, \ldots, b_{n}, \bar{\omega}\right](x):=\mathrm{PV} \int_{\mathbb{R}} \frac{\bar{\omega}(x-s)}{s} \frac{\prod_{i=1}^{n}\left(\delta_{[x, s]} b_{i} / s\right)}{\prod_{i=1}^{m}\left[1+\left(\delta_{[x, s]} a_{i} / s\right)^{2}\right]} \mathrm{d} s
$$

are considered. The functions $a_{1}, \ldots, a_{m}, b_{1}, \ldots, b_{n}: \mathbb{R} \rightarrow \mathbb{R}$ are Lipschitz functions and $\bar{\omega} \in L_{2}(\mathbb{R})$. It is shown in [48,49] that these operators extend to bounded multilinear operators on certain products of Sobolev spaces on $\mathbb{R}$. 
Proof. We first address $(i)$. To this end we fix $\varphi \in \mathrm{C}_{0}^{\infty}(\mathbb{R},[0,1])$ with $\varphi=1$ for $|x| \leq 2 \pi$ and $\varphi=0$ for $|x| \geq 4 \pi$. Then, it is easy to see that

$$
\|\bar{\omega}\|_{L_{2}(\mathbb{S})} \leq\|\bar{\omega} \varphi\|_{L_{2}(\mathbb{R})} \leq 4\|\bar{\omega}\|_{L_{2}(\mathbb{S})} \quad \text { for all } \bar{\omega} \in L_{2}(\mathbb{S}) .
$$

For $|x|<\pi$ we have

$$
\begin{aligned}
C_{n, m}\left(a_{1}, \ldots, a_{m}\right)\left[b_{1}, \ldots, b_{n}, \bar{\omega}\right](x)= & B_{n, m}\left(a_{1}, \ldots, a_{m}\right)\left[b_{1}, \ldots, b_{n}, \varphi \bar{\omega}\right](x) \\
& -\int_{\pi<|s|<5 \pi} \frac{(\varphi \bar{\omega})(x-s)}{s} \frac{\prod_{i=1}^{n}\left(\delta_{[x, s]} b_{i} / s\right)}{\prod_{i=1}^{m}\left[1+\left(\delta_{[x, s]} a_{i} / s\right)^{2}\right]} \mathrm{d} s,
\end{aligned}
$$

and it follows from [48, Lemma 3.1] and (A.4) that

$$
\left\|B_{n, m}\left(a_{1}, \ldots, a_{m}\right)\left[b_{1}, \ldots, b_{n}, \varphi \bar{\omega}\right]\right\|_{L_{2}((-\pi, \pi))} \leq C\|\varphi \bar{\omega}\|_{L_{2}(\mathbb{R})} \prod_{i=1}^{n}\left\|b_{i}^{\prime}\right\|_{\infty} \leq C\|\bar{\omega}\|_{L_{2}(\mathbb{S})} \prod_{i=1}^{n}\left\|b_{i}^{\prime}\right\|_{\infty} .
$$

Moreover, it holds that

$$
\left\|\int_{\pi<|s|<5 \pi} \frac{(\varphi \bar{\omega})(\cdot-s)}{s} \frac{\prod_{i=1}^{n}\left(\delta_{[\cdot, s]} b_{i} / s\right)}{\prod_{i=1}^{m}\left[1+\left(\delta_{[\cdot, s]} a_{i} / s\right)^{2}\right]} \mathrm{d} s\right\|_{\infty} \leq C\|\bar{\omega}\|_{L_{2}(\mathbb{S})} \prod_{i=1}^{n}\left\|b_{i}^{\prime}\right\|_{\infty} .
$$

Herewith we established the estimate stated at $(i)$. If $a_{1}, \ldots, a_{m}, b_{1}, \ldots, b_{n}$ are $2 \pi$-periodic, then so is also the function $C_{n, m}\left(a_{1}, \ldots, a_{m}\right)\left[b_{1}, \ldots, b_{n}, \bar{\omega}\right]$, and the local Lipschitz continuity property of $C_{n, m}$ follows directly from the estimate.

In order to prove $(i i)$ we start by noticing that for $h \in \mathrm{C}^{\infty}(\mathbb{S})$ it holds that

$$
\frac{\partial}{\partial s}\left(\frac{\delta_{[x, s]} h}{s}\right)=\frac{h^{\prime}(x-s)}{s}-\frac{\delta_{[x, s]} h}{s^{2}}=-\frac{\delta_{[x, s]} h-s h^{\prime}(x-s)}{s^{2}} \quad \text { for } x \in \mathbb{R}, s \neq 0 .
$$

Using this relation we get

$$
\begin{aligned}
C_{n, m}\left(a_{1}, \ldots, a_{m}\right)\left[b_{1}, \ldots, b_{n}, \bar{\omega}\right](x)= & \mathrm{PV} \int_{-\pi}^{\pi} \frac{\delta_{[x, s]} b_{1}}{s^{2}} \frac{\prod_{i=2}^{n}\left(\delta_{[x, s]} b_{i} / s\right)}{\prod_{i=1}^{m}\left[1+\left(\delta_{[x, s]} a_{i} / s\right)^{2}\right]} \bar{\omega}(x-s) \mathrm{d} s \\
= & C_{n-1, m}\left(a_{1}, \ldots, a_{m}\right)\left[b_{2}, \ldots, b_{n}, b_{1}^{\prime} \bar{\omega}\right](x) \\
& -\mathrm{PV} \int_{-\pi}^{\pi} \frac{\partial}{\partial s}\left(\frac{\delta_{[x, s]} b_{1}}{s}\right) \frac{\prod_{i=2}^{n}\left(\delta_{[x, s]} b_{i} / s\right)}{\prod_{i=1}^{m}\left[1+\left(\delta_{[x, s]} a_{i} / s\right)^{2}\right]} \bar{\omega}(x-s) \mathrm{d} s,
\end{aligned}
$$

and the estimate established at $(i)$ yields

$$
\left\|C_{n-1, m}\left(a_{1}, \ldots, a_{m}\right)\left[b_{2}, \ldots, b_{n}, b_{1}^{\prime} \bar{\omega}\right]\right\|_{L_{2}(\mathbb{S})} \leq C\|\bar{\omega}\|_{H^{\tau}(\mathbb{S})}\left\|b_{1}\right\|_{H^{1}(\mathbb{S})} \prod_{i=2}^{n}\left\|b_{i}\right\|_{H^{r}(\mathbb{S})} .
$$

We are left with the singular integral term

$$
\begin{aligned}
\mathrm{PV} & \int_{-\pi}^{\pi} \frac{\partial}{\partial s}\left(\frac{\delta_{[x, s]} b_{1}}{s}\right) \frac{\prod_{i=2}^{n}\left(\delta_{[x, s]} b_{i} / s\right)}{\prod_{i=1}^{m}\left[1+\left(\delta_{[x, s]} a_{i} / s\right)^{2}\right]} \bar{\omega}(x-s) \mathrm{d} s \\
= & \left(1-(-1)^{n}\right) \frac{\prod_{i=1}^{n}\left(\delta_{[x, \pi]} b_{i} / \pi\right)}{\prod_{i=1}^{m}\left[1+\left(\delta_{[x, \pi]} a_{i} / \pi\right)^{2}\right]} \bar{\omega}(x-\pi) \\
& +\left(b_{1} C_{n-1, m}\left(a_{1}, \ldots, a_{m}\right)\left[b_{2}, \ldots, b_{n}, \bar{\omega}^{\prime}\right](x)-C_{n-1, m}\left(a_{1}, \ldots, a_{m}\right)\left[b_{2}, \ldots, b_{n}, b_{1} \bar{\omega}^{\prime}\right]\right)(x) \\
& +\sum_{j=2}^{n} \int_{-\pi}^{\pi} K_{1, j}(x, s) \bar{\omega}(x-s) \mathrm{d} s-2 \sum_{j=1}^{m} \int_{-\pi}^{\pi} K_{2, j}(x, s) \bar{\omega}(x-s) \mathrm{d} s,
\end{aligned}
$$


where

$$
\begin{aligned}
K_{1, j}(x, s) & :=\frac{\prod_{i=1, i \neq j}^{n}\left(\delta_{[x, s]} b_{i} / s\right)}{\prod_{i=1}^{m}\left[1+\left(\delta_{[x, s]} a_{i} / s\right)^{2}\right]} \frac{\delta_{[x, s]} b_{j}-s b_{j}^{\prime}(x-s)}{s^{2}} \\
K_{2, j}(x, s) & :=\frac{\prod_{i=1}^{n}\left(\delta_{[x, s]} b_{i} / s\right)}{\prod_{i=1}^{m}\left[1+\left(\delta_{[x, s]} a_{i} / s\right)^{2}\right]} \frac{\delta_{[x, s]} a_{j} / s}{1+\left(\delta_{[x, s]} a_{j} / s\right)^{2}} \frac{\delta_{[x, s]} a_{j}-s a_{j}^{\prime}(x-s)}{s^{2}}
\end{aligned}
$$

for $x \in \mathbb{R}$ and $s \neq 0$. The relation (A.6) is obtained by using integration by parts. We next estimate the terms on the right hand side of (A.6) separately. Firstly, it is easy to see that

$$
\left\|\frac{\prod_{i=1}^{n}\left(\delta_{[\cdot, \pi]} b_{i} / \pi\right)}{\prod_{i=1}^{m}\left[1+\left(\delta_{[\cdot, \pi]} a_{i} / \pi\right)^{2}\right]} \bar{\omega}(\cdot-\pi)\right\|_{L_{2}(\mathbb{S})} \leq C\|\bar{\omega}\|_{L_{2}(\mathbb{S})}\left\|b_{1}\right\|_{\infty} \prod_{i=2}^{n}\left\|b_{i}\right\|_{H^{r}(\mathbb{S})} .
$$

Secondly, concerning the last two terms in (A.6), we may adapt the arguments from the nonperiodic case [48, Lemma 3.2], to arrive at

$$
\begin{aligned}
& \left(\int_{-\pi}^{\pi}\left|\int_{-\pi}^{\pi} K_{1, j}(x, s) \bar{\omega}(x-s) \mathrm{d} s\right|^{2} \mathrm{~d} x\right)^{1 / 2} \leq C\|\bar{\omega}\|_{\infty}\left\|b_{1}\right\|_{H^{\tau}(\mathbb{S})} \prod_{i=2}^{n}\left\|b_{i}\right\|_{H^{r}(\mathbb{S})}, \quad 2 \leq j \leq n, \\
& \left(\int_{-\pi}^{\pi}\left|\int_{-\pi}^{\pi} K_{2, j}(x, s) \bar{\omega}(x-s) \mathrm{d} s\right|^{2} \mathrm{~d} x\right)^{1 / 2} \leq C\|\bar{\omega}\|_{\infty}\left\|b_{1}\right\|_{H^{\tau}(\mathbb{S})} \prod_{i=2}^{n}\left\|b_{i}\right\|_{H^{r}(\mathbb{S})}, \quad 1 \leq j \leq m .
\end{aligned}
$$

Indeed, since $H^{\tau}(\mathbb{S}) \hookrightarrow \mathrm{C}^{\tau-1 / 2}(\mathbb{S})$, we obtain after appealing to Minkowski's inequality that ${ }^{7}$

$$
\begin{aligned}
& \left(\int_{-\pi}^{\pi}\left|\int_{-\pi}^{\pi} K_{1, j}(x, s) \bar{\omega}(x-s) \mathrm{d} s\right|^{2} \mathrm{~d} x\right)^{1 / 2} \leq \int_{-\pi}^{\pi}\left(\int_{-\pi}^{\pi}\left|K_{1, j}(x, s) \bar{\omega}(x-s)\right|^{2} \mathrm{~d} x\right)^{1 / 2} \mathrm{~d} s \\
& \quad \leq C\|\bar{\omega}\|_{\infty}\left\|b_{1}\right\|_{H^{\tau}(\mathbb{S})}\left(\prod_{i=2, i \neq j}^{n}\left\|b_{i}^{\prime}\right\|_{\infty}\right) \int_{-\pi}^{\pi} s^{\tau-7 / 2}\left(\int_{-\pi}^{\pi}\left|b_{j}-\tau_{s} b_{j}-s \tau_{s} b_{j}^{\prime}\right|^{2}(x) \mathrm{d} x\right)^{1 / 2} \mathrm{~d} s,
\end{aligned}
$$

where, taking into account that $\left|e^{i x}-1-i x\right| \leq 2|x|^{r}$ for all $x \in \mathbb{R}$, we have

$$
\begin{aligned}
\int_{-\pi}^{\pi}\left|b_{j}-\tau_{s} b_{j}-s \tau_{s} b_{j}^{\prime}\right|^{2}(x) \mathrm{d} x & =\sum_{k \in \mathbb{Z}}\left|\widehat{b}_{j}(k)\right|^{2}\left|e^{i k s}-1-i k s\right|^{2} \leq C|s|^{2 r} \sum_{k \in \mathbb{Z}}\left|\widehat{b}_{j}(k)\right|^{2}\left(1+k^{2}\right)^{r} \\
& =C|s|^{2 r}\left\|b_{j}\right\|_{H^{r}(\mathbb{S})}^{2} .
\end{aligned}
$$

Since $r+\tau-7 / 2>-1$, the estimate (A.8) $)_{1}$ follows immediately (similarly for $(\mathrm{A} .8)_{2}$.)

Thirdly, for the remaining term

$$
T:=b_{1} C_{n-1, m}\left(a_{1}, \ldots, a_{m}\right)\left[b_{2}, \ldots, b_{n}, \bar{\omega}^{\prime}\right]-C_{n-1, m}\left(a_{1}, \ldots, a_{m}\right)\left[b_{2}, \ldots, b_{n}, b_{1} \bar{\omega}^{\prime}\right]
$$

in (A.6) we obtain, in virtue of $(i)$, that

$$
\|T\|_{L_{2}(\mathbb{S})} \leq C\|\bar{\omega}\|_{H^{1}(\mathbb{S})}\left\|b_{1}\right\|_{\infty} \prod_{i=2}^{n}\left\|b_{i}\right\|_{H^{r}(\mathbb{S})},
$$

and (A.2) follows from (A.7), (A.8), and (A.9).

In order to derive (A.1), we use the identity $\partial\left(\delta_{[x, s]} \bar{\omega}\right) / \partial s=\bar{\omega}^{\prime}(x-s)$ and integration by parts to recast $T$ as

$$
T(x)=\left(1-(-1)^{n}\right) \frac{\left(\delta_{[x, \pi]} \bar{\omega}\right) \prod_{i=1}^{n}\left(\delta_{[x, \pi]} b_{i} / \pi\right)}{\prod_{i=1}^{m}\left[1+\left(\delta_{[x, \pi]} a_{i} / \pi\right)^{2}\right]}+\sum_{j=1}^{n} \int_{-\pi}^{\pi} K_{1, j}(x, s) \delta_{[x, s]} \bar{\omega} \mathrm{d} s
$$

\footnotetext{
${ }^{7}$ Recall that $\tau_{s}$ stands for the right translation. Moreover, $\widehat{h}(k), k \in \mathbb{Z}$, is the $k$-th Fourier coefficient of $h \in L_{1}(\mathbb{S})$.
} 


$$
-2 \sum_{j=1}^{m} \int_{-\pi}^{\pi} K_{2, j}(x, s) \delta_{[x, s]} \bar{\omega} \mathrm{d} s
$$

with

$$
\left\|\frac{\left(\delta_{[\cdot, \pi]} \bar{\omega}\right) \prod_{i=1}^{n}\left(\delta_{[\cdot, \pi]} b_{i} / \pi\right)}{\prod_{i=1}^{m}\left[1+\left(\delta_{[\cdot, \pi]} a_{i} / \pi\right)^{2}\right]}\right\|_{L_{2}(\mathbb{S})} \leq C\|\bar{\omega}\|_{L_{2}(\mathbb{S})}\left\|b_{1}\right\|_{\infty} \prod_{i=2}^{n}\left\|b_{i}\right\|_{H^{r}(\mathbb{S})} .
$$

Concerning the integral terms in the last sum in (A.10), the embedding $H^{1}(\mathbb{S}) \hookrightarrow \mathrm{C}^{r-3 / 2}(\mathbb{S})$ together with Minkowski's inequality yields

$$
\begin{aligned}
& \left(\int_{-\pi}^{\pi}\left|\int_{-\pi}^{\pi} K_{2, j}(x, s) \delta_{[x, s]} \bar{\omega} \mathrm{d} s\right|^{2} \mathrm{~d} x\right)^{1 / 2} \leq \int_{-\pi}^{\pi}\left(\int_{-\pi}^{\pi}\left|K_{2, j}(x, s) \delta_{[x, s]} \bar{\omega}\right|^{2} \mathrm{~d} x\right)^{1 / 2} \mathrm{~d} s \\
& \leq C\left\|b_{1}\right\|_{H^{1}(\mathbb{S})}\left(\prod_{i=2, i \neq j}^{n}\left\|b_{i}^{\prime}\right\|_{\infty}\right) \int_{-\pi}^{\pi} s^{r-7 / 2}\left(\int_{-\pi}^{\pi}\left|\bar{\omega}-\tau_{s} \bar{\omega}\right|^{2}(x) \mathrm{d} x\right)^{1 / 2} \mathrm{~d} s \\
& =C\left\|b_{1}\right\|_{H^{1}(\mathbb{S})}\left(\prod_{i=2, i \neq j}^{n}\left\|b_{i}^{\prime}\right\|_{\infty}\right) \int_{-\pi}^{\pi} s^{r-7 / 2}\left(\sum_{k \in \mathbb{Z}}|\widehat{\bar{\omega}}(k)|^{2}\left|e^{i k s}-1\right|^{2}\right)^{1 / 2} \mathrm{~d} s \\
& \leq C\left\|b_{1}\right\|_{H^{1}(\mathbb{S})}\left(\prod_{i=2, i \neq j}^{n}\left\|b_{i}^{\prime}\right\|_{\infty}\right) \int_{-\pi}^{\pi} s^{r+\tau-7 / 2}\left(\sum_{k \in \mathbb{Z}}|\widehat{\omega}(k)|^{2}\left(1+k^{2}\right)^{\tau}\right)^{1 / 2} \mathrm{~d} s \\
& =C\|\bar{\omega}\|_{H^{\tau}(\mathbb{S})}\left\|b_{1}\right\|_{H^{1}(\mathbb{S})} \prod_{i=2}^{n}\left\|b_{i}\right\|_{H^{r}(\mathbb{S})}, \quad 1 \leq j \leq m,
\end{aligned}
$$

where we have used the relation $\left|e^{i x}-1\right| \leq C|x|^{\tau}, x \in \mathbb{R}$, when deriving the fourth line.

Similarly, we find for $2 \leq j \leq n$ that

$$
\left(\int_{-\pi}^{\pi}\left|\int_{-\pi}^{\pi} K_{1, j}(x, s) \delta_{[x, s]} \bar{\omega} \mathrm{d} s\right|^{2} \mathrm{~d} x\right)^{1 / 2} \leq C\|\bar{\omega}\|_{H^{\tau}(\mathbb{S})}\left\|b_{1}\right\|_{H^{1}(\mathbb{S})} \prod_{i=2}^{n}\left\|b_{i}\right\|_{H^{r}(\mathbb{S})} .
$$

In the special case when $j=1$, we use the procedure which led to (A.12) together with $(i)$ to conclude that

$$
\begin{aligned}
\left(\int_{-\pi}^{\pi}\left|\int_{-\pi}^{\pi} K_{1,1}(x, s) \delta_{[x, s]} \bar{\omega} \mathrm{d} s\right|^{2} \mathrm{~d} x\right)^{1 / 2} \leq & C\|\bar{\omega}\|_{H^{\tau}(\mathbb{S})}\left\|b_{1}\right\|_{H^{1}(\mathbb{S})} \prod_{i=2}^{n}\left\|b_{i}\right\|_{H^{r}(\mathbb{S})} \\
& +\left\|\bar{\omega} C_{n-1, m}\left(a_{1}, \ldots, a_{m}\right)\left[b_{2}, \ldots, b_{n}, b_{1}^{\prime}\right]\right\|_{2} \\
& +\left\|C_{n-1, m}\left(a_{1}, \ldots, a_{m}\right)\left[b_{2}, \ldots, b_{n}, \bar{\omega} b_{1}^{\prime}\right]\right\|_{2} \\
\leq & C\|\bar{\omega}\|_{H^{\tau}(\mathbb{S})}\left\|b_{1}\right\|_{H^{1}(\mathbb{S})} \prod_{i=2}^{n}\left\|b_{i}\right\|_{H^{r}(\mathbb{S})} .
\end{aligned}
$$

The property (A.1) follows now from (A.5), (A.7), (A.8), and (A.11)-(A.14). The extension property left at (ii1) follows from (A.1). The claim (ii2) is a straight forward consequence of (A.1).

With respect to $($ iii) we decompose

$$
C_{n, m}\left(a_{1}, \ldots, a_{m}\right)\left[b_{1}, \ldots, b_{n}, \bar{\omega}\right]=\bar{\omega} A-B,
$$

with

$$
A(x):=\mathrm{PV} \int_{-\pi}^{\pi} \frac{1}{s} \frac{\prod_{i=1}^{n}\left(\delta_{[x, s]} b_{i} / s\right)}{\prod_{i=1}^{m}\left[1+\left(\delta_{[x, s]} a_{i} / s\right)^{2}\right]} \mathrm{d} s \quad \text { and } \quad B(x):=\int_{-\pi}^{\pi} \frac{\left(\delta_{[x, s]} \bar{\omega} / s\right) \prod_{i=1}^{n}\left(\delta_{[x, s]} b_{i} / s\right)}{\prod_{i=1}^{m}\left[1+\left(\delta_{[x, s]} a_{i} / s\right)^{2}\right]} \mathrm{d} s .
$$


Since $\tau>1 / 2$ and $H^{\tau}(\mathbb{S}) \hookrightarrow \mathrm{C}^{\tau-1 / 2}(\mathbb{S})$, it holds

$$
\|B\|_{\infty} \leq C\|\bar{\omega}\|_{H^{\tau}(\mathbb{S})} \prod_{i=1}^{n}\left\|b_{i}\right\|_{H^{r}(\mathbb{S})} \int_{-\pi}^{\pi}|s|^{\tau-3 / 2} \mathrm{~d} s \leq C\|\bar{\omega}\|_{H^{\tau}(\mathbb{S})} \prod_{i=1}^{n}\left\|b_{i}\right\|_{H^{r}(\mathbb{S})},
$$

and we are left with the function $A$. Taking advantage of the embedding $H^{r}(\mathbb{S}) \hookrightarrow \mathrm{C}^{r-1 / 2}(\mathbb{S})$, the arguments in the proof of [49, Lemma 3.1] show that indeed

$$
\|A\|_{\infty} \leq C \prod_{i=1}^{n}\left\|b_{i}\right\|_{H^{r}(\mathbb{S})}
$$

The estimates (A.15), (A.16) lead us to the estimate (A.3). The last two claims follow directly from (A.3) and the proof is complete.

\section{References}

[1] Amann, H.: Quasilinear evolution equations and parabolic systems. Trans. Am. Math. Soc. 293, 191-227 (1986)

[2] Amann, H.: Quasilinear parabolic systems under nonlinear boundary conditions. Arch. Ration. Mech. Anal. 92, 153-192 (1986)

[3] Amann, H.: Dynamic theory of quasilinear parabolic equations. I. Abstract evolution equations. Nonlinear Anal. 12, 895-919 (1988)

[4] Amann, H.: Nonhomogeneous linear and quasilinear elliptic and parabolic boundary value problems. In: Function Spaces, Differential Operators and Nonlinear Analysis (Friedrichroda, 1992), vol. 133 of Teubner-Texte Math., pp. 9126. Teubner, Stuttgart (1993)

[5] Amann, H.: Linear and Quasilinear Parabolic Problems. Vol. I, vol. 89 of Monographs in Mathematics, Birkhäuser Boston, Inc., Boston (1995) (Abstract linear theory)

[6] Ambrose, D.M.: Well-posedness of two-phase Hele-Shaw flow without surface tension. Eur. J. Appl. Math. 15, 597-607 (2004)

[7] Ambrose, D.M.: The zero surface tension limit of two-dimensional interfacial Darcy flow. J. Math. Fluid Mech. 16, 105-143 (2014)

[8] Angenent, S.B.: Nonlinear analytic semiflows. Proc. R. Soc. Edinb. Sect. A 115, 91-107 (1990)

[9] Bazaliy, B.V., Vasylyeva, N.: The two-phase Hele-Shaw problem with a nonregular initial interface and without surface tension. Zh. Mat. Fiz. Anal. Geom. 10, 3-43 (2014). 152, 155

[10] Bear, J.: Dynamics of Fluids in Porous Media. Dover Publications, New York (1988)

[11] Berselli, L.C., Córdoba, D., Granero-Belinchón, R.: Local solvability and turning for the inhomogeneous Muskat problem. Interfaces Free Bound. 16, 175-213 (2014)

[12] Castro, A., Córdoba, D., Fefferman, C., Gancedo, F.: Breakdown of smoothness for the Muskat problem. Arch. Ration. Mech. Anal. 208, 805-909 (2013)

[13] Castro, A., Córdoba, D., Fefferman, C.L., Gancedo, F., López-Fernández, M.: Turning waves and breakdown for incompressible flows. Proc. Natl. Acad. Sci. USA 108, 4754-4759 (2011)

[14] Castro, A., Córdoba, D., Fefferman, C.L., Gancedo, F., López-Fernández, M.: Rayleigh-Taylor breakdown for the Muskat problem with applications to water waves. Ann. Math. (2) 175, 909-948 (2012)

[15] Cheng, C.H.A., Granero-Belinchón, R., Shkoller, S.: Well-posedness of the Muskat problem with $H^{2}$ initial data. Adv. Math. 286, 32-104 (2016)

[16] Constantin, P., Córdoba, D., Gancedo, F., Rodrǵuez-Piazza, L., Strain, R.M.: On the Muskat problem: global in time results in 2D and 3D. Am. J. Math. 138, 1455-1494 (2016)

[17] Constantin, P., Córdoba, D., Gancedo, F., Strain, R.M.: On the global existence for the Muskat problem. J. Eur. Math. Soc. (JEMS) 15, 201-227 (2013)

[18] Constantin, P., Gancedo, F., Shvydkoy, R., Vicol, V.: Global regularity for 2D Muskat equations with finite slope. Ann. Inst. H. Poincaré Anal. Non Linéaire 34, 1041-1074 (2017)

[19] Crandall, M.G., Rabinowitz, P.H.: Bifurcation from simple eigenvalues. J. Funct. Anal. 8, 321-340 (1971)

[20] Crandall, M.G., Rabinowitz, P.H.: Bifurcation, perturbation of simple eigenvalues and linearized stability. Arch. Ration. Mech. Anal. 52, 161-180 (1973)

[21] Córdoba, A., Córdoba, D., Gancedo, F.: Interface evolution: the Hele-Shaw and Muskat problems. Ann. Math. (2) 173, 477-542 (2011)

[22] Córdoba, A., Córdoba, D., Gancedo, F.: Porous media: the Muskat problem in three dimensions. Anal. PDE 6, 447-497 (2013)

[23] Córdoba, D., Gancedo, F.: Contour dynamics of incompressible 3-D fluids in a porous medium with different densities. Commun. Math. Phys. 273, 445-471 (2007)

[24] Córdoba, D., Gancedo, F.: Absence of squirt singularities for the multi-phase Muskat problem. Commun. Math. Phys. 299, 561-575 (2010) 
[25] Córdoba,D., Lazar, O.: Global Well-Posedness for the 2D Stable Muskat Problem in $H^{3 / 2}$ (2018). arXiv:1803.07528

[26] Da Prato, G., Grisvard, P.: Equations d'évolution abstraites non linéaires de type parabolique. Ann. Mat. Pura Appl. (4) 120, 329-396 (1979)

[27] Deng, F., Lei, Z., Lin, F.: On the two-dimensional Muskat problem with monotone large initial data. Commun. Pure Appl. Math. LXX, 1115-1145 (2017)

[28] Ehrnström, M., Escher, J., Matioc, B.-V.: Steady-state fingering patterns for a periodic Muskat problem. Methods Appl. Anal. 20, 33-46 (2013)

[29] Escher, J.: The Dirichlet-Neumann operator on continuous functions. Ann. Scuola Norm. Sup. Pisa Cl. Sci. (4) 21, 235-266 (1994)

[30] Escher, J., Matioc, A.-V., Matioc, B.-V.: A generalized Rayleigh-Taylor condition for the Muskat problem. Nonlinearity 25, 73-92 (2012)

[31] Escher, J., Matioc, B.-V.: On the parabolicity of the Muskat problem: well-posedness, fingering, and stability results. Z. Anal. Anwend. 30, 193-218 (2011)

[32] Escher, J., Matioc, B.-V., Walker, C.: The domain of parabolicity for the Muskat problem. Indiana Univ. Math. J. 67, 679-737 (2018)

[33] Escher, J., Simonett, G.: Maximal regularity for a free boundary problem. NoDEA Nonlinear Differ. Equ. Appl. 2, 463-510 (1995)

[34] Escher, J., Simonett, G.: Analyticity of the interface in a free boundary problem. Math. Ann. 305, 439-459 (1996)

[35] Escher, J., Simonett, G.: Classical solutions of multidimensional Hele-Shaw models. SIAM J. Math. Anal. 28, 1028-1047 (1997)

[36] Friedman, A., Tao, Y.: Nonlinear stability of the Muskat problem with capillary pressure at the free boundary. Nonlinear Anal. 53, 45-80 (2003)

[37] Gancedo, F., García-Juárez, E., Patel, N., Strain, R.M.: On the Muskat problem with viscosity jump: global in time results. Adv. Math. 345, 552-597 (2019)

[38] Gancedo, F., Strain, R.M.: Absence of splash singularities for surface quasi-geostrophic sharp fronts and the Muskat problem. Proc. Natl. Acad. Sci. USA 111, 635-639 (2014)

[39] Gazolaz, D Córdoba, Granero-Belinchón, R., Orive-Illera, R.: The confined Muskat problem: differences with the deep water regime. Commun. Math. Sci. 12, 423-455 (2014)

[40] Granero-Belinchón, R., Shkoller, S.: Well-posedness and decay to equilibrium for the Muskat problem with discontinuous permeability. Trans. Am. Math. Soc. 372, 2255-2286 (2019)

[41] Granero-Belinchón, R.: Global existence for the confined Muskat problem. SIAM J. Math. Anal. 46, 1651-1680 (2014)

[42] Gómez-Serrano, J., Granero-Belinchón, R.: On turning waves for the inhomogeneous Muskat problem: a computerassisted proof. Nonlinearity 27, 1471-1498 (2014)

[43] Hong, J., Tao, Y., Yi, F.: Muskat problem with surface tension. J. Partial Differ. Equ. 10, 213-231 (1997)

[44] Kato, T.: Perturbation Theory for Linear Operators. Springer, Berlin (1995)

[45] Lu, J.K.: Boundary Value Problems for Analytic Functions. Series in Pure Mathematics, vol. 16. World Scientific Publishing Co. Inc., River Edge (1993)

[46] Lunardi, A.: Analytic Semigroups and Optimal Regularity in Parabolic Problems, Progress in Nonlinear Differential Equations and Their Applications, vol. 16. Birkhäuser, Basel (1995)

[47] Matioc, A.-V., Matioc, B.-V.: Well-posedness and stability results for a quasilinear periodic Muskat problem. J. Differ. Equ. 266, 5500-5531 (2019)

[48] Matioc, B.-V.: Viscous displacement in porous media: the Muskat problem in 2D. Trans. Am. Math. Soc. 370, 7511-7556 (2018)

[49] Matioc, B.-V.: The Muskat problem in two dimensions: equivalence of formulations, well-posedness, and regularity results. Anal. PDE 12, 281-332 (2019)

[50] Matioc, B.-V., Walker, C.: On the principle of linearized stability in interpolation spaces for quasilinear evolution equations. Monatshefte für Mathematik 191, 615-634 (2020)

[51] Murai, T.: Boundedness of singular integral operators of Calderón type. VI. Nagoya Math. J. 102, 127-133 (1986)

[52] Muskat, M.: Two fluid systems in porous media. The encroachment of water into an oil sand. Physics 5, 250-264 (1934)

[53] Patel, N., Strain, R.M.: Large time decay estimates for the Muskat equation. Commun. Partial Differ. Equ. 42, 977-999 (2017)

[54] Prüss, J., Simonett, G.: Moving Interfaces and Quasilinear Parabolic Evolution Equations, vol. 105 of Monographs in Mathematics. Springer, Cham (2016)

[55] Prüss, J., Simonett, G.: The Verigin problem with and without phase transition. Interfaces Free Bound. 20, 107-128 (2018)

[56] Prüss, J., Simonett, G., Wilke, M.: The Rayleigh-Taylor instability for the Verigin problem with and without phase transition. NoDEA Nonlinear Differ. Equ. Appl. 26, pp. Art. 18, 35 (2019)

[57] Prüss, J., Shao, Y., Simonett, G.: On the regularity of the interface of a thermodynamically consistent two-phase Stefan problem with surface tension. Interfaces Free Bound. 17, 555-600 (2015)

[58] Prüss, J., Simonett, G.: On the Muskat flow. Evol. Equ. Control Theory 5, 631-645 (2016)

[59] Saffman, P.G., Taylor, G.: The penetration of a fluid into a porous medium or Hele-Shaw cell containing a more viscous liquid. Proc. R. Soc. Lond. Ser. A 245, 312-329 (1958). (2 plates)

[60] Siegel, M., Caflisch, R.E., Howison, S.: Global existence, singular solutions, and ill-posedness for the Muskat problem. Commun. Pure Appl. Math. 57, 1374-1411 (2004) 
[61] Tofts, S.: On the existence of solutions to the Muskat problem with surface tension. J. Math. Fluid Mech. 19, 581-611 (2017)

[62] Yi, F.: Local classical solution of Muskat free boundary problem. J. Partial Differ. Equ. 9, $84-96$ (1996)

Bogdan-Vasile Matioc

Fakultät für Mathematik

Universität Regensburg

93040 Regensburg

Germany

e-mail: bogdan.matioc@ur.de

(accepted: March 26, 2020; published online: June 6, 2020) 